\title{
Jumps in option prices and their determinants: Real-time evidence from the E-mini S\&P 500 options market ${ }^{\S}$
}

\author{
George Kapetanios, ${ }^{a}$ Eirini Konstantinidi, \\ Michael Neumann, George Skiadopoulos ${ }^{d}$
}

\begin{abstract}
We provide first-time evidence of the real-time characteristics and drivers of jumps in option prices. To this end, we employ high-frequency data from the 24-hour E-mini S\&P 500 options market. We find that option prices do not jump simultaneously across strikes and maturities and are uncorrelated with jumps in the underlying futures price. We also find that $14 \%$ to $28 \%$ of detected option price jumps occur around scheduled news releases. However, it is illiquidity rather than the news content that drives these jumps. Evidence suggests that option traders increase bid-ask spreads to account for trading against investors who are skilled processors of public news releases. Interestingly, illiquidity does not drive jumps in the S\&P 500 index options market, where we also find sizable and idiosyncratic price jumps.
\end{abstract}

Keywords: Asymmetric information, co-jump, limit order book market, liquidity, option market, news announcement

JEL classification: C58, G10, G12, G13

\footnotetext{
$\S$ We are grateful to an anonymous referee and Amit Goyal (the Editor) for their constructive, stimulating, and thorough comments. We would also like to thank Richard Baillie, Alejandro Bernales, Menachem Brenner, Francis Breedon, Marcelo Fernandes, Robert Geske, Arie Gozluklu, Massimo Guidolin, Michel Habib, Erik Hjalmarsson, Andrew Karolyi, Arie Melnik, Dmitryi Muravyev, Anthony Neuberger, Neil Pearson, Tatjana Puhan, Olivier Scaillet, Ben Schreiber, Spyros Skouras, Theodoros Stamatiou, Zvi Wiener, Gal Zahavi, and participants at the Bank of England, Luxembourg School of Finance, University of Piraeus, University of Zurich seminar series, the 2013 MMF Workshop on Empirical Modelling of Financial Markets at Brunel University, the 2014 Jerusalem Finance Conference in honour of Professors Dan Galai and Itzhak Venezia at Hebrew University, the 2014 European Financial Management Conference (Rome), the 2014 Conference on Extreme Events in Finance at ESSEC Business School, and the 2018 Conference on Research on Economic Theory and Econometrics for useful discussions and comments. Financial support from the Chicago Mercantile Exchange Group Foundation and the University of Piraeus Research Centre is gratefully acknowledged.

${ }^{a}$ King's Business School, King's College London, UK, george.kapetanios@kcl.ac.uk

${ }^{b}$ Alliance Manchester Business School, University of Manchester, UK, eirini.konstantinidi@manchester.ac.uk ${ }^{c}$ Independent, michael.neumann.ln@gmail.com

${ }^{d}$ Corresponding author. School of Economics and Finance, Queen Mary, University of London, UK, and Department of Banking and Financial Management, University of Piraeus, Greece. Also Associate Research Fellow with Cass Business School and Warwick Business School, g.skiadopoulos@qmul.ac.uk, gskiado@unipi.gr
} 


\section{Introduction}

We provide first-time evidence on the characteristics and drivers of discontinuous changes, termed jumps, in option prices. We address four questions: Do jumps in option prices occur (1) simultaneously across strikes and maturities?, (2) as a result of jumps in the underlying asset market?, (3) as a result of news announcements?, and (4) as a result of shrinkages in liquidity?

These research questions are motivated by financial theory and are important to both academics and practitioners for three reasons. First, options have emerged as an important asset class and a number of studies examine their risk-return profile (Coval and Shumway, 2001; Driessen and Maenhout, 2007; Broadie et al., 2009; Santa-Clara and Saretto, 2009). Second, any option pricing model should generate the empirical characteristics of jumps to be consistent with the data. This is of particular importance in the context of option pricing models built to be consistent with the dynamics of market option prices (Jackwerth, 1999; Skiadopoulos, 2001). Third, the identification of option jump characteristics and determinants can shed light on the way that option prices are being formed in real-time.

To study the fine structure and real-time determinants of jumps, we employ high-frequency option quotes on the S\&P 500 E-mini futures options trading in a nearly 24-hour limit order book electronic market at the Chicago Mercantile Exchange (CME). We classify traded option contracts into 18 strike and time-to-maturity categories and compute 10-minute option returns for any given strike and maturity bucket. Then, we identify price jumps and their exact timings using Lee and Mykland's (2008) (LM, thereafter) jump detection test.

Next, we investigate the nature of detected option price jumps and their relation with three classes of determinants. First, we study whether option price jumps stem from jumps in the underlying asset's price and/or its volatility. Option pricing theory states that the dynamics 
of option prices are dictated by the dynamics of the price and volatility of the underlying asset. Second, we examine whether the occurrence, as well as the content of news releases is associated with jumps. The release of news is expected to trigger jumps in option prices via two channels: heterogeneous beliefs (Shefrin, 2001; Buraschi and Jiltsov, 2006; Friesen et al., 2012) and market sentiment (Han, 2008; Lemmon and Ni, 2011). We employ a set of U.S. scheduled macroeconomic news announcements, which are well monitored by academics and practitioners. ${ }^{1}$ The investigation of the real-time relation between jumps and scheduled news announcements is possible because our 24-hour dataset includes the times at which most scheduled U.S. macroeconomic news announcements are released. We also employ a comprehensive list of unscheduled news announcements; existing studies have paid little attention to how unscheduled news impacts asset prices. Third, we investigate whether the detected jumps in option prices may be due to changes in the liquidity of the option market. Christoffersen et al. (2018) find that option illiquidity predicts future option price increases.

We find that option prices jump. The probability of a jump occurring ranges from $0.22 \%$ to $0.56 \%$ depending on the option strike and maturity. Jumps are found to be negative on average, they are sizeable with an average size up to $63 \%$ of the option price, and they are mostly idiosyncratic (i.e., option prices in one strike and maturity category tend to jump independently from prices in other categories). This implies that the options market does not behave homogeneously in terms of the discontinuous movements of its prices. This finding is not surprising given that the S\&P 500 E-mini futures options market is populated by traders with different motives. Our finding also extends Sheikh and Ronn (1994), who provide evidence on the heterogeneity of the put and call option raw returns rather than the jump price dynamics trading in a limit order book market.

\footnotetext{
${ }^{1}$ We do not employ firm-specific announcements because the underlying asset in the employed options has to do with the aggregate market. The aggregate market will be affected by firm-specific news only to the extent that a firm has a dominant position in the market; there is no reason to expect that this is the case.
} 
We find that option price jumps are mostly unrelated to jumps in the underlying asset's price. These results complement the findings of Bakshi et al. (2000), who document that index call (put) prices do not always move in the same (opposite) direction with the underlying index and their dynamics differ across strikes and maturities. We document that $14 \%$ to $28 \%$ of the identified jumps occur around scheduled macroeconomic news releases depending on the strike and maturity. However, even though a fraction of jumps cluster around news announcements, we find that market illiquidity rather than the news content drives jumps in option prices. We also find that jumps unrelated to the release of scheduled news are also triggered by shrinking market liquidity. The shrinkage of market liquidity is manifested by an increase in options bid-ask spreads at the jump occurrence. These results are robust to the choice of the sample period (non-crisis vs. crisis periods) and to the choice of the set of news releases (scheduled vs. unscheduled news items).

Our findings on the relation between market liquidity and news-related jumps are consistent with the existence of informed trading in option markets (Chan et al., 1995; Easley et al., 1998; Chakravarty et al., 2004; Pan and Poteshman, 2006, and references therein). ${ }^{2}$ Option traders quote wider bid-ask spreads and thus they decrease market liquidity just before the macroeconomic news announcement to avoid trading with informed agents. This is consistent with Handa et al. (2003), who show that bid-ask spreads are a function of information asymmetry in a limit order book market. Moreover, our results extend the evidence in Erenburg and Lasser (2009), who find that in a limit order book market, the bid-ask spreads of index-linked securities increase around macroeconomic news releases.

We document that most of the news-related jumps are accompanied by zero trading vol-

\footnotetext{
${ }^{2}$ In the case of a dealers market, an increase in the option's bid-ask spread can also be attributed to the increase in inventory costs (Muravyev, 2016) and /or to the hedging costs of option market makers (Huh et al., 2014). However, the option market under consideration is not a dealers market since quotes can be provided by any type of investor; no information can be obtained on the type of investor who places orders.
} 
ume. This has two important implications. First, there are no informed option traders prior to scheduled macroeconomic announcements in the sense that there is no leakage of private information; if it were, then trading activity should take place prior to the announcement. This is inline with Ederington and Lee (1995), who find that there is no information leakage prior to scheduled news releases in the context of interest rate and foreign currency futures markets. Interestingly, this finding is in contrast to the evidence that there is private information prior to firm-specific announcements (Augustin et al., 2019 and references therein). Second, our finding on the relation between jumps and volume sheds light on the type of information asymmetry that traders are concerned about in option markets. Kim and Verrecchia $(1994 ; 1997)$ define two types of private information: private information that accrues to some investors due to leakage of information prior to an announcement and information that accrues to investors who are skilled in processing publicly announced information and thus effectively converting it to private. This is consistent with Kandel and Pearson (1995), who provide evidence that stock market participants interpret the same news release differently. Our findings suggest that option traders increase bid-ask spreads because they may also interact with investors who possess the latter type of private information.

We extend our analysis by considering S\&P 500 index options that are actively traded on the Chicago Board of Exchange (CBOE). We find that jumps are idiosyncratic just as in the E-mini options market. We also document that a fraction of the detected jumps is linked to scheduled macroeconomic news announcements; in $6 \%$ to $27 \%$ of the cases, a news announcement triggers a jump. Finally, we find that the option market illiquidity is not the key determinant of option jumps in this market.

Our paper contributes to four related strands of the literature. First, a number of studies find that a portion of jumps in asset prices are related to news announcements in the context 
of equities (Maheu and McCurdy, 2004; Rangel, 2011; Evans, 2011), bonds (Jiang et al., 2011), stock index futures, bond futures, and exchange rates (Lahaye et al., 2011). Jiang et al. (2011) and Boudt and Petitjean (2014) also find that changes in liquidity result in jumps in bond and equity prices, respectively.

Second, there is an extensive literature on real-time option price formation (e.g., Vijh, 1990; George and Longstaff, 1993; Sheikh and Ronn, 1994; Chan et al., 1995; Berkman, 1996; Chan et al., 2002; Chakravarty et al., 2004; Muravyev, 2016). However, these studies do not distinguish between continuous and discontinuous option price movements and they also focus on equity options. To the best of our knowledge, Taylor et al. (2013) is the only other study that explores the presence of jumps in option markets using high-frequency option prices; they consider options written on the FTSE 100. However, their scope differs from ours. They investigate which option pricing model can generate the detected option jumps, whereas we explore their economic sources.

Third, various studies examine the time evolution of the S\&P 500 implied volatilities (Skiadopoulos et al., 1999; Gonçalves and Guidolin, 2006; Neumann and Skiadopoulos, 2013). Again, these studies do not identify whether the observed changes in implied volatilities are smooth or discontinuous. Finally, previous studies explore the effect of news announcements on at-the-money equity options implied volatilities (Ederington and Lee, 1996; Fornari and Mele, 2001), as well as the option-implied VIX (Bailey et al., 2014). However, these studies do not investigate whether the impact of news releases creates discontinuities in option prices nor do they examine the entire spectrum of traded options individually. Most importantly, they explore the impact of news releases, whereas we take the reverse approach by detecting first jumps, and then we check their sources in the vicinity of their occurrence.

The remainder of this paper is organized as follows. In Section 2, we describe the E-mini 
S\&P 500 dataset and the way we structure it for our analysis. In Section 3, we introduce and apply Lee and Mykland's (2008) test to identify jumps in option prices across different strike and maturity categories. In Section 4, we investigate the determinants of option price jumps. We discuss a number of robustness checks in Section 5 and in Section 6 we consider the case of S\&P 500 index options. We conclude and outline the implications of our research in Section 7.

\section{Data}

\subsection{Option data}

We obtain intraday data for S\&P 500 E-mini futures options and the underlying futures (E-mini hereafter) from CME DataMine spanning from January 1, 2005 to December 31, 2010. The dataset includes the best bid and ask quotes time-stamped down to the second, the sizes quoted at the best bid and ask prices, the trading volume, and the transaction prices. Both options and futures contracts trade in a nearly 24-hour electronic market termed GLOBEX. ${ }^{3}$ The use of this dataset is of utmost importance for the purposes of our study because it allows us to identify any real-time association of detected jumps with the scheduled U.S. news releases. This is because most scheduled macroeconomic news announcements in the U.S. are released at 8:30 a.m. Eastern Standard Time (EST) taking place outside of the trading hours of most organized equity derivative exchanges. However, a real-time analysis is required as news announcement effects have been found to be relatively short-lived [for a similar choice in the context of futures markets, see, for example, Andersen et al. (2007)]. We sample quotes from 8.00 a.m. to 3.45

\footnotetext{
3 "E-mini" contracts are sized at one-fifth of the value of the regular contracts, making them more accessible to traders with small margin accounts. They trade almost continuously for five days a week on an open electronic limit order book system (GLOBEX) that is accessible to off-floor traders, as well to a number of market makers. GLOBEX is an international, automated order entry and matching system, which has a network extending to 10 financial centers, including New York, Chicago, London, and Tokyo. Trading on GLOBEX starts on Sundays at 6:00 p.m. EST and ends on Fridays at 4:15 p.m. EST. On Mondays through Thursdays, trading stops at 4:15 p.m. EST and restarts at 4:30 p.m. EST. There is also a daily maintenance shutdown from 5:30 p.m. EST to 6:00 p.m. EST on Mondays through Thursdays.
} 
p.m. EST to span the occurrence of scheduled news announcements.

Two more points are in order regarding the choice of the dataset. First, in line with Birru and Figlewski (2012), we employ best bid and ask quotes rather than transaction prices because only rarely do we observe simultaneous transaction prices for a large number of different contracts. This problem becomes particularly pronounced for the further out-of-the-money options and for options with longer maturities and it precludes us from performing our analysis on transaction prices. On the other hand, the best option quotes are available at all points in time and are continuously updated whenever the state of the order book changes. Moreover, we confirm that our quotes are accompanied by a large size relative to the trading volume and hence, are informative. Chan et al. (2002) find that option quotes can be more informative than trades. We discuss this issue further in Section 3. Second, the chosen time period contains both the mid 2007-2010 crisis period, as well as the previous non-crisis one. Therefore, we can check whether the number, as well as the nature of jumps in the options market differs over turbulent and non-turbulent periods.

CME offers two kinds of American style E-mini options, which differ by their expiration months. Quarterly options expire in March, June, September, and December, whereas serial options expire in January, February, and April. The underlying E-mini futures trade on quarterly expiries. Quarterly options are written on the E-mini that expire on the same day as the option. Serial options are written on the futures contract that has a maturity nearest to the option contract's. We match intraday options quotes with the simultaneously recorded underlying futures quotes and we discard observations for which this matching is not possible to avoid problems arising from non-synchronous underlying and option quotes. We also discard in-the-money option quotes because these options are highly illiquid [see Neumann and Skiadopoulos (2013) for a similar approach]. 
We apply a number of filters to the quotes of any given contract so as to minimize the impact of microstructure noise, which is likely to contaminate the quotes data. In particular, we apply the Barndorff-Nielsen et al. (2009) filtering criteria commonly used in the market microstructure literature. First, we replace bid and ask quotes with identical time stamps by their median bid and ask quotes for this time stamp. Second, we discard quotes for which the bid-ask spread is negative. Third, we discard quotes for which the bid-ask spread is "excessively" wide. We remove a contract's quotes whose spreads are greater than 50 times the daily median spread. ${ }^{4}$ We also discard quotes that are likely to represent outliers with respect to the midpoint quote. To this end, at any point in time where there is a quote, we compute the difference between the time $t$ observation and the median midpoint of bid and ask quotes of the 25 observations preceding and 25 observations following the time $t$ observation. We then calculate the daily mean of these differences. For any given day, we discard the observations that deviate by more than 10 times from this daily mean.

Next, we group option contracts into buckets based on their strikes and maturities. This classification serves two purposes. First, it provides a sufficient number of observations for each strike and maturity; tracking prices for each option contract at high frequencies is not feasible because not all strikes are traded. Second, it allows us to investigate whether the characteristics of discontinuous option price movements differ across strikes and maturities. An "idiosyncratic"

\footnotetext{
${ }^{4}$ The choice of any filtering criterion involves a trade-off between the amount of noise and the valuable information to be removed. Admittedly, in the market microstructure literature, the choice of threshold values may be ad hoc when it comes to applying data filters. More stringent criteria may also be applied. For instance, an alternative choice could be to first remove all contracts that correspond to zero option trading volume during the day, and then apply the standard Barndorff-Nielsen et al. (2009) filters to the quotes of the remaining contracts; we would like to thank the referee for pointing this out. However, even the bid-ask quotes corresponding to options with zero trading volume may contain useful information (Hiraki and Skiadopoulos, 2019). In addition, we group options into various categories depending on how close or far-away from the money they are. The average option trading volume conditional on the occurrence of a jump is smaller for close to-the-money rather than far-away from the money options (see Table 4). This provides further evidence that the detected jumps are not driven by liquidity concerns once option liquidity is proxied by the option trading volume. If trading volume is the driver of detected jumps, then the number of close-to-the-money option related jumps should be higher compared to the one of away from-the-money options. However, we find that the reverse holds in general (see Table 3).
} 
behavior of the discontinuous movements of option prices may be expected given that trading different options serves different purposes, and hence they may enjoy a different clientèle across the spectrum of strikes and maturities. Sheikh and Ronn (1994) and Bakshi et al. (2000) document such an idiosyncratic pattern in the call and put option raw returns, yet without isolating the jump component. In fact, the S\&P 500 E-mini futures options market is currently populated by off-floor traders, as well as by a number of market makers. ${ }^{5}$

We follow Bollen and Whaley (2004) and group option quotes according to their Black (1976) deltas into deep out-of-the-money (DOTM), out-of-the-money (OTM), and at-the-money (ATM) puts and calls; Panel A of Table 1 reports this classification [see Christoffersen et al. (2018) for a similar approach]. The computation of option deltas requires estimates for the risk-free rate, the underlying volatility, and the simultaneously recorded underlying price. We assume risk-free rates to be constant through the trading day. We proxy them by the daily U.S. LIBOR rates with maturities of one week, one month, two months, and up to 12 months obtained from the website of the St. Louis Federal Reserve. Whenever rates with maturities different from the ones covered by the data are required, we linearly interpolate between the rates of the two available adjacent maturities. We use the Black (1976) model to back out the implied volatility for each quote, and use it as the volatility input to calculate an option's delta. ${ }^{6}$ Option prices, as well as underlying prices are taken to be the mid-point of the bid and ask quotes. In addition to the delta dimension, we also classify option quotes into short-term, medium-term, and long-term options according to their time to expiration; Panel B of Table

\footnotetext{
${ }^{5}$ Currently, six market makers operate in this market: Citadel Derivatives Trading LLC, Chicago Trading Company, Deutsche Bank Securities, Inc., Goldman Sachs, Timber Hill, and Wolverine Trading LLC.

${ }^{6}$ Black's (1976) model prices European style options. However, its use to calculate the deltas and implied volatilities of the American style E-mini options is unlikely to introduce any error. This is because the early exercise premium is negligible given that we use ATM and OTM options with time-to-maturity less than 100 days (Barone-Adesi and Whaley, 1987). Hence, there is no loss in accuracy from using the computationally less expensive Black (1976) model. Using Black (1976)'s model does not assume that this model prices the options accurately. The Black (1976) model merely serves to map option prices as a function of strikes space to option prices as a function of deltas.
} 
1 reports this classification. The delta and maturity classifications yield 18 distinct groups of option quotes, which provide a parsimonious and accurate description of the structure of traded options.

For each one of these groups, we compute a time series of high-frequency returns, where each return is measured over a period of length $\Delta t$. To this end, we divide each trading day into $n_{d}=\frac{T_{d}}{\Delta t}$ subsamples, where $T_{d}$ is the number of observations per day. Then, for each one of these subsamples, we select the option quote with the delta closest to the midpoint delta of the delta category under scrutiny. Based on this quote and the latest quote for the same contract (i.e., same strike price and expiration date) before the end of the subsequent interval, we compute the high-frequency log option return for the delta category under scrutiny. This approach ensures that we compute option returns from the same contract. Then, we repeat the same process over the following subintervals. The application of this procedure to each subinterval and delta/maturity bucket yields a series of high-frequency option returns for all $18 \mathrm{delta} /$ maturity categories.

The empirical implementation of this scheme requires a choice for the subinterval $\Delta t$. In our jump detection test we assume that $\Delta t$ is arbitrarily small. Hence, it is desirable to choose the subinterval as short as possible. However, the more granular the sampling frequency, the more the data are contaminated by microstructure noise, which can distort the subsequent jump detection. Hence, in line with Andersen et al. (2000), we employ volatility signature plots of high-frequency option returns to select the "optimal" subinterval length. Volatility signature plots depict realized volatility as a function of the sampling frequency. In the absence of microstructure noise, realized volatility, defined as the squared root of summed squared intraday returns, should be invariant to changes in the sampling frequency provided the data is sampled fine enough. Figure 1 shows the average daily realized volatility over our sample as a 
function of different subinterval lengths for the various delta levels for the short-term options. Volatility signature plots for the other two maturity buckets are in the Online Appendix. We can see that the realized volatilities diverge as the subinterval length approaches zero, and they start converging around the 10-minute mark. Hence, we choose a subinterval length $\Delta t=10$ minutes. This choice yields between 52,627 to 64,755 return observations depending on the delta-maturity bucket.

\subsection{News announcement data}

We consider a list of scheduled U.S. macroeconomic news announcements, which includes 11 news items. We obtain the exact timing of the releases and their corresponding survey forecasts from Bloomberg. On Fridays, Bloomberg surveys key market participants for their forecasts regarding the values of economic variables that will be released within the next week. The median of the survey is taken to be the forecast for the respective economic variable. Table 2 reports the announcement items and their timing. All scheduled announcements take place within our daily sampling interval from 8:00 a.m. EST to 3:45 p.m EST with most of them being released at 8:30 a.m. EST on a monthly basis. The only exception is the FOMC rate announcement on 8/10/2008, which occurred at 7:00 a.m. EST; we exclude this announcement because it took place outside of our defined trading day. In total, our sample contains 888 announcements and 751 days on which at least one scheduled announcement was released.

Following Balduzzi et al. (2001), we consider news surprises to assess the impact of news announcements on option markets; in an efficient market, prices should not respond to information that has already been anticipated by market practitioners. Let $A_{i, t}$ denote the $i^{\text {th }}$ news item's actual figure released at time $t$ and let $F_{i, t}$ denote the forecast for this figure. Then, the surprise measure $S U R_{i, t}$ is defined as: 


$$
S U R_{i, t}:=\frac{A_{i, t}-F_{i, t}}{\sigma_{i}}
$$

where $\sigma_{i}$ denotes the sample standard deviation of the surprise components $A_{i, t}-F_{i, t}$ for the $i^{\text {th }}$ news item. We standardize the news surprises to facilitate comparison across different news items. As news surprises measure the component of a news release, which is unanticipated by the market, we will also refer to them as information shocks below.

\subsection{Liquidity measures}

Market liquidity is defined as the ability to buy or sell significant quantities of securities quickly at a low cost with little price impact. We compute two liquidity measures to proxy two important dimensions of the definition of market liquidity: the bid-ask spread and the option sizes ordered at the bid and ask prices. The bid-ask spread measures the cost of executing a trade for a given size, whereas the size variable measures the depth of the market (i.e., how many contracts are offered) at the best bid and ask price.

First, for each option delta and maturity category, we compute the time $t$ standardized bid-ask spread $s B A_{t}$ :

$$
s B A_{t}=\frac{A s k_{t}-B i d_{t}}{\sigma(B A)}
$$

where $A s k_{t}\left(B_{i d_{t}}\right)$ denotes the bid (ask) quote of the contract used to compute the 10-minute option returns and $\sigma(B A)$, the sample standard deviation of the dollar bid-ask spread $B A$ of the respective delta and maturity category. We compute a standardized bid-ask spread because the bid-ask magnitude depends on the option's strike and maturity.

Second, we obtain the time $t$ quoted sizes $\left(\right.$ AskSize $\left._{t}\right)$ and $\left(\right.$ BidSize $\left._{t}\right)$ at the best ask and bid quotes, respectively, for each delta/maturity category. To this end, we retain separately 
the ask and bid sizes of each one of the quotes used to compute the 10-minute option returns.

\section{$3 \quad$ Jumps in option prices}

\section{$3.1 \quad$ Jump test}

We employ Lee and Mykland's (LM, 2008) jump detection test to test whether there are any jumps in option prices. Compared to competing approaches, the LM test has the advantage that it detects both the occurrence and the timing of jumps [see Dumitru and Urga (2012) for a review of jump detection tests]. It does so by checking each recorded change in the asset price to determine whether or not this is a jump. It relies on the idea that large movements in an asset price can either be caused by jumps or they could be realizations of a continuous yet highly volatile process. Hence, it adjusts the observed movements by the volatility of the continuous part of the stochastic price process. If a given adjusted movement is "too large", then this change is labeled a jump.

Let $S(t)$ denote the time $t$ asset price. In the absence of jumps, the stochastic evolution of $S(t)$ is represented by:

$$
d \log S(t)=\mu(t) d t+\sigma(t) d W(t)
$$

where $W(t)$ is a Brownian motion. $\mu(t)$ and $\sigma(t)$ are the drift and volatility stochastic processes, respectively, such that $d \log S(t)$ is an Itô process with continuous sample paths. In contrast, if jumps are present, $S(t)$ is assumed to follow:

$$
d \log S(t)=\mu(t) d t+\sigma(t) d W(t)+Y(t) d J(t)
$$

where $J(t)$ denotes a counting process that controls the arrival of jumps and $Y(t)$ denotes the 
jump size.

Assume there are $n$ (equidistant) observations of $S(t)$ available and $t \in[0, T]$, where $T$ denotes the total number of observations of any given time series of option returns. Then, the distance between observations $\Delta t$ is given by $\Delta t=\frac{T}{n}$. We test whether there is a jump at a particular time $t_{i} \in[0, T]$. The LM test standardizes the log-return from $t_{i-1}$ to $t_{i}$ by the instantaneous volatility of the stochastic price process to account for its diffusive component. Thus, the LM test statistic is:

$$
\mathcal{L}(i) \equiv \frac{\log S\left(t_{i}\right)-\log S\left(t_{i-1}\right)}{\widehat{\sigma\left(t_{i}\right)}}
$$

where $\widehat{\sigma\left(t_{i}\right)}$ is estimated by the realized bipower variation (RBPV) using the past $K$ observations of $S(t)$. The RBPV estimator is given by:

$$
\left.{\widehat{\sigma\left(t_{i}\right)}}^{2} \equiv \frac{1}{K-2} \sum_{j=i-K+2}^{i-1} \mid \log S\left(t_{j}\right)-\log S\left(t_{j-1}\right)\right]|| \log \left[S\left(t_{j-1}\right)\right]-\log \left[S\left(t_{j-2}\right)\right] \mid
$$

RBPV estimates the instantaneous volatility consistently even in the presence of jumps in the past $K$ observations. LM show that under the null of no jumps and as $\Delta t \rightarrow 0$, the distribution of $\mathcal{L}(i)$ approximately follows the distribution of a normally distributed random variable with mean 0 and variance $\frac{1}{c^{2}}$ with $c=\sqrt{2} / \sqrt{\pi}$. In contrast, LM show that in the presence of jumps as $\Delta t \rightarrow 0, \mathcal{L}(i)$ becomes very large. Hence, observing large values of the test statistic inidicates the presence of jumps.

To assess how big the test statistic must be to indicate the presence of a jump at a certain significance level, LM employ the distribution of the maximum of the test statistic over $\mathcal{L}(i)$ under the null of no jumps. If the test statistic is greater than its maximum under the null of no jumps, it is highly unlikely that the observation in question was generated by a continuous 
process. As a consequence, one can base rejection of the null of no jumps on the rescaled and centered test statistic:

$$
\frac{|\mathcal{L}(i)|-C_{n}}{S_{n}}
$$

where $C_{n}=\frac{(2 \log (n))^{1 / 2}}{c}-\frac{\log (\pi)+\log (\log (n))}{2 c(2 \log (n))^{1 / 2}}, S_{n}=\frac{1}{c(2 \log (n))^{1 / 2}}$, and $n$ is the sample size. The null hypothesis of no jumps is rejected whenever $\frac{|\mathcal{L}(i)|-C_{n}}{S_{n}}$ exceeds the critical value $\beta^{*}$ obtained from a standard Gumbel cumulative distribution for a given confidence level $\alpha$ with $\beta^{*}$ such that $\exp \left(-\exp ^{-\beta^{*}}\right)=1-\alpha$, i.e., $\beta^{*}=-\log (-\log (1-\alpha))$

To implement the LM test, one has to select a window size $K$ for the purpose of estimating instantaneous volatility. In line with LM, we choose $K$ to be the smallest integer in the interval between $\sqrt{252 \times n o b s}$ and $252 \times$ nobs, where nobs denotes the number of observations per day. We determine the critical values by setting the Gumbel cumulative distribution function to a confidence level $\alpha=0.1 \%$. We choose such a conservative significance level to minimize the number of spuriously detected jumps; under the null hypothesis that there is no jump in any given subinterval, we expect to find a spuriously detected number of jumps equal to the number of observations times the chosen significance level. ${ }^{7}$

\subsection{Results}

We separately apply the LM test to the time series of the futures returns, and the option returns across the various delta and maturity categories. Table 3 reports the summary statistics (number of jumps, probability of a jump to occur, number of jump days, probability of a jump day to occur, average jump size, and percentage of negative jumps as a fraction of total jumps)

\footnotetext{
${ }^{7}$ We checked the robustness of the results with respect to changes in the significance level. We find that the results are qualitatively the same for different choices of the significance level ranging from $0.1 \%$ to $10 \%$.
} 
for each one of the delta and maturity categories. ${ }^{8}$ It also reports the same summary statistics for the underlying futures.

In Table 3, we can see that option prices jump. The number of jumps varies substantially across the delta and maturity buckets. With respect to the delta dimension, the DOTM calls and puts exhibit the greatest number of price jumps. With respect to the options maturity dimension, short maturity options exhibit more jumps than the longer maturity ones for all delta levels but OTM and DOTM calls. The documented heterogeneity of option jumps across moneyness and maturities is consistent with the fact that a number of traders with different motives trade in this market. Regarding the option's price jump size, this is negative on average and large (e.g., up to a $63 \%$ jump) with short-term options exhibiting substantially larger jump sizes than longer-term ones. The findings also suggest that downward option price jumps occur more often than upward jumps for almost all moneyness levels and maturities. Exact binomial tests reveal that the probability of observing a negative jump is significantly greater than $50 \%$ at the $5 \%$ significance level for most delta and maturity categories.

Finally, we compare the number of identified jumps in the price of the underlying asset to the number of identified option price jumps. We can see that the number of identified jumps in the underlying's price remains fairly constant across maturities; this is in contrast to the option price jump case. Furthermore, in most cases, the number of underlying jumps is less than the number of option price jumps. This finding has implications with respect to the question of how jumps are transmitted to option prices. It indicates that option price jumps cannot be solely attributed to simultaneous jumps in the price of the underlying asset. We investigate this relation further in the next section.

\footnotetext{
${ }^{8}$ Jump sizes are defined to be the realized returns that have been identified as a jump. Note that strictly defined, these returns are the sum of the drift, diffusive, and jump component. Measuring the exact jump size would require disentangling the drift and diffusive component from the realized return. This is beyond the scope of this paper.
} 
Two remarks are in order at this point regarding the credibility of our results. First, the employed best bid and ask quotes are reliable because they are associated with much larger sizes than the typical trading volume. Table 4 provides evidence for this. It shows the unconditional average size available at the best bid and ask price, as well as conditional on detecting a jump for the short-, medium-, and long-term options (Panels A, B, and C, respectively). Additionally, the average trading volume per 10-minute interval is reported. On average, the best bid and ask sizes are much greater than the typical 10-minute trading volume. Therefore, the bid and ask quotes are on average able to accommodate the typical 10-minute trading activity. Second, the LM statistic is a conservative test in the sense that it captures large jumps. This is ensured by the construction of the test statistic, as well as by the low significance level we employ. Therefore, detected jumps are unlikely to be a manifestation of noise.

\section{Drivers of option price jumps}

\subsection{Jumps in the underlying factors}

According to pricing theory, option prices are determined by the price of the underlying asset and its volatility by a no-arbitrage argument. In this subsection, we explore whether the detected jumps in option prices are due to jumps in the price of the underlying asset and/or its volatility. We define co-jumps (or simultaneous jumps) as the jumps which occur within the same 10-minute interval. If jumps in option prices arise from jumps in the underlying asset price and volatility, then one would expect these underlying factors to jump simultaneously with the prices of the ATM options (co-jumps); ATM options have the greatest (absolute) deltas and vegas. In addition, co-jumps across strikes may be observed. In the case where the underlying and or volatility co-jumps with DOTM options, options closer to at-the-money should jump as 
well because their deltas and vegas are greater than the DOTM options' ones.

To identify whether option prices co-jump, Figure 2 reports the frequency of different co-jump events for the short-, medium-, and long-term maturity buckets (Panels A, B, and C, respectively). A co-jump event is characterized by the number of concurrent jumps (i.e., jumps within the same 10-minute interval) across option prices of different delta levels and the underlying price. The panels depict for each maturity bucket how often option prices of one, two, three,..., six delta categories and/or the underlying price have jumped simultaneously. In particular, the case where the number of concurrent jumps is one refers to an idiosyncratic option price jump in one of the delta categories or in the underlying price. We can see that cojumps are rare. The vast majority of option price jumps are not accompanied by simultaneous jumps in the option prices of other delta buckets or by a simultaneous jump in the underlying price. $^{9}$

Yet, the mere evidence that most option price jumps are idiosyncratic across delta categieries does not rule out the possibility that some of the detected option price co-jumps are still due to price and/or volatility jumps. For instance, a jump in the underlying price may yield a jump in the ATM option price but not necessarily a jump in OTM and DOTM option prices. Similarly, cases where only ATM call and put prices jump simultaneously might be attributed to volatility jumps as these delta categories are more sensitive to changes in volatility than OTM/DOTM option prices. To investigate this further, we examine which option prices of delta categories and/or the underlying price jump simultaneously (termed composition of co-jump events).

Figure 3 shows the composition of co-jumps for the short-, medium-, and long-term maturi-

\footnotetext{
${ }^{9}$ We have also broadened the definition of co-jumps in two ways. First, we consider 10-minute intervals adjacent to the detected jump. Focusing on each maturity and delta bucket separately, we calculate the number of jumps that the remaining categories exhibit 10 minutes before, simultaneously, and 10 minutes after we detect a jump in the bucket under consideration. We confirm that the detected jumps are predominantly idiosyncratic. In the vast majority of the cases when we detect a jump in a specific bucket at time $t=0$, we do not detect any jumps 10 minutes before $(t=-10)$, simultaneously $(t=0)$ or 10 minutes after $(t=+10)$ in the remaining categories. This corroborates further that the detected jumps are predominantly idiosyncratic. These results are reported and discussed in the Online Appendix.
} 
ties (Panels A, B, and C, respectively). We can see that the already small number of detected co-jumps is spread across various delta and delta/underlying combinations; they do not show up in the ITM options and underlying category or in the ATM put and call options, which would be evidence for options co-jumping with the underlying factors. In addition, there is no specific pattern of the composition of co-jumps across maturities. Therefore, co-jumps do not cluster in a particular combination of delta categories.

To sum up, our findings suggest that option price jumps are not due to jumps in the underlying price and its volatility. Moreover, the presence of idiosyncratic jumps in option prices implies that there is not a common factor that explains the variability of the cross-section of jump-induced option returns. This is not at odds with studies that find that there are common factors in the cross-section of total (defined to be the sum of diffusive and discontinuous) option returns (e.g., Christoffersen et al., 2018).

\subsection{Information events as drivers of option price jumps}

We explore further the drivers of jumps in option prices. From a theoretical perspective, news announcements can make option prices jump. This is because heterogeneous beliefs (Shefrin, 2001; Buraschi and Jiltsov, 2006; Friesen et al., 2012) and market sentiment (Han, 2008; Lemmon and $\mathrm{Ni}, 2011$ ) are related to the slope of the implied volatility curve. ${ }^{10}$ Given that certain news may affect these factors drastically, one might expect these news effects to be transmitted to the slope of the implied volatility skew in a jump-like fashion. This will be manifested as jumps in option prices. Motivated by these considerations, we investigate whether jumps in option prices can also be related to macroeconomic news announcements.

To investigate to what extent detected option price jumps are related to scheduled macroe-

\footnotetext{
${ }^{10}$ At any given point in time and for any given option expiry, the implied volatility curve is defined to be the relation between the options implied volatilities and their respective strikes.
} 
conomic news announcements, we match the detected jumps with the the release of scheduled news announcements events (see Table 2). Here, an identified jump is related to a specific news announcement if the jump occurred within \pm 10 minutes of the respective announcement.

Panel A of Table 5 reports the conditional probabilities $P(N e w s \mid J u m p)$ and $P($ Jump $\mid$ News $)$ to detect the relation between the detected jumps and all considered macroeconomic news. $P($ News $\mid$ Jump $)$ shows the fraction of detected jumps associated with news announcements. $P($ Jump $\mid$ News $)$ shows the fraction of news associated with jumps (i.e., it denotes the probability that a news announcement triggers a jump).

Regarding $P($ News $\mid$ Jump), we can see that $14.35 \%$ to $28.50 \%$ of detected jumps are linked to the scheduled release of macroeconomic news depending on the delta and maturity category. The number of news-related jumps in the underlying asset differs substantially from the options ones. This indicates that the previously documented segmentation of option and underlying price jumps prevails around scheduled news announcements as well. Regarding $P($ Jump $\mid$ News $)$, the probability of news announcements yielding jumps is greater for DOTM calls and puts and it is greatest for short-term options; $P($ Jump $\mid$ News $)$ ranges from $1.35 \%$ to $6.86 \%$. Hence, it is more probable that a news announcement will yield an option price jump for shorter than for longer term options.

To provide more insight on the relative importance of the 11 individual news items shown in Table 2, we report the probabilities $P($ News $\mid$ Jump $)$ and $P(J u m p \mid N e w s)$ for each news item separately in Table 5, Panels B and C, respectively. Regarding $P(N e w s \mid$ Jump $)$, the nonfarm payrolls (NFP) report and the initial jobless claims (IJC) are associated with detected jumps more than the other releases. In particular, the NFP report is associated with up to $14.13 \%$ of the detected jumps, whereas IJC is associated up to $11.51 \%$ of the detected option price jumps. Regarding the probability $P(J u m p \mid N e w s)$ that a specific news release will trigger a jump, 
we can see that the NFP report is the news item that is most likely to trigger an option price jump, among all individual news items. For certain delta categories of short-term options, a NFP release results in a jump in more than $20 \%$ of all cases. This is in line with the literature on jumps and news announcements effects in financial markets that documents that the NFP report is the most influential scheduled news announcement (e.g., Andersen and Bollerslev, 1998). Interestingly, $P($ Jump $\mid$ News $)$ is masked when news announcements are aggregated; it increases from $1.4 \%$ to $7 \%$ when all news items are considered jointly to $20 \%$ when NFP is considered in isolation.

\subsection{Information shocks as sources of option price jumps}

Up to now our findings suggest that a fraction of option price jumps is triggered by news announcements. However, one may hypothesize that not only the fact that new information is released but also the content of the released information itself explains the occurrence of jumps.

We examine this hypothesis by statistically linking the occurrence of detected option price jumps to the content of the released scheduled macroeconomic news. To this end, we employ a logistic regression methodology [see Jiang et al. (2011) for a similar approach]. Lee (2012) shows that this approach allows drawing inference on the determinants of the unobservable stochastic jump intensity of the continuous time jump process even when one employs discrete time data (option returns and jump determinants). We estimate the following specification:

$$
P\left(\text { Jump }_{t} \mid \text { News }\right)=\frac{1}{1+\exp \left(-c-\sum_{j=1}^{11} \theta_{j}\left|S U R_{j, t}\right|\right)},
$$

where $j \in \quad\{$ NFP, CCI, CPI, DGO, FOMC, GDP, IJC, LI, NHS, PPI, RSA $\}$ and $P\left(J u m p_{t} \mid N e w s\right)$ denotes the probability that an option price jump will occur conditional on a scheduled macroeconomic announcement taking place; ex post it takes a value of 1 when 
there is a jump at the announcement time $t$, and 0 otherwise. This conditioning is necessary because the values of the macroeconomic surprises variables are only available at announcement times, which implies that equation (8) can only be estimated for observations coincident with announcement times. Therefore, a logistic regression unconditional on any news event cannot be conducted.

A few remarks are in order at this point regarding the estimation of equation (8). For any given delta and maturity category, the number of option price jumps that can be linked to the concurrent release of scheduled news is too small to estimate equation (8) accurately for each delta/maturity category separately. To increase the statistical accuracy of our estimates, we pool observations across different delta levels and estimate equation (8) once for each maturity category. We also only incorporate announcement items that exhibit at least one non-zero surprise matched with a concurrent option price jump. Pooling across different delta categories is not expected to affect our results for two reasons. First, the results of the analysis in Table 5 do not reveal any major differences across deltas with respect to the question of which news items are most important in explaining option price jumps. Second, we use absolute surprises and a binary jump indicator variable so that the expected sign of the $\theta_{j}$ is the same (positive) for all delta categories.

Panel A of Table 6 reports the estimation results for equation (8). In line with the evidence in Table 5, NFP surprises have a significant positive impact on the probability of a jump occuring in short- and medium-term options. There is no significant effect of NFP on the probability of a jump occurring in long-term options. For these options instead, GDP as well as retail sales less auto surprises have a positive impact on the jump probability. However, in general the evidence for a strong relation between news surprises and option price jumps appears to be rather weak; only a small number of coefficients in Panel A are significant. The 
results from the logistic regression approach suggest that the triggering of option price jumps by the considered news announcements is not due to the news content (i.e., due to the fact that new information is being impounded into prices). This implies that option price jumps are primarily driven by other determinants. We explore this further in the next subsection.

\subsection{Illiquidity as a driver of option price jumps}

As a final source of option price jumps, we investigate any effect of illiquidity on the associations between jumps and news releases. Christoffersen et al. (2018) find that option illiquidity reduces current option prices and predicts future option price increases. Hence, rapid movements in option liquidity might result in jumps in option prices.

First, we examine the effect of changes of liquidity on the probability of news-related option jumps. We re-estimate equation (8) by augmenting the set of covariates by the relative bid-ask spread, quoted size at the best bid price, and quoted size at the best ask price (see Subsection 2.3). We estimate:

$$
P\left(\text { Jump }_{t} \mid N e w s\right)=\frac{1}{1+\exp \left(-c-\sum_{j=1}^{11} \theta_{j}\left|S U R_{j, t}\right|-\sum_{k=1}^{3} \gamma_{k} I L_{k, t-1}\right)},
$$

where $I L_{k, t-1}$ denotes the time $t-1$ value of the $k^{t h}$ liquidity variable. We do not include variables related to the liquidity of the underlying futures market in equation (9). This is because the previous analysis showed that jumps in the option market are not related to jumps in the underlying market.

Panel B of Table 6 reports the estimation results for the model shown in equation (9). We can see that the coefficients of the standardized bid-ask spread are positive and highly significant for all maturity categories. The coefficients of the bid and ask sizes are negative, yet they are significant only sporadically. These results show that option price jumps are 
triggered by option market liquidity dry ups. Most importantly, almost all news surprise variables become insignificant after adding the liquidity variables to the model. Hence, after controlling for liquidity in the option market, the content of the considered news announcements has almost no power in explaining option price jumps. Similarly, Jiang et al. (2011) and Boudt and Petitjean (2014) find that illiquidity predicts jumps in bond and equity prices beyond information shocks induced by macroeconomic news announcements.

Our findings suggest that it is liquidity and not the content of the news surprises that drives the occurrence of option price jumps around announcements. To confirm this visually, for each option delta and maturity category, we compute the time $t$ relative bid-ask spread $B A_{t}$ :

$$
B A_{t}=\frac{A s k_{t}-B i d_{t}}{\left(A s k_{t}+B i d_{t}\right) / 2}
$$

where $A s k_{t}\left(B i d_{t}\right)$ denotes the bid (ask) quote of the contract used to compute the 10-minute option returns. We compute a relative bid-ask spread because the bid-ask magnitude depends on the option's strike and maturity [see Christoffersen et al. (2018) for a similar choice]. Figure 4 shows the median relative bid-ask spreads for a number of time subintervals around the news-related jumps (10 minutes before the jump up to 60 minutes after the jump) across the various moneyness levels for the case of the short maturity options. We can see that the spread increases significantly on the jump time (point zero in the graph) in the case of the short maturity options; the pattern is similar for the other two maturity buckets and it is not reported to conserve space.

The fact that the bid-ask spread increases on the announcement day can be explained by considering asymmetric information among traders as a key determinant of quoted bid-ask spreads in limit order book markets (Handa et al., 2003). Option traders widen the quoted bid-ask spreads and thus, they decrease market liquidity just before the news announcement 
to avoid the risk of trading with traders with superior information about the upcoming information event. This practice extends the evidence from index-linked limit order book markets (Erenburg and Lasser, 2009) and it is highly relevant to options markets because these are commonly viewed as a natural setting for informed traders (Chan et al., 1995; Easley et al., 1998; Chakravarty et al., 2004; Pan and Poteshman, 2006, and references therein).

To provide insight on the above information asymmetry explanation for the link between news-related jumps and liquidity, we investigate the nature of the information asymmetry. In the terminology of Kim and Verrecchia (1997), information asymmetry can be either "preannouncement" and / or "event-period" private information. The former stems from some information leakage that is not available to all traders. The latter type of information asymmetry stems from the fact that some traders have better skills in processing information when this is announced publicly, thus effectively making it private information (Kim and Verrecchia, 1994). In the case where there is no pre-announcement asymmetric information, there should be no relation between trading volume and price changes.

In Figure 5, we plot the distribution of traded volume on the jump time, as well as 10 minutes before the jump, to check whether option jumps are driven by pre-announcement private information. The figure shows the frequency (in \%) distribution of the option volume for any given delta bucket for the short-term options. We consider the percentage frequency for three volume buckets of zero, 10, and more than 10 contracts. Similar plots for medium- and long-term options are in the Online Appendix. We can see that the vast majority of news-related jumps is accompanied by zero volume. This indicates that there is no private information due to information leakage prior to scheduled news announcements [see Ederington and Lee (1995) for similar evidence]; if it were, then trading activity should take place prior to the announcement. Instead, traders increase their bid-ask spreads in the fear that they will interact with investors 
who are better skilled in processing information once the announcement is released; the increase in bid-ask spreads arises due to order cancellation or bid-ask spread revision. ${ }^{11}$

We confirm the absence of pre-announcement private information in the case of news-related jumps by re-estimating (9) by including the trading volume as an additional regressor:

$$
P\left(\text { Jump }_{t} \mid N e w s\right)=\frac{1}{1+\exp \left(-c-\sum_{j=1}^{11} \theta_{j}\left|S U R_{j, t}\right|-\sum_{k=1}^{3} \gamma_{k} I L_{k, t-1}-\beta V o l_{t-1, t}\right)},
$$

where $\operatorname{Vol}_{t-1, t}$ denotes the total trading volume in the respective delta/maturity bucket from time $t-1$ to $t$. Panel A of Table 7 reports the estimation results for the model shown in equation (11). We can see that the option trading volume variable is insignificant, as expected. This confirms that the observed increase in the bid-ask spreads of options is not due to preannouncement private information.

To robustify the evidence on the link between illiquidity and option price jumps, we explore the determinants of option price jumps that are not related to scheduled news announcement times. We estimate the following logistic regression based on a pooled (across delta categories) sample of non-news related observations:

$$
P\left(\text { Jump }_{t} \mid N_{o} \text { News }\right)=\frac{1}{1+\exp \left(-c-\sum_{k=1}^{3} \gamma_{k} I L_{k, t-1}-\beta V o l_{t-1, t}\right)} .
$$

Panel B of Table 7 reports the estimation results. We can see that non-news related jumps are strongly related to increases in illiquidity in option markets. This result holds for all liquidity measures considered. Our findings suggest that option market illiquidity is by far the most important driver of option price jumps. Interestingly, the option trading volume is significant

\footnotetext{
${ }^{11}$ The information asymmetry explanation for the detected jumps cannot not explain on its own the documented non-synchronous occurrence of jumps across strikes. This is because an informed trader may choose which strike to trade by taking other factors into account such as leverage, transaction costs, commissions etc. (Chakravarty et al., 2004, and references therein). The relative importance of these factors may also change through time, thus also partially explaining the idiosyncraticity of the detected jumps.
} 
now. The significance of the liquidity and volume variables reveals an explanation for the occurrence of the no-news related jumps. In this case, the increase in bid-ask spreads cannot be attributed to an information asymmetry story due to the nature of detected jumps. Instead, it is attributed to the fact that the increase in trading activity fills in orders and as a result the bid-ask quotes that are further down in the order book advance to the top of the order book.

\section{$5 \quad$ Further robustness analysis}

We provide further robustness tests. First, we conduct a subsample analysis. Second, we consider the relation of detected jumps with unscheduled news announcements. Third, we use a different index options dataset, namely data on S\&P cash-settled index options.

\subsection{Subsample analysis}

We investigate the existence of option price jumps over two subsamples. We divide the entire sample period from May 1, 2005 to December 31, 2010 into a non-crisis and a crisis period spanning May 1, 2005 to July 31, 2007 and August 1, 2007 to December 31, 2010, respectively; August 2007 is typically considered to mark the beginning of the global financial crisis. We then recompute the jump and jump-news statistics as in Table 5, separately, for both the non-crisis and crisis subsamples.

Panels A and B of Table 8 report summary statistics for the detected option price jumps for the non-crisis and crisis periods, respectively. We can see that the jump frequencies in both subsamples are of similar magnitude, for any given maturity category. Hence, jumps in option prices exist regardless of the general market conditions, contradicting conventional perception that jumps mainly occur during crisis periods.

Panels A and B of Table 9 report the summary statistics on the relation between jumps 
and all scheduled macroeconomic news announcements for the non-crisis and the crisis periods, respectively. The comparison of the non-crisis to the crisis figures reveals an interesting pattern. The association of jumps and news is stronger in the crisis subsample than in the non-crisis subsample. Both the probability of news to cause a jump as well as the fraction of jumps related to news announcements are substantially greater in the crisis sample than in the noncrisis subsample for almost all delta and maturity categories. In the most pronounced case (short-term DOTM calls), the probability of a news announcement triggering a jump is almost three times greater in the crisis sample than in the non-crisis subsample. This suggests that option markets have been more sensitive to the release of macroeconomic news announcements during the crisis period than in the non-crisis period.

Finally, Panels A and B (C and D) of Table 10 report $P($ News $\mid$ Jump) and $P($ Jump $\mid$ News $)$ disaggregated by news items for the non-crisis (crisis) period. We can see that the results are in line with the results aggregated over all announcements as well as with the results over the full sample (Subsection 4.2). The NFP report as well as the IJC turn out to be the news items most associated with jumps in both periods. Also, the jump-news relation appears to be stronger in the crisis period than in the non-crisis period.

The more pronounced clustering of option price jumps around the release of scheduled news in the crisis period also raises the question of whether the news content is more powerful in explaining the occurrence of option price jumps in the crisis period. In fact, the empirical evidence in the context of spot markets suggests that macroeconomic news surprises affect equity prices differently depending on the state of the business cycle (Andersen et al., 2007). This diverse response of jumps to news surprises across different states of the economy might also carry over to option markets.

We re-estimate the logistic regression equation (11) on the crisis subsample to provide 
insight on whether the news content is more powerful in explaining the occurrence of option price jumps in this period. Table 11 reports the estimation results. Surprisingly, even though we find the association between news events and option price jumps is stronger in the crisis period, the explanatory power of the content of news surprises for option price jumps turns out to be low. Only three news surprise coefficients turn out to be significant.

As a further robustness check, we examine the dynamics of option market illiquidity over the non-crisis and crisis periods. Given that we find that the likelihood of option price jumps is similar across the crisis and non-crisis subsamples, one would expect the dynamics of option market illiquidity not to differ either. This is because our results over the full sample period suggest that the arrival of option price jumps is mostly driven by the option market's illiquidity.

Figure 6 depicts the evolution of the daily average relative bid-ask spread for short-term options of the various delta categories. Similar plots for the remaining maturities are in the Online Appendix. We can see that the dynamics of option market illiquidity are comparable in the non-crisis and crisis periods. In particular, the dynamics of illiquidity do not appear to be any more erratic in the crisis subsample than in the non-crisis subsample. This is in line with our previous finding that option price jumps are equally likely in the non-crisis and crisis periods provided option price jumps are driven by option market illiquidity. Hence, we conclude that the results from the subsample analysis further confirm that it is liquidity and not the content of news shocks that drive jumps in option prices.

\subsection{Unscheduled news announcements}

As a second robustness check, we extend the set of information shocks considered in our analysis. The vast majority of studies examining news announcement effects on financial markets have focussed on the analysis of scheduled news announcements (Andersen et al., 2007; Lahaye et al., 
2011). Our list of scheduled macroeconomic news (see Table 2) includes the news items most commonly used in the literature and it can be regarded as a comprehensive list of the universe of scheduled information shocks. However, information shocks might also arise from the release of unscheduled news.

In what follows, we match detected option price jumps with a set of unscheduled news announcements. The list of announcements considered is taken from Jiang et al. (2012) and it includes 137 unscheduled announcement items. The selection of these items is based on the chronology of significant events of the California Department of Finance, the crisis time line provided by the Federal Reserve Bank of St. Louis, and the European crisis time line provided by Bloomberg.

Tracing the exact intraday timing of an unscheduled news announcement is not feasible because different data sources provide a different timing. Hence, we match detected option price jumps with unscheduled news announcements on a daily level. In particular, we define an unscheduled news day to be the day on which at least one unscheduled news announcement has been released and we compute the fraction of jump days that are equal to unscheduled news days. A remark is in order at this point. Seventy-two of the unscheduled news days in our sample coincide with scheduled news days as well. Consequently, it is not possible to unambiguously attribute a jump day to either unscheduled or scheduled news on these days. Therefore, we only retain unscheduled news days on which there has been no release of scheduled news.

Table 12 reports the results of the resulting jump day-unscheduled news day matching. We can see that unscheduled news play a minor role in explaining option price jump days. Only up to $6 \%$ of the detected option price jump days might be attributed to the release of unscheduled news across all delta/maturity categories. Hence, we can conclude that by 
focussing on our set of scheduled news announcements, we do not ignore any relevant effect arising from unscheduled news announcements. Furthermore, the unimportance of unscheduled news for explaining option price jumps is consistent with liquidity being the most important jump determinant. This is because unscheduled news announcements occur unexpectedly by definition and thus they cannot adversely affect market liquidity through the informed trading channel described in Subection 4.4 .

\section{The case of S\&P 500 index options}

We extend our analysis by considering S\&P 500 index options. S\&P 500 index options are actively traded on the CBOE from 9:30 a.m. to 4:15 p.m. EST over our entire sample period, i.e., at times beyond 8:30 a.m. EST, which is when most scheduled macroeconomic news announcements occur. The E-mini S\&P 500 options market that we examined in the previous sections, has the advantage that it is a 24-hour market and thus, we can match scheduled macroeconomic news announcements to the detected jumps. In the case of the S\&P 500 index options, we capture, even imperfectly, the effect of pre-market opening news announcements by considering overnight returns.

We obtain OPRA trade and quote data on the S\&P 500 index options from TickData for the period from January 1, 2005 to December 31, 2010. This data set includes bid and ask quotes, quote size, trade price, and trade size time-stamped at least down to the second. We sample quotes from 9:30 a.m. to 3:45 p.m. EST. We apply the same filters as the ones used in our main analysis to remove noisy data and minimize the effect of microstructure noise (BarndorffNielsen et al., 2009). Next, we group options into the maturity and delta buckets shown in Table 1. To allocate each intraday observation to a delta bucket, we estimate implied volatility and delta using the Black (1976) model. We consider the mid-quote of the shortest E-mini 
futures contract as the underlying asset price of S\&P 500 index options; this is different from the underlying asset price of E-mini options, where we use the mid-quote of the E-mini futures contract with the same or nearest maturity to the option contract. To avoid non-synchronicity concerns, we match the shortest E-mini S\&P 500 futures quotes to simultaneous S\&P 500 index option quotes. We also proxy intraday interest rates with the daily linearly interpolated U.S. LIBOR rates (LIBOR maturities between one week and one year).

Once we group our options data, we calculate 10-minute returns for the underlying shortest e-mini S\&P 500 futures contract and each one of the 18 options buckets. Given that the majority of the news announcements occurs at 8:30 a.m. EST, i.e., before the S\&P 500 index option market opens, we also calculate overnight returns, in addition to the over-day returns. We calculate overnight returns using quotes from the last 10-minute interval of the previous day and the first 10-minute interval of the current day. Subsequently, we apply the LM test to detect jumps.

Overall, we find that jumps are idiosyncratic in the sense that they do not jump simultaneously (i.e., with the same 10-minute interval), just as was the case with the E-mini options market. We also document that a fraction of the detected jumps is linked to scheduled macroeconomic news announcements; in $4.7 \%$ to $10.8 \%$ of the cases, a news announcement triggers a jump. Finally, we find that illiquidity is not the key determinant of option jumps. In fact, the content of news announcements (and not illiquidity variables) significantly affects the probability of a news-related S\&P 500 option price jumps. Moreover, the relative bid-ask spread is stable around news-related jumps. More information and results can be found in the Online Appendix. 


\section{Conclusions}

We provide first-time evidence on the characteristics and drivers of option price jumps by employing high-frequency index options quotes from a limit order book market. We find that E-mini S\&P 500 option price jumps are rare, sizeable, do not occur simultaneously across strikes and maturities, and are uncorrelated with jumps in the underlying futures price. On the other hand, only $14 \%$ to $28 \%$ of the detected option price jumps are associated with scheduled releases. However, even though news announcements trigger a fraction of option price jumps, the specific news content does not. Instead, we find that the option market's liquidity measured by option bid-ask spreads drives option price jumps. Moreover, we document that the increase in option bid-ask spreads is not explained by trading activity.

Our findings have three implications. First, jumps in E-mini S\&P 500 option quotes are idiosyncratic. This extends the findings of Sheikh and Ronn (1994) and Bakshi et al. (2000), who document that total (i.e., the sum of continuous and discontinuous) option returns exhibit heterogeneous dynamics across traded option contracts. Second, the E-mini S\&P 500 option market is segmented from the underlying market in terms of the discontinuous changes in asset prices. This complements the findings of Bakshi et al. (2000), who find that option prices do not always move in line with the underlying asset price. Third, the fact that liquidity rather than the content of information shocks drives E-mini S\&P 500 option price jumps may also be explained by the fact that illiquidity increases prior to news announcements so that traders protect themselves against informed traders. Our evidence also suggests that the informational advantage in this option market is not due to some private information as a result of information leakage prior to an announcement. Instead, the information asymmetry stems from the fact that some investors may be more skilled in processing the released information than others, thus making market makers increase bid-ask spreads; this increase is manifested as an option 
price jump. Interestingly, in the case of the S\&P 500 index options market, where we also find sizable and idiosyncratic price jumps, the content of news announcements (and not illiquidity) is the key determinant of option jumps.

Our analysis can be extended to other option markets to check whether our findings carry through there as well. It would also be worth exploring whether option pricing models proposed by previous studies can generate the documented jump patterns in option prices. If they do not, one should look into developing a limit order book market microstructure model that generates idiosyncratic jumps in the cross-section of option prices. Moreover, our results support the call for incorporating option market liquidity risk into option pricing theory (Christoffersen et al., 2018). Finally, our evidence is of interest to exchanges for the purposes of setting option margins. 


\section{References}

Andersen, T. G. and Bollerslev, T. (1998). Deutsche mark-dollar volatility: Intraday activity patterns, macroeconomic announcements, and longer run dependencies, Journal of Finance 53: 219-265.

Andersen, T. G., Bollerslev, T., Diebold, F. X. and Laby, P. (2000). Great realisations, Risk March 2000: 105-108.

Andersen, T. G., Bollerslev, T., Diebold, F. X. and Vega, C. (2007). Real-time price discovery in global stock, bond and foreign exchange markets, Journal of International Economics 73: 251-277.

Augustin, P., Brenner, M. and Subrahmanyam, M. G. (2019). Informed options trading prior to takeover announcements: Insider trading?, forthcoming in Management Science.

Bailey, W., Zheng, L. and Zhou, Y. (2014). What makes the VIX tick?, Working Paper, Cornell University .

Bakshi, G., Cao, C. and Chen, Z. (2000). Do call prices and the underlying stock always move in the same direction?, Review of Financial Studies 13: 549-584.

Balduzzi, P., Elton, E. J. and Green, T. C. (2001). Economic news and bond prices: Evidence from the U.S. treasury market, Journal of Financial and Quantitative Analysis 36: 523-543.

Barndorff-Nielsen, O. E., Hansen, P. R., Lunde, A. and Shephard, N. (2009). Realized kernels in practice: Trades and quotes, Econometrics Journal 12: C1-C32.

Barone-Adesi, G. and Whaley, R. E. (1987). Efficient analytical approximation of American option values, Journal of Finance 42: 301-320. 
Berkman, H. (1996). Large option trades, market makers, and limit orders, Review of Financial Studies 9: 977-1002.

Birru, J. and Figlewski, S. (2012). Anatomy of a meltdown: The risk neutral density for the S\&P 500 in the fall of 2008, Journal of Financial Markets 15: 151-180.

Black, F. (1976). The pricing of commodity contracts, Journal of Financial Economics 3: 167179.

Bollen, N. P. B. and Whaley, R. E. (2004). Does net buying pressure affect the shape of implied volatility functions?, Journal of Finance 59: 711-753.

Boudt, K. and Petitjean, M. (2014). Intraday liquidity dynamics and news releases around price jumps: Evidence from the djia stocks, Journal of Financial Markets 17: 121-149.

Broadie, M., Chernov, M. and Johannes, M. (2009). Understanding index option returns, Review of Financial Studies 22: 4493-4529.

Buraschi, A. and Jiltsov, A. (2006). Model uncertainty and option markets with heterogeneous beliefs, Journal of Finance 61: 2841-2897.

Chakravarty, S., Gulen, H. and Mayhew, S. (2004). Informed trading in stock and option markets, Journal of Finance 59: 1235-1257.

Chan, K., Chung, Y. P. and Fong, W. (2002). The informational role of stock and option volume, Review of Financial Studies 15: 1049-1075.

Chan, K., Chung, Y. P. and Johnson, H. (1995). The intraday behavior of bid-ask spreads for NYSE stocks and CBOE options, Journal of Financial and Quantitative Analysis 30: 329346. 
Christoffersen, P., Goyenko, R., Jacobs, K. and Karoui, M. (2018). Illiquidity premia in the equity options market, Review of Financial Studies 31: 811-851.

Coval, J. D. and Shumway, T. (2001). Expected option returns, Journal of Finance 56: 9831009.

Driessen, J. and Maenhout, P. (2007). An empirical portfolio perspective on option pricing anomalies, Review of Finance 11: 561-603.

Dumitru, A.-M. and Urga, G. (2012). Identifying jumps in financial assets: A comparison between nonparametric jump tests, Journal of Business and Economic Statistics 30: 242255.

Easley, D., O'Hara, M. and Srinivas, P. (1998). Option volume and stock prices: Evidence on where informed traders trade, Journal of Finance 53: 431-465.

Ederington, L. H. and Lee, J. H. (1995). The short-run dynamics of the price adjustment to new information, Journal of Financial and Quantitative Analysis 30: 117-134.

Ederington, L. H. and Lee, J. H. (1996). The creation and resolution of market uncertainty: The impact of information releases on implied volatility, Journal of Financial and Quantitative Analysis 31: 513-539.

Erenburg, G. and Lasser, D. (2009). Electronic limit order book and order submission choice around macroeconomic news, Review of Financial Economics 18: 172-182.

Evans, K. P. (2011). Intraday jumps and US macroeconomic news announcements, Journal of Banking and Finance 35: 2511-2527.

Fornari, F. and Mele, A. (2001). Volatility smiles and the information content of news, Applied Financial Economics 11: 179-186. 
Friesen, G. C., Zhang, Y. and Zorn, T. S. (2012). Heterogeneous beliefs and risk neutral skewness, Journal of Financial and Quantitative Analysis 47: 851-872.

George, T. J. and Longstaff, F. A. (1993). Bid-ask spreads and trading activity in the S\&P 100 index options market, Journal of Financial and Quantitative Analysis 28: 381-397.

Gonçalves, S. and Guidolin, M. (2006). Predictable dynamics in the S\&P 500 index options implied volatility surface, Journal of Business 79: 1591-1635.

Han, B. (2008). Investor sentiment and option prices, Review of Financial Studies 21: 387-414.

Handa, P., Schwartz, R. and Tiwari, A. (2003). Quote setting and price formation in an order driven market, Journal of Financial Markets 6: 461-489.

Hiraki, K. and Skiadopoulos, G. (2019). The contribution of frictions to expected returns, Working Paper, Queen Mary, University of London .

Huh, S.-W., Lin, H. and Mello, A. S. (2014). Hedging by option market makers: Theory and evidence, Working Paper, University of Wisconsin at Madison .

Jackwerth, J. C. (1999). Option implied risk-neutral distributions and implied binomial trees: A literature review, Journal of Derivatives 7: 66-82.

Jiang, G. J., Konstantinidi, E. and Skiadopoulos, G. (2012). Volatility spillovers and the effect of news announcements, Journal of Banking and Finance 36: 2260-2273.

Jiang, G. J., Lo, I. and Verdelhan, A. (2011). Information shocks, liquidity shocks, jumps, and price discovery: Evidence from the US treasury market, Journal of Financial and Quantitative Analysis 46: 527-551.

Kandel, E. and Pearson, Neil, D. (1995). Differential interpretation of public signals and trade in speculative markets, Journal of Political Economy 103: 831-872. 
Kim, O. and Verrecchia, R. E. (1994). Market liquidity and volume around earnings announcements, Journal of Accounting and Economics 17: 41-67.

Kim, O. and Verrecchia, R. E. (1997). Pre-announcement and event-period private information, Journal of Accounting and Economics 24: 395-419.

Lahaye, J., Laurent, S. and Neely, C. J. (2011). Jumps, cojumps and macro announcements, Journal of Applied Econometrics 26: 893-921.

Lee, S. S. (2012). Jumps and information flow in financial markets, Review of Financial Studies 25: 439-479.

Lee, S. S. and Mykland, P. A. (2008). Jumps in financial markets: A new nonparametric test and jump dynamics, Review of Financial Studies 21: 2535-2563.

Lemmon, M. L. and Ni, S. X. (2011). The effects of investor sentiment on speculative trading and prices of stock and index options, Working Paper, University of Utah .

Maheu, J. M. and McCurdy, T. H. (2004). News arrival, jump dynamics, and volatility components for individual stock returns, Journal of Finance 59: 755-793.

Muravyev, D. (2016). Order flow and expected option returns, Journal of Finance 71: 673-708.

Neumann, M. and Skiadopoulos, G. (2013). Predictable dynamics in higher order risk-neutral moments: Evidence from the S\&P 500 options, Journal of Financial and Quantitative Analysis 48: 947-977.

Pan, J. and Poteshman, A. (2006). The information in options volume for future stock prices, Review of Financial Studies 19: 871-908.

Rangel, J. G. (2011). Macroeconomic news, announcements, and stock market jump intensity dynamics, Journal of Banking and Finance 35: 1263-1276. 
Santa-Clara, P. and Saretto, A. (2009). Option strategies: Good deals and margin calls, Journal of Financial Markets 12: 391-417.

Shefrin, H. M. (2001). On kernels and sentiment, Working Paper, Santa Clara University .

Sheikh, A. M. and Ronn, E. I. (1994). A characterization of the daily and intraday behavior of returns on options, Journal of Finance 49: 557-579.

Skiadopoulos, G., Hodges, S. and Clewlow, L. (1999). The dynamics of the S\&P 500 implied volatility surface, Review of Derivatives Research 3: 263-282.

Skiadopoulos, G. S. (2001). Volatility smile consistent option models: A survey, International Journal of Theoretical and Applied Finance 4: 403-437.

Taylor, S. J., Tzeng, C.-F. and Widdicks, M. (2013). Information about price and volatility jumps inferred from option prices, Journal of Futures Markets 38: 1206-1226.

Vijh, A. M. (1990). Liquidity of the CBOE equity options, Journal of Finance 45: 1157-01179. 
Table 1: Option categories

\begin{tabular}{llc}
\hline Category & Name & $\begin{array}{c}\text { Delta interval/ } \\
\text { Time to expiration } T \text { (in days) }\end{array}$ \\
\hline Panel $\boldsymbol{A}$ : Delta categories & \\
1 & Deep out-of-the-money (DOTM) put & $-0.125<\Delta \leq-0.02$ \\
2 & Out-of-the-money (OTM) put & $-0.375<\Delta \leq-0.125$ \\
3 & At-the-money (ATM) put & $-0.625<\Delta \leq-0.375$ \\
4 & At-the-money (ATM) call & $0.375<\Delta \leq 0.625$ \\
5 & Out-of-the-money (OTM) call & $0.125<\Delta \leq 0.375$ \\
6 & Deep out-of-the-money (DOTM) call & $0.02<\Delta \leq 0.125$ \\
& & \\
Panel $\boldsymbol{B}:$ Maturity categories & \\
1 & Short-term options & $10 \leq T \leq 40$ \\
2 & Medium-term options & $40<T \leq 70$ \\
3 & Long-term options & $70<T \leq 100$ \\
\hline
\end{tabular}

Entries report the definition of the option delta categories in terms of their Black (1976) options delta (Panel A) and of the option expiration categories in terms of their number of days to expiration (Panel B). 


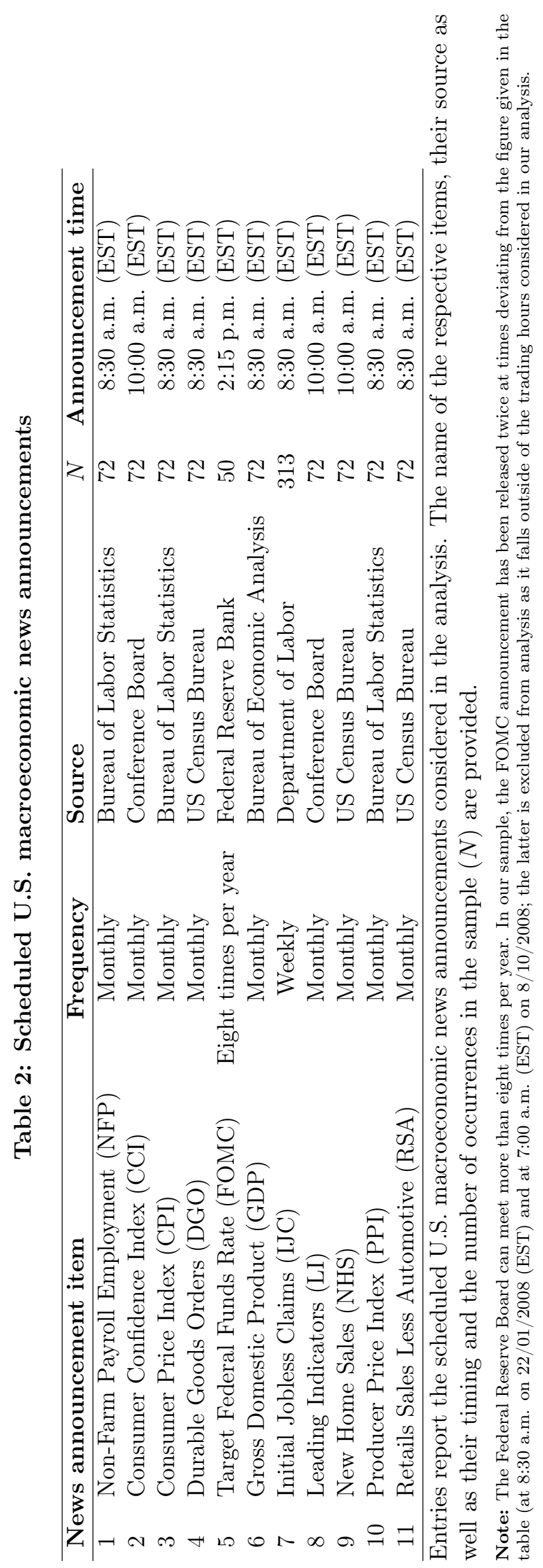


Table 3: Summary statistics of detected jumps

\begin{tabular}{|c|c|c|c|c|c|c|c|}
\hline & $\begin{array}{c}\text { DOTM } \\
\text { puts }\end{array}$ & $\begin{array}{l}\text { OTM } \\
\text { puts }\end{array}$ & $\begin{array}{c}\text { ATM } \\
\text { puts }\end{array}$ & $\begin{array}{c}\text { ATM } \\
\text { calls }\end{array}$ & $\begin{array}{l}\text { OTM } \\
\text { calls }\end{array}$ & $\begin{array}{c}\text { DOTM } \\
\text { calls }\end{array}$ & Futures \\
\hline \multicolumn{8}{|l|}{ Short-term options } \\
\hline \# Observations & 62,142 & 63,142 & 62,734 & 62,815 & 63,074 & 61,549 & 64,665 \\
\hline \# Days & 1,437 & 1,437 & 1,437 & 1,437 & 1,437 & 1,437 & 1437 \\
\hline \# Jumps & 289 & 169 & 171 & 200 & 139 & 297 & 95 \\
\hline \# Jump days & 231 & 134 & 128 & 161 & 111 & 246 & 72 \\
\hline$P($ Jump day $)$ & $16.08 \%$ & $9.32 \%$ & $8.91 \%$ & $11.20 \%$ & $7.72 \%$ & $17.12 \%$ & $5.01 \%$ \\
\hline P(Jump) & $0.47 \%$ & $0.27 \%$ & $0.27 \%$ & $0.32 \%$ & $0.22 \%$ & $0.48 \%$ & $0.15 \%$ \\
\hline Avg. jump size & $-23.84 \%$ & $-25.35 \%$ & $-14.85 \%$ & $-16.12 \%$ & $-28.15 \%$ & $-63.35 \%$ & $-0.07 \%$ \\
\hline Avg. positiv & $61.79 \%$ & $43.80 \%$ & $33.76 \%$ & $37.03 \%$ & $46.85 \%$ & $71.59 \%$ & $0.85 \%$ \\
\hline Avg. nega & $-86.40 \%$ & $-55.24 \%$ & $-27.81 \%$ & $-29.41 \%$ & $-77.26 \%$ & $-115.68 \%$ & $-0.83 \%$ \\
\hline$\%$ Negative jumps & $57.79 \%$ & $69.82 \%$ & $78.95 \%$ & $80.00 \%$ & $60.43 \%$ & $72.05 \%$ & $54.74 \%$ \\
\hline \multicolumn{8}{|l|}{ Medium-term options } \\
\hline \# Observations & 60,814 & 62,234 & 61,958 & 62,062 & 62,124 & 60,540 & 64,755 \\
\hline \# Days & 1,439 & 1,439 & 1,439 & 1,439 & 1,439 & 1,439 & 1439 \\
\hline \# Jumps & 228 & 129 & 159 & 106 & 150 & 263 & 92 \\
\hline \# Jump days & 181 & 100 & 111 & 80 & 125 & 218 & 70 \\
\hline$P($ Jump day $)$ & $12.58 \%$ & $6.95 \%$ & $7.71 \%$ & $5.56 \%$ & $8.69 \%$ & $15.15 \%$ & $4.86 \%$ \\
\hline$P($ Jump $)$ & $0.37 \%$ & $0.21 \%$ & $0.26 \%$ & $0.17 \%$ & $0.24 \%$ & $0.43 \%$ & $0.14 \%$ \\
\hline Avg. jump size & $-5.93 \%$ & $-7.80 \%$ & $-5.06 \%$ & $-12.97 \%$ & $-7.35 \%$ & $-29.60 \%$ & $-0.07 \%$ \\
\hline Avg. positive jump size & $34.40 \%$ & $29.28 \%$ & $26.09 \%$ & $24.49 \%$ & $34.78 \%$ & $49.32 \%$ & $0.84 \%$ \\
\hline Avg. negati & $-54.88 \%$ & $-34.50 \%$ & $-14.50 \%$ & $-19.63 \%$ & $-44.22 \%$ & $-80.78 \%$ & $-0.84 \%$ \\
\hline$\%$ Negative jumps & $45.18 \%$ & $58.14 \%$ & $76.73 \%$ & $84.91 \%$ & $53.33 \%$ & $60.84 \%$ & $55.43 \%$ \\
\hline \multicolumn{8}{|l|}{ Long-term options } \\
\hline \# Observations & 52,770 & 54,088 & 53,716 & 53,902 & 54,034 & 52,627 & 56,970 \\
\hline \# Days & 1,265 & 1,265 & 1,265 & 1,265 & 1,265 & 1,265 & \\
\hline \# Jumps & 223 & 106 & 145 & 72 & 118 & 296 & 86 \\
\hline \# Jump days & 159 & 80 & 112 & 62 & 97 & 222 & 67 \\
\hline$P($ Jump day $)$ & $12.57 \%$ & $6.32 \%$ & $8.85 \%$ & $4.90 \%$ & $7.66 \%$ & $17.54 \%$ & $5.29 \%$ \\
\hline$P(J u m p)$ & $0.42 \%$ & $0.20 \%$ & $0.27 \%$ & $0.13 \%$ & $0.22 \%$ & $0.56 \%$ & $0.15 \%$ \\
\hline Average jump size & $-8.95 \%$ & $-9.78 \%$ & $-5.98 \%$ & $-10.24 \%$ & $-27.73 \%$ & $-21.76 \%$ & $-0.08 \%$ \\
\hline Avg. positive jump size & $28.38 \%$ & $19.72 \%$ & $20.47 \%$ & $16.08 \%$ & $30.14 \%$ & $41.96 \%$ & $0.87 \%$ \\
\hline Avg. negative jump size & $-37.69 \%$ & $-33.28 \%$ & $-10.46 \%$ & $-15.50 \%$ & $-70.28 \%$ & $-62.82 \%$ & $-0.82 \%$ \\
\hline$\%$ Negative jumps & $56.50 \%$ & $55.66 \%$ & $85.52 \%$ & $83.33 \%$ & $57.63 \%$ & $60.81 \%$ & $55.81 \%$ \\
\hline
\end{tabular}

Entries report summary statistics for the detected jumps in 10-minute returns on E-mini options for any given delta and maturity bucket. The number of 10-minute return observations, days, detected jumps, jump days (days with at least one jump), and the probability of a jump day to occur P(Jump Day), the probability of a jump to occur $P(J u m p)$, the average size for positive jumps, the average size of negative jumps, and the number of negative jumps as a fraction of all jumps are reported. We use the Lee and Mykland (2008) jump detection methodology based on a significance level of $\alpha=0.1 \%$ to detect jumps. The sample period is January 1, 2005 to December 31, 2010. 
Table 4: Summary statistics of quoted sizes

\begin{tabular}{|c|c|c|c|c|c|c|}
\hline & $\begin{array}{c}\text { DOTM } \\
\text { puts }\end{array}$ & $\begin{array}{c}\text { OTM } \\
\text { puts }\end{array}$ & $\begin{array}{c}\text { ATM } \\
\text { puts }\end{array}$ & $\begin{array}{c}\text { ATM } \\
\text { calls }\end{array}$ & $\begin{array}{c}\text { OTM } \\
\text { calls }\end{array}$ & $\begin{array}{c}\text { DOTM } \\
\text { calls }\end{array}$ \\
\hline \multicolumn{7}{|c|}{ Panel A: Short-term options } \\
\hline Avg. bid size & 240.10 & 451.58 & 393.33 & 388.44 & 504.65 & 255.87 \\
\hline Avg. ask size & 226.79 & 437.54 & 364.30 & 355.92 & 462.23 & 233.12 \\
\hline Avg. trading volume & 98.22 & 105.1296 & 34.26 & 53.75 & 88.75 & 57.40 \\
\hline \multicolumn{7}{|l|}{ Conditional on jump } \\
\hline Avg. bid size & 90.69 & 172.15 & 220.94 & 233.18 & 171.01 & 137.14 \\
\hline Avg. ask size & 140.59 & 161.22 & 199.85 & 189.87 & 161.79 & 162.55 \\
\hline Avg. trading volume & 82.93 & 123.59 & 61.37 & 38.35 & 79.53 & 58.77 \\
\hline \multicolumn{7}{|c|}{ Panel B: Medium-term options } \\
\hline Avg. bid size & 293.08 & 484.19 & 377.78 & 375.43 & 477.77 & 266.47 \\
\hline Avg. ask size & 268.22 & 420.91 & 335.25 & 354.28 & 415.48 & 217.79 \\
\hline Avg. trading volume & 30.41 & 35.83 & 9.35 & 10.85 & 27.53 & 17.68 \\
\hline \multicolumn{7}{|c|}{ Conditional on jump } \\
\hline Avg. bid size & 159.13 & 223.07 & 245.57 & 273.67 & 178.37 & 144.79 \\
\hline Avg. ask size & 175.27 & 201.45 & 214.27 & 204.17 & 158.28 & 159.60 \\
\hline Avg. trading volume & 33.78 & 73.40 & 23.83 & 23.66 & 24.20 & 25.41 \\
\hline \multicolumn{7}{|c|}{ Panel C: Long-term options } \\
\hline Avg. bid size & 351.33 & 417.40 & 347.47 & 336.48 & 420.45 & 270.39 \\
\hline Avg. ask size & 306.99 & 359.16 & 305.73 & 312.79 & 347.60 & 234.67 \\
\hline Avg. trading volume & 11.29 & 15.04 & 4.39 & 5.00 & 10.17 & 6.86 \\
\hline \multicolumn{7}{|c|}{ Conditional on jump } \\
\hline Avg. bid size & 158.91 & 245.07 & 266.19 & 241.86 & 180.94 & 154.00 \\
\hline Avg. ask size & 174.88 & 198.63 & 202.72 & 222.64 & 153.53 & 146.14 \\
\hline Avg. trading volume & 14.62 & 59.42 & 17.46 & 3.47 & 22.17 & 4.96 \\
\hline
\end{tabular}

Entries report the average bid and ask trade sizes at the best bid and ask prices, respectively, for any given delta and maturity category. Figures are reported separately for all observations and the jumprelated observations. Jumps have been detected using the Lee and Mykland (2008) jump detection methodology based on a significance level of $\alpha=0.1 \%$. The average trade size is also reported. The sample spans January 1, 2005 to December 31, 2010. 
Table 5: Relation between jumps and scheduled news announcements

\begin{tabular}{ccccccc}
\hline $\begin{array}{c}\text { DOTM } \\
\text { puts }\end{array}$ & $\begin{array}{c}\text { OTM } \\
\text { puts }\end{array}$ & $\begin{array}{c}\text { ATM } \\
\text { puts }\end{array}$ & $\begin{array}{c}\text { ATM } \\
\text { calls }\end{array}$ & $\begin{array}{c}\text { OTM } \\
\text { calls }\end{array}$ & $\begin{array}{c}\text { DOTM } \\
\text { calls }\end{array}$ & Futures \\
\hline
\end{tabular}

Panel A: Aggregated over all news items

Short-term options

\# Jumps within

10 mins of news

$P($ News $\mid$ Jump $)$

60

42

40

57

39

61

23

P(Jump $\mid$ News $)$

$6.75 \% \quad 4.72 \% \quad 4.50 \%$

$28.50 \% \quad 28.06 \%$

$20.54 \%$

$24.21 \%$

Medium-term options

\# Jumps within

10 mins of news

$P(N e w s \mid J u m p)$

34
$14.91 \%$

35

25

$6.41 \%$

$4.39 \%$

$6.86 \%$

$2.59 \%$

$P($ Jump $\mid$ News $)$

$3.82 \%$

$27.13 \%$

$15.72 \%$

19

35

43

25

$3.94 \% \quad 2.81 \%$

$17.92 \% \quad 23.33 \%$

$16.35 \%$

$27.17 \%$

$P(J u m p \mid N e w s)$

Long-term options

\# Jumps within

10 mins of news

$2.14 \%$

$3.94 \%$

$4.84 \%$

$2.81 \%$

$\begin{array}{llllllll}P \text { (News|Jump }) & 14.35 \% & 19.81 \% & 14.48 \% & 16.67 \% & 27.97 \% & 14.86 \% & 26.74 \%\end{array}$

$P($ Jump $\mid$ News $)$

$14.35 \%$

$2.36 \%$

$2.36 \%$

$1.35 \%$

$3.71 \%$

$4.95 \%$

$2.59 \%$

Continued on next page 
Table 5: Relation between jumps and scheduled news announcements Continued from previous page

\begin{tabular}{|c|c|c|c|c|c|c|c|}
\hline & $\begin{array}{c}\text { DOTM } \\
\text { puts }\end{array}$ & $\begin{array}{l}\text { OTM } \\
\text { puts }\end{array}$ & $\begin{array}{c}\text { ATM } \\
\text { puts }\end{array}$ & $\begin{array}{c}\text { ATM } \\
\text { calls }\end{array}$ & $\begin{array}{c}\text { OTM } \\
\text { calls }\end{array}$ & $\begin{array}{c}\text { DOTM } \\
\text { calls }\end{array}$ & Futures \\
\hline \multicolumn{8}{|c|}{ Panel B: P(News|Jump) disaggregated by news items } \\
\hline \multicolumn{8}{|c|}{ Short-term options } \\
\hline NFP & $5.19 \%$ & $8.88 \%$ & $6.43 \%$ & $6.00 \%$ & $5.04 \%$ & $4.38 \%$ & $11.58 \%$ \\
\hline CCI & $0.35 \%$ & $0.00 \%$ & $1.75 \%$ & $1.50 \%$ & $0.00 \%$ & $1.35 \%$ & $0.00 \%$ \\
\hline CPI & $2.42 \%$ & $2.37 \%$ & $2.92 \%$ & $3.50 \%$ & $4.32 \%$ & $0.67 \%$ & $0.00 \%$ \\
\hline DGO & $2.08 \%$ & $0.59 \%$ & $2.92 \%$ & $2.00 \%$ & $1.44 \%$ & $2.02 \%$ & $0.00 \%$ \\
\hline FOMC & $0.69 \%$ & $1.18 \%$ & $2.34 \%$ & $1.50 \%$ & $2.16 \%$ & $0.67 \%$ & $9.47 \%$ \\
\hline GDP & $2.08 \%$ & $1.78 \%$ & $1.75 \%$ & $3.50 \%$ & $2.88 \%$ & $2.69 \%$ & $1.05 \%$ \\
\hline IJC & $7.96 \%$ & $5.92 \%$ & $7.60 \%$ & $9.50 \%$ & $11.51 \%$ & $8.75 \%$ & $1.05 \%$ \\
\hline LI & $0.35 \%$ & $0.00 \%$ & $0.58 \%$ & $0.50 \%$ & $0.00 \%$ & $0.34 \%$ & $0.00 \%$ \\
\hline NHS & $0.35 \%$ & $0.59 \%$ & $0.00 \%$ & $1.00 \%$ & $0.00 \%$ & $0.67 \%$ & $1.05 \%$ \\
\hline PPI & $1.73 \%$ & $4.14 \%$ & $0.00 \%$ & $1.50 \%$ & $1.44 \%$ & $1.68 \%$ & $0.00 \%$ \\
\hline RSA & $1.04 \%$ & $3.55 \%$ & $0.00 \%$ & $1.00 \%$ & $2.88 \%$ & $1.01 \%$ & $0.00 \%$ \\
\hline \multicolumn{8}{|c|}{ Medium-term options } \\
\hline NFP & $6.14 \%$ & $8.53 \%$ & $5.66 \%$ & $5.66 \%$ & $10.00 \%$ & $2.28 \%$ & $14.13 \%$ \\
\hline CCI & $0.00 \%$ & $0.78 \%$ & $1.89 \%$ & $1.89 \%$ & $0.67 \%$ & $0.38 \%$ & $0.00 \%$ \\
\hline CPI & $0.88 \%$ & $0.78 \%$ & $1.89 \%$ & $0.94 \%$ & $1.33 \%$ & $2.28 \%$ & $0.00 \%$ \\
\hline DGO & $0.44 \%$ & $1.55 \%$ & $0.63 \%$ & $0.00 \%$ & $2.67 \%$ & $1.52 \%$ & $0.00 \%$ \\
\hline FOMC & $1.75 \%$ & $3.10 \%$ & $2.52 \%$ & $1.89 \%$ & $1.33 \%$ & $0.76 \%$ & $9.78 \%$ \\
\hline GDP & $0.44 \%$ & $3.88 \%$ & $0.00 \%$ & $1.89 \%$ & $1.33 \%$ & $0.76 \%$ & $1.09 \%$ \\
\hline IJC & $3.07 \%$ & $10.08 \%$ & $1.89 \%$ & $4.72 \%$ & $5.33 \%$ & $6.08 \%$ & $1.09 \%$ \\
\hline LI & $0.44 \%$ & $0.78 \%$ & $0.00 \%$ & $0.00 \%$ & $0.67 \%$ & $1.14 \%$ & $0.00 \%$ \\
\hline NHS & $0.00 \%$ & $0.00 \%$ & $0.63 \%$ & $1.89 \%$ & $0.67 \%$ & $0.76 \%$ & $1.09 \%$ \\
\hline PPI & $1.32 \%$ & $0.78 \%$ & $0.63 \%$ & $0.00 \%$ & $0.00 \%$ & $0.76 \%$ & $0.00 \%$ \\
\hline RSA & $0.44 \%$ & $1.55 \%$ & $0.00 \%$ & $0.94 \%$ & $0.67 \%$ & $0.76 \%$ & $0.00 \%$ \\
\hline \multicolumn{8}{|c|}{ Long-term options } \\
\hline NFP & $3.14 \%$ & $6.60 \%$ & $4.14 \%$ & $5.56 \%$ & $7.63 \%$ & $3.04 \%$ & $10.47 \%$ \\
\hline CCI & $1.35 \%$ & $1.89 \%$ & $2.07 \%$ & $0.00 \%$ & $3.39 \%$ & $0.00 \%$ & $2.33 \%$ \\
\hline CPI & $0.90 \%$ & $0.00 \%$ & $1.38 \%$ & $0.00 \%$ & $5.93 \%$ & $1.01 \%$ & $0.00 \%$ \\
\hline DGO & $2.69 \%$ & $0.00 \%$ & $0.69 \%$ & $0.00 \%$ & $0.85 \%$ & $1.69 \%$ & $0.00 \%$ \\
\hline FOMC & $0.45 \%$ & $3.77 \%$ & $3.45 \%$ & $1.39 \%$ & $1.69 \%$ & $1.35 \%$ & $10.47 \%$ \\
\hline GDP & $0.90 \%$ & $0.94 \%$ & $0.00 \%$ & $1.39 \%$ & $3.39 \%$ & $1.69 \%$ & $1.16 \%$ \\
\hline IJC & $4.04 \%$ & $5.66 \%$ & $0.69 \%$ & $2.78 \%$ & $8.47 \%$ & $3.72 \%$ & $1.16 \%$ \\
\hline LI & $1.35 \%$ & $0.00 \%$ & $0.00 \%$ & $0.00 \%$ & $0.00 \%$ & $0.68 \%$ & $0.00 \%$ \\
\hline NHS & $0.90 \%$ & $0.94 \%$ & $2.07 \%$ & $1.39 \%$ & $0.00 \%$ & $0.34 \%$ & $1.16 \%$ \\
\hline PPI & $0.45 \%$ & $0.94 \%$ & $0.00 \%$ & $0.00 \%$ & $0.85 \%$ & $1.35 \%$ & $0.00 \%$ \\
\hline RSA & $0.45 \%$ & $0.94 \%$ & $0.00 \%$ & $5.56 \%$ & $0.00 \%$ & $2.36 \%$ & $0.00 \%$ \\
\hline
\end{tabular}


Table 5: Relation between jumps and scheduled news announcements Continued from previous page

\begin{tabular}{ccccccc}
\hline $\begin{array}{c}\text { DOTM } \\
\text { puts }\end{array}$ & $\begin{array}{c}\text { OTM } \\
\text { puts }\end{array}$ & $\begin{array}{c}\text { ATM } \\
\text { puts }\end{array}$ & $\begin{array}{c}\text { ATM } \\
\text { calls }\end{array}$ & $\begin{array}{c}\text { OTM } \\
\text { calls }\end{array}$ & $\begin{array}{c}\text { DOTM } \\
\text { calls }\end{array}$ & Futures \\
\hline
\end{tabular}

Panel C: $P($ Jump $\mid$ News $)$ disaggregated by news items

Short-term options

$\begin{array}{lccccccc}\text { NFP } & 20.83 \% & 20.83 \% & 15.28 \% & 16.67 \% & 9.72 \% & 18.06 \% & 15.28 \% \\ \text { CCI } & 1.39 \% & 0.00 \% & 4.17 \% & 4.17 \% & 0.00 \% & 5.56 \% & 0.00 \% \\ \text { CPI } & 9.72 \% & 5.56 \% & 6.94 \% & 9.72 \% & 8.33 \% & 2.78 \% & 0.00 \% \\ \text { DGO } & 8.33 \% & 1.39 \% & 6.94 \% & 5.56 \% & 2.78 \% & 8.33 \% & 0.00 \% \\ \text { FOMC } & 4.00 \% & 4.00 \% & 8.00 \% & 6.00 \% & 6.00 \% & 4.00 \% & 18.00 \% \\ \text { GDP } & 8.33 \% & 4.17 \% & 4.17 \% & 9.72 \% & 5.56 \% & 11.11 \% & 1.39 \% \\ \text { IJC } & 7.35 \% & 3.19 \% & 4.15 \% & 6.07 \% & 5.11 \% & 8.31 \% & 0.32 \% \\ \text { LI } & 1.39 \% & 0.00 \% & 1.39 \% & 1.39 \% & 0.00 \% & 1.39 \% & 0.00 \% \\ \text { NHS } & 1.39 \% & 1.39 \% & 0.00 \% & 2.78 \% & 0.00 \% & 2.78 \% & 1.39 \% \\ \text { PPI } & 6.94 \% & 9.72 \% & 0.00 \% & 4.17 \% & 2.78 \% & 6.94 \% & 0.00 \% \\ \text { RSA } & 4.17 \% & 8.33 \% & 0.00 \% & 2.78 \% & 5.56 \% & 4.17 \% & 0.00 \%\end{array}$

Medium-term options

$\begin{array}{lccccccc}\text { NFP } & 19.44 \% & 15.28 \% & 12.50 \% & 8.33 \% & 20.83 \% & 8.33 \% & 18.06 \% \\ \text { CCI } & 0.00 \% & 1.39 \% & 4.17 \% & 2.78 \% & 1.39 \% & 1.39 \% & 0.00 \% \\ \text { CPI } & 2.78 \% & 1.39 \% & 4.17 \% & 1.39 \% & 2.78 \% & 8.33 \% & 0.00 \% \\ \text { DGO } & 1.39 \% & 2.78 \% & 1.39 \% & 0.00 \% & 5.56 \% & 5.56 \% & 0.00 \% \\ \text { FOMC } & 8.00 \% & 8.00 \% & 8.00 \% & 4.00 \% & 4.00 \% & 4.00 \% & 18.00 \% \\ \text { GDP } & 1.39 \% & 6.94 \% & 0.00 \% & 2.78 \% & 2.78 \% & 2.78 \% & 1.39 \% \\ \text { IJC } & 2.24 \% & 4.15 \% & 0.96 \% & 1.60 \% & 2.56 \% & 5.11 \% & 0.32 \% \\ \text { LI } & 1.39 \% & 1.39 \% & 0.00 \% & 0.00 \% & 1.39 \% & 4.17 \% & 0.00 \% \\ \text { NHS } & 0.00 \% & 0.00 \% & 1.39 \% & 2.78 \% & 1.39 \% & 2.78 \% & 1.39 \% \\ \text { PPI } & 4.17 \% & 1.39 \% & 1.39 \% & 0.00 \% & 0.00 \% & 2.78 \% & 0.00 \% \\ \text { RSA } & 1.39 \% & 2.78 \% & 0.00 \% & 1.39 \% & 1.39 \% & 2.78 \% & 0.00 \%\end{array}$

\section{Long-term options}

\begin{tabular}{lccccccc} 
NFP & $9.72 \%$ & $9.72 \%$ & $8.33 \%$ & $5.56 \%$ & $12.50 \%$ & $12.50 \%$ & $12.50 \%$ \\
CCI & $4.17 \%$ & $2.78 \%$ & $4.17 \%$ & $0.00 \%$ & $5.56 \%$ & $0.00 \%$ & $2.78 \%$ \\
CPI & $2.78 \%$ & $0.00 \%$ & $2.78 \%$ & $0.00 \%$ & $9.72 \%$ & $4.17 \%$ & $0.00 \%$ \\
DGO & $8.33 \%$ & $0.00 \%$ & $1.39 \%$ & $0.00 \%$ & $1.39 \%$ & $6.94 \%$ & $0.00 \%$ \\
FOMC & $2.00 \%$ & $8.00 \%$ & $10.00 \%$ & $2.00 \%$ & $4.00 \%$ & $8.00 \%$ & $18.00 \%$ \\
GDP & $2.78 \%$ & $1.39 \%$ & $0.00 \%$ & $1.39 \%$ & $5.56 \%$ & $6.94 \%$ & $1.39 \%$ \\
IJC & $2.88 \%$ & $1.92 \%$ & $0.32 \%$ & $0.64 \%$ & $3.19 \%$ & $3.51 \%$ & $0.32 \%$ \\
LI & $4.17 \%$ & $0.00 \%$ & $0.00 \%$ & $0.00 \%$ & $0.00 \%$ & $2.78 \%$ & $0.00 \%$ \\
NHS & $2.78 \%$ & $1.39 \%$ & $4.17 \%$ & $1.39 \%$ & $0.00 \%$ & $1.39 \%$ & $1.39 \%$ \\
PPI & $1.39 \%$ & $1.39 \%$ & $0.00 \%$ & $0.00 \%$ & $1.39 \%$ & $5.56 \%$ & $0.00 \%$ \\
RSA & $1.39 \%$ & $1.39 \%$ & $0.00 \%$ & $5.56 \%$ & $0.00 \%$ & $9.72 \%$ & $0.00 \%$ \\
\hline
\end{tabular}

Entries report summary statistics on the relation between detected jumps and scheduled macroeconomic news announcement items for all investigated delta and maturity categories. The probability of a jump being related to a specific news announcement $P(N e w s \mid J u m p)$ and the probability of a news announcement leading to a jump $P($ Jump $\mid$ News $)$ are reported. Panel A reports these statistics aggregated over all considered news announcement items and Panels $\mathrm{B}$ and $\mathrm{C}$ report them disaggregated by individual announcement items. Jumps have been detected using the Lee and Mykland (2008) jump detection methodology based on a significance level of $\alpha=0.1 \%$. A jump is defined to be related to news if it occurred within \pm 10 minutes of a scheduled news announcement. 
Table 6: Information shocks and illiquidity as jump determinants

\begin{tabular}{lccc}
\hline & Short-term & Medium-term & Long-term \\
\hline \multicolumn{4}{c}{ Panel A : News covariates } \\
$c$ & $-3.8579^{* * *}$ & $-4.1575^{* * *}$ & $-4.8081^{* * *}$ \\
$N F P_{t}$ & $0.7506^{* * *}$ & $0.6315^{* *}$ & -0.0617 \\
$C C I_{t}$ & -1.0005 & -0.6084 & -0.0903 \\
$C P I_{t}$ & 0.2371 & -0.2751 & 0.2545 \\
$D G O_{t}$ & 0.1317 & -0.9814 & -1.1075 \\
$F O M C_{t}$ & - & - & - \\
$G D P_{t}$ & 0.1568 & -0.1131 & $0.7241^{* * *}$ \\
$I J C_{t}$ & $0.287^{*}$ & -0.0701 & 0.1289 \\
$L I_{t}$ & -0.9801 & -0.6891 & - \\
$N H S_{t}$ & - & -6.6603 & - \\
$P P I_{t}$ & -0.0452 & -0.137 & -0.0892 \\
$R S A_{S} t$ & -0.1407 & -1.0811 & $0.7789^{* *}$
\end{tabular}

Panel B: News and liquidity covariates

\begin{tabular}{lccc}
$c$ & $-3.4309^{* * *}$ & $-3.7201^{* * *}$ & $-4.1162^{* * *}$ \\
sBA $_{t-1}$ & $0.1161^{* * *}$ & $0.1431^{* * *}$ & $0.1737^{* * *}$ \\
BidSize $_{t-1}$ & $-0.0026^{* *}$ & -0.002 & -0.0025 \\
AskSize $_{t-1}$ & -0.0015 & -0.0018 & -0.0035 \\
NFP & $0.5904^{* * *}$ & $0.5042^{* *}$ & -0.2296 \\
CCI & -0.4622 & -0.2161 & 0.2941 \\
CPI & 0.1338 & -0.3598 & 0.0227 \\
$D G O_{t}$ & 0.0592 & -1.0874 & -1.3451 \\
$F O M C_{t}$ & - & - & - \\
$G D P_{t}$ & -0.0079 & -0.3073 & $0.6001^{* *}$ \\
$I J C_{t}$ & 0.1776 & -0.2565 & -0.1036 \\
LI $_{t}$ & -0.4828 & -0.3253 & - \\
$N H S_{t}$ & - & -4.9552 & - \\
$P P I_{t}$ & -0.1628 & -0.2543 & -0.3161 \\
RSA $_{t}$ & -0.2575 & -1.0971 & 0.4082 \\
\hline
\end{tabular}

Entries report the estimation results for the logistic regression models in equations (8) and (9) (Panels $\mathrm{A}$ and $\mathrm{B}$, respectively). The estimation is performed separately for short-, medium-, and long-term options on a sample pooled across all delta categories. Only news-related observations are considered. The estimation is performed via maximum likelihood and ${ }^{* *}, * *$, and $*$ report statistical significance at the 1\%,5\%, and 10\% levels, respectively. The sample period is January 1, 2005 to December 31, 2010 . 
Table 7: Information shocks, volume, and illiquidity as jump determinants

\begin{tabular}{|c|c|c|c|}
\hline & Short-term & Medium-term & Long-term \\
\hline \multicolumn{4}{|c|}{ Panel A: News, volume, and liquidity covariates } \\
\hline$c$ & $-3.4042^{* * *}$ & $-3.6822^{* * *}$ & $-4.0221^{* * *}$ \\
\hline$s B A_{t-1}$ & $0.1142^{* * *}$ & $0.1463^{* * *}$ & $0.1703^{* * *}$ \\
\hline BidSize $_{t-1}$ & $-0.0025^{* *}$ & -0.0021 & -0.0026 \\
\hline AskSize $_{t-1}$ & -0.0014 & -0.0019 & -0.0037 \\
\hline Volume $_{t-1, t}$ & -0.002 & 0.0024 & 0.0011 \\
\hline$N F P_{t}$ & $0.5879^{* * *}$ & $0.4857^{*}$ & -0.2329 \\
\hline$C C I_{t}$ & -0.3124 & -0.3031 & 0.2957 \\
\hline$C P I_{t}$ & 0.1126 & -0.3878 & 0.0331 \\
\hline$D G O_{t}$ & 0.0448 & -1.1291 & -1.4186 \\
\hline$F O M C_{t}$ & - & - & - \\
\hline$G D P_{t}$ & -0.0156 & -0.3327 & $0.5768^{* *}$ \\
\hline$I J C_{t}$ & 0.1623 & -0.2235 & -0.1085 \\
\hline$L I_{t}$ & -0.3449 & -0.3942 & - \\
\hline$N H S_{t}$ & - & -5.4217 & - \\
\hline$P P I_{t}$ & -0.1783 & -0.28 & -0.304 \\
\hline$R S A_{t}$ & -0.2734 & -1.1052 & 0.4241 \\
\hline
\end{tabular}

Panel B: Volume and liquidity covariates for no-news related jumps

$\begin{array}{lccc}c & -5.2846^{* * *} & -5.3888^{* * *} & -5.1133^{* * *} \\ \text { sBA }_{t-1} & 0.3338^{* * *} & 0.292^{* * *} & 0.2353^{* * *} \\ \text { BidSize }_{t-1} & -0.0028^{* * *} & -0.0019^{* * *} & -0.0023^{* * *} \\ \text { AskSize }_{t-1} & -0.0022^{* * *} & -0.0024^{* * *} & -0.0025^{* * *} \\ \text { V }_{\text {olume }} t-1, t & 0.0006^{* * *} & 0.0007^{* * *} & 0.0013^{* * *}\end{array}$

Entries report the estimation results for the logistic regression models in equations (11) and (12) (Panels A and B, respectively). The estimation is performed separately for short-, medium-, and long-term options on a sample pooled across all delta categories. Panel A considers only news-related observations and Panel B considers only non-news-related observations. The estimation is performed via maximum likelihood and ${ }^{* * *}, * *$, and $*$ report statistical significance at the $1 \%, 5 \%$, and $10 \%$ levels, respectively. The sample period is January 1, 2005 to December 31, 2010. 
Table 8: Summary statistics of detected jumps (non-crisis and crisis subsamples)

\begin{tabular}{ccccccc}
\hline DOTM & OTM & ATM & ATM & OTM & DOTM & Futures \\
puts & puts & puts & calls & $\begin{array}{c}\text { calls } \\
\text { calls }\end{array}$ & \\
\hline
\end{tabular}

Panel A: Non-crisis subsample

Short-term options

$\begin{array}{lccccccc}\text { \# Observations } & 25,501 & 26,419 & 26,044 & 26,122 & 26,359 & 24,912 & 27,720 \\ \text { \# Jumps } & 149 & 98 & 54 & 101 & 88 & 115 & 40 \\ \text { \# Jump days } & 124 & 78 & 46 & 85 & 68 & 95 & 32 \\ \text { P(Jump day } & 20.13 \% & 12.66 \% & 7.47 \% & 13.80 \% & 11.04 \% & 15.42 \% & 5.19 \% \\ \text { P(Jump) } & 0.58 \% & 0.37 \% & 0.21 \% & 0.39 \% & 0.33 \% & 0.46 \% & 0.14 \% \\ \text { Avg. jump size } & -31.44 \% & -30.79 \% & -19.93 \% & -21.31 \% & -36.98 \% & -35.99 \% & 0.00 \% \\ \text { \% Negative jumps } & 59.73 \% & 71.43 \% & 85.19 \% & 83.17 \% & 62.50 \% & 66.96 \% & 50.00 \% \\ & & & & & & & \\ \text { Medium-term options } & & & & & & & \\ \text { \# Observations } & 24,237 & 25,356 & 25,092 & 25,204 & 25,262 & 23,937 & 27,540 \\ \text { \# Jumps } & 120 & 55 & 47 & 54 & 89 & 98 & 39 \\ \text { \# Jump days } & 97 & 45 & 36 & 44 & 75 & 83 & 31 \\ \text { P(Jump day) } & 15.85 \% & 7.35 \% & 5.88 \% & 7.19 \% & 12.25 \% & 13.56 \% & 5.07 \% \\ \text { P(Jump) } & 0.50 \% & 0.22 \% & 0.19 \% & 0.21 \% & 0.35 \% & 0.41 \% & 0.14 \% \\ \text { Avg. jump size } & -5.49 \% & -11.71 \% & -14.79 \% & -10.78 \% & -4.17 \% & -16.26 \% & 0.00 \% \\ \text { \% Negative jumps } & 46.67 \% & 65.45 \% & 95.74 \% & 87.04 \% & 47.19 \% & 50.00 \% & 51.28 \%\end{array}$

Long-term options

\begin{tabular}{lccccccc} 
\# Observations & 17,569 & 18,427 & 18,082 & 18,252 & 18,369 & 17,431 & 20,700 \\
\# Jumps & 116 & 38 & 43 & 37 & 44 & 103 & 36 \\
\# Jump days & 77 & 28 & 36 & 35 & 36 & 79 & 30 \\
P(Jump day) & $16.74 \%$ & $6.09 \%$ & $7.83 \%$ & $7.63 \%$ & $7.83 \%$ & $17.17 \%$ & $6.52 \%$ \\
P(Jump) & $0.66 \%$ & $0.21 \%$ & $0.24 \%$ & $0.20 \%$ & $0.24 \%$ & $0.59 \%$ & $0.17 \%$ \\
Avg. jump size & $-3.60 \%$ & $-2.04 \%$ & $-9.34 \%$ & $-7.08 \%$ & $-5.81 \%$ & $-21.31 \%$ & $-0.02 \%$ \\
\% Negative jumps & $48.28 \%$ & $55.26 \%$ & $93.02 \%$ & $86.49 \%$ & $61.36 \%$ & $53.40 \%$ & $52.78 \%$ \\
\hline
\end{tabular}

Continued on next page 
Table 8: Summary statistics of detected jumps (non-crisis and crisis subsamples) Continued from previous page

\begin{tabular}{ccccccc}
\hline DOTM & OTM & ATM & ATM & OTM & DOTM & Futures \\
puts & puts & puts & calls & calls & calls & \\
\hline
\end{tabular}

\section{Panel B: Crisis subsample}

\section{Short-term options}

\# Observations
\# Jumps
\# Jump days
P(Jump day $)$
P(Jump)
Avg. jump size
$\%$ Negative jumps

36,596
140
107
$13.05 \%$
$0.38 \%$
$-32.31 \%$
$60.71 \%$

$\begin{array}{cc}36,678 & 36,645 \\ 71 & 117 \\ 56 & 82 \\ 6.83 \% & 10.00 \% \\ 0.19 \% & 0.32 \% \\ -30.03 \% & -17.59 \% \\ 71.83 \% & 82.91 \%\end{array}$

36,648
99
76
$9.27 \%$
$0.27 \%$
$-21.40 \%$
$82.83 \%$

36,670

36,592

51

43

$5.24 \%$

$0.14 \%$

$-56.32 \%$

$72.55 \%$

182

36,900

151

$18.41 \%$

$0.50 \%$

$-50.81 \%$

$70.88 \%$

55

40

$4.88 \%$

$0.15 \%$

$0.06 \%$

$47.27 \%$

\section{Medium-term options}

\# Observations $\quad 36,532$

\# Jumps

107

\# Jump days

83

P(Jump day)

$10.05 \%$

36,833

36,821

36,813

36,817

36,558

37,170

$74 \quad 112$

55

75

52

61

165

53

$6.66 \% \quad 9.08 \%$

36

50

135

39

$P$ (Jump)

$0.29 \%$

$0.20 \%$

$0.30 \%$

$4.36 \%$

$6.05 \%$

$16.34 \%$

$4.72 \%$

Avg. jump size

$-3.13 \%$

$-9.71 \%$

$-5.89 \%$

$0.14 \%$

$0.17 \%$

$0.45 \%$

$0.14 \%$

$\%$ Negative jumps

$45.79 \%$

$66.22 \%$

$80.36 \%$

$-12.40 \%$

$-12.36 \%$

$-29.17 \%$

$0.06 \%$

$90.38 \%$

$57.38 \%$

$60.61 \%$

$49.06 \%$

\section{Long-term options}

\# Observations

35,156

35,616

35,59

35,606

35,620

35,151

36,225

\# Jumps

107

\# Jump days

82

$P$ (Jump day)

$10.20 \%$

68

102

35

74

193

50

27

61

143

37

$P$ (Jump)

$0.30 \%$

$6.46 \%$

$9.44 \%$

$3.35 \%$

$7.58 \%$

$17.76 \%$

$4.60 \%$

Avg. jump size

$-3.87 \%$

$0.19 \%$

$0.29 \%$

$0.10 \%$

$0.21 \%$

$0.55 \%$

$0.14 \%$

$-7.75 \%$

$-5.59 \%$

$-7.77 \%$

$-17.44 \%$

$-28.65 \%$

$0.01 \%$

$61.76 \% \quad 87.25 \%$

$88.57 \%$

$54.05 \%$

$62.69 \%$

$50.00 \%$

Entries report summary statistics for the detected jumps for all investigated delta and maturity categories over the non-crisis subsample (Panel A) and crisis subsample (Panel B). The number of detected jumps, the number of jump days (days with at least one jump), the probability of a jump day to occur $P($ Jump Day), the probability of a jump to occur $P(J u m p)$ and the number of negative jumps as a fraction of all jumps are reported. Jumps have been detected using the Lee and Mykland (2008) jump detection methodology based on a significance level of $\alpha=0.1 \%$. The sample period is January 1, 2005 to July 31, 2007 for the non-crisis subsample and August 1, 2007 to December 31, 2010 for the crisis subsample. 
Table 9: Relation between jumps and scheduled news announcements (non-crisis and crisis subsamples)

\begin{tabular}{ccccccc}
\hline $\begin{array}{c}\text { DOTM } \\
\text { puts }\end{array}$ & $\begin{array}{c}\text { OTM } \\
\text { puts }\end{array}$ & $\begin{array}{c}\text { ATM } \\
\text { puts }\end{array}$ & $\begin{array}{c}\text { ATM } \\
\text { calls }\end{array}$ & $\begin{array}{c}\text { OTM } \\
\text { calls }\end{array}$ & $\begin{array}{c}\text { DOTM } \\
\text { calls }\end{array}$ & Futures \\
\hline
\end{tabular}

Panel A: Non-crisis subsample

Short-term options

\# Jumps within

10 mins of news

$P($ News $\mid$ Jump $)$

19

$20.41 \%$

9

26

18

14

11

$P($ Jump $\mid$ News $)$

$12.75 \%$

$5.21 \%$

$16.67 \%$

$25.74 \%$

$20.45 \%$

$12.17 \%$

$27.50 \%$

Medium-term options

\# Jumps within

10 mins of news

$P($ News $\mid$ Jump $)$

11

1

$\begin{array}{cc}11 & 6 \\ 20.00 \% & 12.77 \%\end{array}$

5

15

10

12

P(Jump $\mid$ News $)$

$2.86 \%$

$2.86 \%$

$1.56 \%$

$1.30 \%$

$16.85 \%$

$10.20 \%$

$30.77 \%$

Long-term options

\# Jumps within

10 mins of news

9
$7.76 \%$

5

7

1

9

9

12

$P($ News $\mid$ Jump $)$

$2.34 \%$

$13.16 \%$

$16.28 \%$

$2.70 \%$

$20.45 \%$

$8.74 \%$

$33.33 \%$

$P($ Jump $\mid$ News $)$

$1.30 \%$

$1.82 \%$

$0.26 \%$

$2.34 \%$

$2.34 \%$

$3.13 \%$

Panel B: Crisis subsample

Short-term options

\# Jumps within

10 mins of news

$P($ News $\mid$ Jump $)$

41

22

31

31

21

47

12

$P($ Jump $\mid$ News $)$

$29.29 \%$

$30.99 \%$

$26.50 \%$

$31.31 \%$

$41.18 \%$

$25.82 \%$

$21.82 \%$

$8.13 \%$

$4.37 \%$

$6.15 \%$

$6.15 \%$

$4.17 \%$

$9.33 \%$

$2.38 \%$

Medium-term options

\# Jumps within

10 mins of news

$P$ (News $\mid$ Jump $) \quad 21.50 \%$

24

19

14

20

33

$20.00 \%$

13

P(Jump $\mid$ News $)$

$4.76 \%$

$3.77 \%$

$2.78 \%$

$32.79 \%$

$6.55 \%$

$24.53 \%$

$2.58 \%$

Long-term options

\# Jumps within

10 mins of news

23

16

14

11

24

35

11

$P($ News $\mid$ Jump $)$

$21.50 \% \quad 23.53 \%$

$13.73 \%$

$31.43 \%$

$32.43 \%$

$18.13 \%$

$22.00 \%$

P(Jump $\mid$ News $)$

$4.56 \% \quad 3.17 \%$

$2.78 \%$

$2.18 \% \quad 4.76 \%$

$6.94 \%$

$2.18 \%$

Entries report summary statistics on the relation between detected jumps and macroeconomic news announcements for all investigated delta and maturity categories over the non-crisis subsample (Panel A) and crisis subsample (Panel B). The number of jumps that occurred within \pm 10 minutes of a scheduled news announcement, the probability of a news announcement leading to a jump $P($ Jump $\mid$ News $)$ as well as the probability of a jump being related to a news announcement $P(N e w s \mid J u m p)$ are reported. Jumps have been detected using the Lee and Mykland (2008) jump detection methodology based on a significance level of $\alpha=0.1 \%$. The sample period is January 1, 2005 to July 31, 2007 for the non-crisis and August 1, 2007 to December 31, 2010 for the crisis subsample. 
Table 10: Relation between jumps and scheduled news announcements disaggregated by announcement items (non-crisis and crisis subsamples)

\begin{tabular}{ccccccc}
\hline $\begin{array}{c}\text { DOTM } \\
\text { puts }\end{array}$ & $\begin{array}{c}\text { OTM } \\
\text { puts }\end{array}$ & $\begin{array}{c}\text { ATM } \\
\text { puts }\end{array}$ & $\begin{array}{c}\text { ATM } \\
\text { calls }\end{array}$ & $\begin{array}{c}\text { OTM } \\
\text { calls }\end{array}$ & $\begin{array}{c}\text { DOTM } \\
\text { calls }\end{array}$ & Futures \\
\hline
\end{tabular}

Panel A: $P(N e w s \mid J u m p)$ over the non-crisis subsample

Short-term options

$\begin{array}{lccccccc}\text { NFP } & 1.34 \% & 4.08 \% & 5.56 \% & 1.98 \% & 2.27 \% & 1.74 \% & 10.00 \% \\ \text { CCI } & 0.67 \% & 0.00 \% & 1.85 \% & 0.00 \% & 0.00 \% & 2.61 \% & 0.00 \% \\ \text { CPI } & 2.68 \% & 3.06 \% & 3.70 \% & 4.95 \% & 2.27 \% & 0.87 \% & 0.00 \% \\ \text { DGO } & 2.01 \% & 0.00 \% & 1.85 \% & 0.00 \% & 2.27 \% & 0.87 \% & 0.00 \% \\ \text { FOMC } & 0.00 \% & 0.00 \% & 1.85 \% & 0.99 \% & 0.00 \% & 0.00 \% & 12.50 \% \\ \text { GDP } & 2.68 \% & 0.00 \% & 1.85 \% & 2.97 \% & 1.14 \% & 0.00 \% & 0.00 \% \\ \text { IJC } & 3.36 \% & 8.16 \% & 0.00 \% & 11.88 \% & 10.23 \% & 3.48 \% & 2.50 \% \\ \text { LI } & 0.00 \% & 0.00 \% & 0.00 \% & 0.00 \% & 0.00 \% & 0.00 \% & 0.00 \% \\ \text { NHS } & 0.00 \% & 0.00 \% & 0.00 \% & 0.99 \% & 0.00 \% & 0.00 \% & 2.50 \% \\ \text { PPI } & 0.67 \% & 6.12 \% & 0.00 \% & 1.98 \% & 2.27 \% & 2.61 \% & 0.00 \% \\ \text { RSA } & 0.00 \% & 5.10 \% & 0.00 \% & 1.98 \% & 1.14 \% & 0.87 \% & 0.00 \%\end{array}$

\section{Medium-term options}

$\begin{array}{lccccccc}\text { NFP } & 3.33 \% & 7.27 \% & 2.13 \% & 0.00 \% & 7.87 \% & 2.04 \% & 12.82 \% \\ \text { CCI } & 0.00 \% & 0.00 \% & 2.13 \% & 1.85 \% & 1.12 \% & 0.00 \% & 0.00 \% \\ \text { CPI } & 0.83 \% & 1.82 \% & 4.26 \% & 1.85 \% & 2.25 \% & 2.04 \% & 0.00 \% \\ \text { DGO } & 0.83 \% & 0.00 \% & 0.00 \% & 0.00 \% & 0.00 \% & 1.02 \% & 0.00 \% \\ \text { FOMC } & 0.83 \% & 1.82 \% & 2.13 \% & 0.00 \% & 0.00 \% & 1.02 \% & 12.82 \% \\ \text { GDP } & 0.83 \% & 5.45 \% & 0.00 \% & 1.85 \% & 1.12 \% & 1.02 \% & 0.00 \% \\ \text { IJC } & 0.83 \% & 3.64 \% & 2.13 \% & 1.85 \% & 3.37 \% & 0.00 \% & 2.56 \% \\ \text { LI } & 0.00 \% & 0.00 \% & 0.00 \% & 0.00 \% & 1.12 \% & 1.02 \% & 0.00 \% \\ \text { NHS } & 0.00 \% & 0.00 \% & 0.00 \% & 1.85 \% & 0.00 \% & 1.02 \% & 2.56 \% \\ \text { PPI } & 1.67 \% & 0.00 \% & 0.00 \% & 0.00 \% & 0.00 \% & 0.00 \% & 0.00 \% \\ \text { RSA } & 0.00 \% & 0.00 \% & 0.00 \% & 0.00 \% & 1.12 \% & 1.02 \% & 0.00 \%\end{array}$

\section{Long-term options}

\begin{tabular}{llllllll} 
NFP & $0.86 \%$ & $2.63 \%$ & $2.33 \%$ & $0.00 \%$ & $4.55 \%$ & $2.91 \%$ & $8.33 \%$ \\
CCI & $2.59 \%$ & $2.63 \%$ & $2.33 \%$ & $0.00 \%$ & $6.82 \%$ & $0.00 \%$ & $5.56 \%$ \\
CPI & $0.00 \%$ & $0.00 \%$ & $2.33 \%$ & $0.00 \%$ & $4.55 \%$ & $0.00 \%$ & $0.00 \%$ \\
DGO & $1.72 \%$ & $0.00 \%$ & $0.00 \%$ & $0.00 \%$ & $0.00 \%$ & $0.97 \%$ & $0.00 \%$ \\
FOMC & $0.00 \%$ & $5.26 \%$ & $4.65 \%$ & $0.00 \%$ & $0.00 \%$ & $2.91 \%$ & $13.89 \%$ \\
GDP & $0.00 \%$ & $0.00 \%$ & $0.00 \%$ & $0.00 \%$ & $0.00 \%$ & $0.91 \%$ & $0.00 \%$ \\
IJC & $0.86 \%$ & $0.00 \%$ & $0.00 \%$ & $0.00 \%$ & $4.55 \%$ & $0.00 \%$ & $2.78 \%$ \\
LI & $0.86 \%$ & $0.00 \%$ & $0.00 \%$ & $0.00 \%$ & $0.00 \%$ & $0.00 \%$ & $0.00 \%$ \\
NHS & $1.72 \%$ & $0.00 \%$ & $4.65 \%$ & $2.70 \%$ & $0.00 \%$ & $0.00 \%$ & $2.78 \%$ \\
PPI & $0.00 \%$ & $2.63 \%$ & $0.00 \%$ & $0.00 \%$ & $0.00 \%$ & $0.00 \%$ & $0.00 \%$ \\
RSA & $0.00 \%$ & $0.00 \%$ & $0.00 \%$ & $0.00 \%$ & $0.00 \%$ & $0.97 \%$ & $0.00 \%$ \\
\hline
\end{tabular}


Table 10: Relation between jumps and scheduled news announcements disaggregated by announcement items (non-crisis and crisis subsamples)

Continued from previous page

\begin{tabular}{ccccccc}
\hline $\begin{array}{c}\text { DOTM } \\
\text { puts }\end{array}$ & $\begin{array}{c}\text { OTM } \\
\text { puts }\end{array}$ & $\begin{array}{c}\text { ATM } \\
\text { puts }\end{array}$ & $\begin{array}{c}\text { ATM } \\
\text { calls }\end{array}$ & $\begin{array}{c}\text { OTM } \\
\text { calls }\end{array}$ & $\begin{array}{c}\text { DOTM } \\
\text { calls }\end{array}$ & Futures \\
\hline
\end{tabular}

Panel B: $P(N e w s \mid J u m p)$ over the crisis subsample

Short-term options

$\begin{array}{lccccccc}\text { NFP } & 9.29 \% & 15.49 \% & 6.84 \% & 10.10 \% & 9.80 \% & 6.04 \% & 12.73 \% \\ \text { CCI } & 0.00 \% & 0.00 \% & 1.71 \% & 3.03 \% & 0.00 \% & 0.55 \% & 0.00 \% \\ \text { CPI } & 2.14 \% & 1.41 \% & 2.56 \% & 2.02 \% & 7.84 \% & 0.55 \% & 0.00 \% \\ \text { DGO } & 2.14 \% & 1.41 \% & 3.42 \% & 4.04 \% & 0.00 \% & 2.75 \% & 0.00 \% \\ \text { FOMC } & 1.43 \% & 2.82 \% & 2.56 \% & 2.02 \% & 5.88 \% & 1.10 \% & 7.27 \% \\ \text { GDP } & 1.43 \% & 4.23 \% & 1.71 \% & 4.04 \% & 5.88 \% & 4.40 \% & 1.82 \% \\ \text { IJC } & 12.86 \% & 2.82 \% & 11.11 \% & 7.07 \% & 13.73 \% & 12.09 \% & 0.00 \% \\ \text { LI } & 0.71 \% & 0.00 \% & 0.85 \% & 1.01 \% & 0.00 \% & 0.55 \% & 0.00 \% \\ \text { NHS } & 0.71 \% & 1.41 \% & 0.00 \% & 1.01 \% & 0.00 \% & 1.10 \% & 0.00 \% \\ \text { PPI } & 2.86 \% & 1.41 \% & 0.00 \% & 1.01 \% & 0.00 \% & 1.10 \% & 0.00 \% \\ \text { RSA } & 2.14 \% & 1.41 \% & 0.00 \% & 0.00 \% & 5.88 \% & 1.10 \% & 0.00 \%\end{array}$

Medium-term options

$\begin{array}{lccccccc}\text { NFP } & 9.35 \% & 9.46 \% & 7.14 \% & 11.54 \% & 13.11 \% & 2.42 \% & 15.09 \% \\ \text { CCI } & 0.00 \% & 1.35 \% & 1.79 \% & 1.92 \% & 0.00 \% & 0.61 \% & 0.00 \% \\ \text { CPI } & 0.93 \% & 0.00 \% & 0.89 \% & 0.00 \% & 0.00 \% & 2.42 \% & 0.00 \% \\ \text { DGO } & 0.00 \% & 2.70 \% & 0.89 \% & 0.00 \% & 6.56 \% & 1.82 \% & 0.00 \% \\ \text { FOMC } & 2.80 \% & 4.05 \% & 2.68 \% & 3.85 \% & 3.28 \% & 0.61 \% & 7.55 \% \\ \text { GDP } & 0.00 \% & 2.70 \% & 0.00 \% & 1.92 \% & 1.64 \% & 0.61 \% & 1.89 \% \\ \text { IJC } & 5.61 \% & 14.86 \% & 1.79 \% & 7.69 \% & 8.20 \% & 9.70 \% & 0.00 \% \\ \text { LI } & 0.93 \% & 1.35 \% & 0.00 \% & 0.00 \% & 0.00 \% & 1.21 \% & 0.00 \% \\ \text { NHS } & 0.00 \% & 0.00 \% & 0.89 \% & 1.92 \% & 1.64 \% & 0.61 \% & 0.00 \% \\ \text { PPI } & 0.93 \% & 1.35 \% & 0.89 \% & 0.00 \% & 0.00 \% & 1.21 \% & 0.00 \% \\ \text { RSA } & 0.93 \% & 2.70 \% & 0.00 \% & 1.92 \% & 0.00 \% & 0.61 \% & 0.00 \%\end{array}$

\section{Long-term options}

\begin{tabular}{llllllll} 
NFP & $5.61 \%$ & $8.82 \%$ & $4.90 \%$ & $11.43 \%$ & $9.46 \%$ & $3.11 \%$ & $12.00 \%$ \\
CCI & $0.00 \%$ & $1.47 \%$ & $1.96 \%$ & $0.00 \%$ & $1.35 \%$ & $0.00 \%$ & $0.00 \%$ \\
CPI & $1.87 \%$ & $0.00 \%$ & $0.98 \%$ & $0.00 \%$ & $6.76 \%$ & $1.55 \%$ & $0.00 \%$ \\
DGO & $3.74 \%$ & $0.00 \%$ & $0.98 \%$ & $0.00 \%$ & $1.35 \%$ & $2.07 \%$ & $0.00 \%$ \\
FOMC & $0.94 \%$ & $2.94 \%$ & $2.94 \%$ & $2.86 \%$ & $2.70 \%$ & $0.52 \%$ & $8.00 \%$ \\
GDP & $1.87 \%$ & $1.47 \%$ & $0.00 \%$ & $2.86 \%$ & $5.41 \%$ & $2.07 \%$ & $2.00 \%$ \\
IJC & $7.48 \%$ & $8.82 \%$ & $0.98 \%$ & $5.71 \%$ & $10.82 \%$ & $5.70 \%$ & $0.00 \%$ \\
LI & $1.87 \%$ & $0.00 \%$ & $0.00 \%$ & $0.00 \%$ & $0.00 \%$ & $1.03 \%$ & $0.00 \%$ \\
NHS & $0.00 \%$ & $1.47 \%$ & $0.98 \%$ & $0.00 \%$ & $0.00 \%$ & $0.52 \%$ & $0.00 \%$ \\
PPI & $0.94 \%$ & $0.00 \%$ & $0.00 \%$ & $0.00 \%$ & $1.35 \%$ & $2.07 \%$ & $0.00 \%$ \\
RSA & $0.94 \%$ & $1.47 \%$ & $0.00 \%$ & $11.43 \%$ & $0.00 \%$ & $3.11 \%$ & $0.00 \%$ \\
\hline
\end{tabular}


Table 10: Relation between jumps and scheduled news announcements disaggregated by announcement items (non-crisis and crisis subsamples)

Continued from previous page

\begin{tabular}{ccccccc}
\hline $\begin{array}{c}\text { DOTM } \\
\text { puts }\end{array}$ & $\begin{array}{c}\text { OTM } \\
\text { puts }\end{array}$ & $\begin{array}{c}\text { ATM } \\
\text { puts }\end{array}$ & $\begin{array}{c}\text { ATM } \\
\text { calls }\end{array}$ & $\begin{array}{c}\text { OTM } \\
\text { calls }\end{array}$ & $\begin{array}{c}\text { DOTM } \\
\text { calls }\end{array}$ & Futures \\
\hline
\end{tabular}

Panel C: $P(J u m p \mid N e w s)$ over the non-crisis subsample

\section{Short-term options}

$\begin{array}{lccccccc}\text { NFP } & 6.45 \% & 12.90 \% & 9.68 \% & 6.45 \% & 6.45 \% & 6.45 \% & 12.90 \% \\ \text { CCI } & 3.33 \% & 0.00 \% & 3.33 \% & 0.00 \% & 0.00 \% & 10.00 \% & 0.00 \% \\ \text { CPI } & 12.90 \% & 9.68 \% & 6.45 \% & 16.13 \% & 6.45 \% & 3.23 \% & 0.00 \% \\ \text { DGO } & 9.68 \% & 0.00 \% & 3.23 \% & 0.00 \% & 6.45 \% & 3.23 \% & 0.00 \% \\ \text { FOMC } & 0.00 \% & 0.00 \% & 5.00 \% & 5.00 \% & 0.00 \% & 0.00 \% & 25.00 \% \\ \text { GDP } & 12.90 \% & 0.00 \% & 3.23 \% & 9.68 \% & 3.23 \% & 0.00 \% & 0.00 \% \\ \text { IJC } & 3.73 \% & 5.97 \% & 0.00 \% & 8.96 \% & 6.72 \% & 2.99 \% & 0.75 \% \\ \text { LI } & 0.00 \% & 0.00 \% & 0.00 \% & 0.00 \% & 0.00 \% & 0.00 \% & 0.00 \% \\ \text { NHS } & 0.00 \% & 0.00 \% & 0.00 \% & 3.23 \% & 0.00 \% & 0.00 \% & 3.23 \% \\ \text { PPI } & 3.23 \% & 19.35 \% & 0.00 \% & 6.45 \% & 6.45 \% & 9.68 \% & 0.00 \% \\ \text { RSA } & 0.00 \% & 16.13 \% & 0.00 \% & 6.45 \% & 3.23 \% & 3.23 \% & 0.00 \%\end{array}$

\section{Medium-term options}

$\begin{array}{lccccccc}\text { NFP } & 12.90 \% & 12.90 \% & 3.23 \% & 0.00 \% & 22.58 \% & 6.45 \% & 16.13 \% \\ \text { CCI } & 0.00 \% & 0.00 \% & 3.33 \% & 3.33 \% & 3.33 \% & 0.00 \% & 0.00 \% \\ \text { CPI } & 3.23 \% & 3.23 \% & 6.45 \% & 3.23 \% & 6.45 \% & 6.45 \% & 0.00 \% \\ \text { DGO } & 3.23 \% & 0.00 \% & 0.00 \% & 0.00 \% & 0.00 \% & 3.23 \% & 0.00 \% \\ \text { FOMC } & 5.00 \% & 5.00 \% & 5.00 \% & 0.00 \% & 0.00 \% & 5.00 \% & 25.00 \% \\ \text { GDP } & 3.23 \% & 9.68 \% & 0.00 \% & 3.23 \% & 3.23 \% & 3.23 \% & 0.00 \% \\ \text { IJC } & 0.75 \% & 1.49 \% & 0.75 \% & 0.75 \% & 2.24 \% & 0.00 \% & 0.75 \% \\ \text { LI } & 0.00 \% & 0.00 \% & 0.00 \% & 0.00 \% & 3.23 \% & 3.23 \% & 0.00 \% \\ \text { NHS } & 0.00 \% & 0.00 \% & 0.00 \% & 3.23 \% & 0.00 \% & 3.23 \% & 3.23 \% \\ \text { PPI } & 6.45 \% & 0.00 \% & 0.00 \% & 0.00 \% & 0.00 \% & 0.00 \% & 0.00 \% \\ \text { RSA } & 0.00 \% & 0.00 \% & 0.00 \% & 0.00 \% & 3.23 \% & 3.23 \% & 0.00 \%\end{array}$

\section{Long-term options}

\begin{tabular}{lccccccc} 
NFP & $3.23 \%$ & $3.23 \%$ & $3.23 \%$ & $0.00 \%$ & $6.45 \%$ & $9.68 \%$ & $9.68 \%$ \\
CCI & $10.00 \%$ & $3.33 \%$ & $3.33 \%$ & $0.00 \%$ & $10.00 \%$ & $0.00 \%$ & $6.67 \%$ \\
CPI & $0.00 \%$ & $0.00 \%$ & $3.23 \%$ & $0.00 \%$ & $6.45 \%$ & $0.00 \%$ & $0.00 \%$ \\
DGO & $6.45 \%$ & $0.00 \%$ & $0.00 \%$ & $0.00 \%$ & $0.00 \%$ & $3.23 \%$ & $0.00 \%$ \\
FOMC & $0.00 \%$ & $10.00 \%$ & $10.00 \%$ & $0.00 \%$ & $0.00 \%$ & $15.00 \%$ & $25.00 \%$ \\
GDP & $0.00 \%$ & $0.00 \%$ & $0.00 \%$ & $0.00 \%$ & $0.00 \%$ & $3.23 \%$ & $0.00 \%$ \\
IJC & $0.75 \%$ & $0.00 \%$ & $0.00 \%$ & $0.00 \%$ & $1.49 \%$ & $0.00 \%$ & $0.75 \%$ \\
LI & $3.23 \%$ & $0.00 \%$ & $0.00 \%$ & $0.00 \%$ & $0.00 \%$ & $0.00 \%$ & $0.00 \%$ \\
NHS & $6.45 \%$ & $0.00 \%$ & $6.45 \%$ & $3.23 \%$ & $0.00 \%$ & $0.00 \%$ & $3.23 \%$ \\
PPI & $0.00 \%$ & $3.23 \%$ & $0.00 \%$ & $0.00 \%$ & $0.00 \%$ & $0.00 \%$ & $0.00 \%$ \\
RSA & $0.00 \%$ & $0.00 \%$ & $0.00 \%$ & $0.00 \%$ & $0.00 \%$ & $3.23 \%$ & $0.00 \%$ \\
\hline
\end{tabular}


Table 10: Relation between jumps and scheduled news announcements disaggregated by announcement items (non-crisis and crisis subsamples)

Continued from previous page

\begin{tabular}{ccccccc}
$\begin{array}{c}\text { DOTM } \\
\text { puts }\end{array}$ & $\begin{array}{c}\text { OTM } \\
\text { puts }\end{array}$ & $\begin{array}{c}\text { ATM } \\
\text { puts }\end{array}$ & $\begin{array}{c}\text { ATM } \\
\text { calls }\end{array}$ & $\begin{array}{c}\text { OTM } \\
\text { calls }\end{array}$ & $\begin{array}{c}\text { DOTM } \\
\text { calls }\end{array}$ & Futures \\
\hline Panel D: $P($ Jump $\mid$ News $)$ & over the crisis subsample & &
\end{tabular}

Panel D: P(Jump $\mid N$

$\begin{array}{lccccccc}\text { NFP } & 31.71 \% & 26.83 \% & 19.51 \% & 24.39 \% & 12.20 \% & 26.83 \% & 17.07 \% \\ \text { CCI } & 0.00 \% & 0.00 \% & 4.88 \% & 7.32 \% & 0.00 \% & 2.44 \% & 0.00 \% \\ \text { CPI } & 7.32 \% & 2.44 \% & 7.32 \% & 4.88 \% & 9.76 \% & 2.44 \% & 0.00 \% \\ \text { DGO } & 7.32 \% & 2.44 \% & 9.76 \% & 9.76 \% & 0.00 \% & 12.20 \% & 0.00 \% \\ \text { FOMC } & 6.67 \% & 6.67 \% & 10.00 \% & 6.67 \% & 10.00 \% & 6.67 \% & 13.33 \% \\ \text { GDP } & 4.88 \% & 7.32 \% & 4.88 \% & 9.76 \% & 7.32 \% & 19.51 \% & 2.44 \% \\ \text { IJC } & 10.06 \% & 1.12 \% & 7.26 \% & 3.91 \% & 3.91 \% & 12.29 \% & 0.00 \% \\ \text { LI } & 2.44 \% & 0.00 \% & 2.44 \% & 2.44 \% & 0.00 \% & 2.44 \% & 0.00 \% \\ \text { NHS } & 2.44 \% & 2.44 \% & 0.00 \% & 2.44 \% & 0.00 \% & 4.88 \% & 0.00 \% \\ \text { PPI } & 9.76 \% & 2.44 \% & 0.00 \% & 2.44 \% & 0.00 \% & 4.88 \% & 0.00 \% \\ \text { RSA } & 7.32 \% & 2.44 \% & 0.00 \% & 0.00 \% & 7.32 \% & 4.88 \% & 0.00 \%\end{array}$

\section{Medium-term options}

$\begin{array}{lccccccc}\text { NFP } & 24.39 \% & 17.07 \% & 19.51 \% & 14.63 \% & 19.51 \% & 9.76 \% & 19.51 \% \\ \text { CCI } & 0.00 \% & 2.44 \% & 4.88 \% & 2.44 \% & 0.00 \% & 2.44 \% & 0.00 \% \\ \text { CPI } & 2.44 \% & 0.00 \% & 2.44 \% & 0.00 \% & 0.00 \% & 9.76 \% & 0.00 \% \\ \text { DGO } & 0.00 \% & 4.88 \% & 2.44 \% & 0.00 \% & 9.76 \% & 7.32 \% & 0.00 \% \\ \text { FOMC } & 10.00 \% & 10.00 \% & 10.00 \% & 6.67 \% & 6.67 \% & 3.33 \% & 13.33 \% \\ \text { GDP } & 0.00 \% & 4.88 \% & 0.00 \% & 2.44 \% & 2.44 \% & 2.44 \% & 2.44 \% \\ \text { IJC } & 3.35 \% & 6.15 \% & 1.12 \% & 2.23 \% & 2.79 \% & 8.94 \% & 0.00 \% \\ \text { LI } & 2.44 \% & 2.44 \% & 0.00 \% & 0.00 \% & 0.00 \% & 4.88 \% & 0.00 \% \\ \text { NHS } & 0.00 \% & 0.00 \% & 2.44 \% & 2.44 \% & 2.44 \% & 2.44 \% & 0.00 \% \\ \text { PPI } & 2.44 \% & 2.44 \% & 2.44 \% & 0.00 \% & 0.00 \% & 4.88 \% & 0.00 \% \\ \text { RSA } & 2.44 \% & 4.88 \% & 0.00 \% & 2.44 \% & 0.00 \% & 2.44 \% & 0.00 \%\end{array}$

\section{Long-term options}

$\begin{array}{lccccccc}\text { NFP } & 14.63 \% & 14.63 \% & 12.20 \% & 9.76 \% & 17.07 \% & 14.63 \% & 14.63 \% \\ \text { CCI } & 0.00 \% & 2.44 \% & 4.88 \% & 0.00 \% & 2.44 \% & 0.00 \% & 0.00 \% \\ \text { CPI } & 4.88 \% & 0.00 \% & 2.44 \% & 0.00 \% & 12.20 \% & 7.32 \% & 0.00 \% \\ \text { DGO } & 9.76 \% & 0.00 \% & 2.44 \% & 0.00 \% & 2.44 \% & 9.76 \% & 0.00 \% \\ \text { FOMC } & 3.33 \% & 6.67 \% & 10.00 \% & 3.33 \% & 6.67 \% & 3.33 \% & 13.33 \% \\ \text { GDP } & 4.88 \% & 2.44 \% & 0.00 \% & 2.44 \% & 9.76 \% & 9.76 \% & 2.44 \% \\ \text { IJC } & 4.47 \% & 3.35 \% & 0.56 \% & 1.12 \% & 4.47 \% & 6.15 \% & 0.00 \% \\ \text { LI } & 4.88 \% & 0.00 \% & 0.00 \% & 0.00 \% & 0.00 \% & 4.88 \% & 0.00 \% \\ \text { NHS } & 0.00 \% & 2.44 \% & 2.44 \% & 0.00 \% & 0.00 \% & 2.44 \% & 0.00 \% \\ \text { PPI } & 2.44 \% & 0.00 \% & 0.00 \% & 0.00 \% & 2.44 \% & 9.76 \% & 0.00 \% \\ \text { RSA } & 2.44 \% & 2.44 \% & 0.00 \% & 9.76 \% & 0.00 \% & 14.63 \% & 0.00 \%\end{array}$

Entries report summary statistics on the relation between detected jumps and macroeconomic news announcements disaggregated by news items for all investigated delta and maturity categories over the non-crisis subsample (Panels A and C) and the crisis subsample (Panels B and D). The probability of a jump to be related to a specific news announcement $P(N e w s \mid J u m p)$ (Panels A and B) and the probability of a specific news announcement leading to a jump $P($ Jump $\mid$ News) (Panels C and D) are reported. Jumps have been detected using the Lee and Mykland (2008) jump detection methodology based on a significance level of $\alpha=0.1 \%$. Jumps are defined to be related to a news announcement if they occurred within \pm 10 minutes of an announcement. The sample period is January 1, 2005 to July 31, 2007 for the non-crisis subsample and August 1, 2007 to December 31, 2010 for the crisis subsample. 
Table 11: Information shocks, volume, and illiquidity as jump determinants (crisis subsample)

\begin{tabular}{|c|c|c|c|}
\hline & Short-term & Medium-term & Long-term \\
\hline$c$ & $-3.2964^{* * *}$ & $-3.4553^{* * *}$ & $-3.9756^{* * *}$ \\
\hline$s B A_{t-1}$ & $0.0706^{*}$ & $0.132^{* * *}$ & $0.1571^{* * *}$ \\
\hline BidSize $_{t-1}$ & -0.0017 & -0.0019 & -0.0005 \\
\hline AskSize $e_{t-1}$ & -0.0005 & -0.0032 & $-0.0089^{* *}$ \\
\hline Volume $_{t-1, t}$ & -0.0053 & $0.0035^{*}$ & 0.0017 \\
\hline$N F P_{t}$ & $0.5986^{* * *}$ & 0.2969 & -0.0498 \\
\hline$C C I_{t}$ & -1.7225 & -10.3653 & - \\
\hline$C P I_{t}$ & -0.1908 & -1.0597 & 0.0343 \\
\hline$D G O_{t}$ & -0.1901 & -0.9037 & -1.4842 \\
\hline$F O M C_{t}$ & - & - & - \\
\hline$G D P_{t}$ & 0.0403 & -1.5392 & $0.6491^{* * *}$ \\
\hline$I J C_{t}$ & -0.0839 & -0.1693 & 0.036 \\
\hline$L I_{t}$ & -0.0886 & -0.3529 & - \\
\hline$N H S_{t}$ & - & -4.8425 & - \\
\hline$P P I_{t}$ & -0.3548 & -1.9787 & - \\
\hline$R S A_{t}$ & -0.3868 & -0.8008 & 0.485 \\
\hline
\end{tabular}

Entries report the estimation results for the logistic regression model in equation (11) over the crisis subsample. The estimation is performed separately for short-, medium-, and long-term options on a sample pooled across all delta categories. Only news-related observations are considered. The estimation is performed via maximum likelihood and $* * *, * *$, and $*$ report statistical significance at the 1\%,5\%, and 10\% levels, respectively. The sample period is from August 1, 2007 to December 31, 2010 . 
Table 12: Relation between jumps and unscheduled news announcements

\begin{tabular}{|c|c|c|c|c|c|c|c|}
\hline & $\begin{array}{c}\text { DOTM } \\
\text { puts }\end{array}$ & $\begin{array}{c}\text { OTM } \\
\text { puts }\end{array}$ & $\begin{array}{l}\text { ATM } \\
\text { puts }\end{array}$ & $\begin{array}{l}\text { ATM } \\
\text { calls }\end{array}$ & $\begin{array}{l}\text { OTM } \\
\text { calls }\end{array}$ & $\begin{array}{c}\text { DOTM } \\
\text { calls }\end{array}$ & Futures \\
\hline $\begin{array}{l}\text { Short-term options } \\
\text { \# Jump days equal to } \\
\text { unsched. news day } \\
\% \text { Jump days equal to } \\
\text { \% Unsched. news day }\end{array}$ & $\begin{array}{c}5 \\
2.16 \%\end{array}$ & $\begin{array}{c}4 \\
2.99 \%\end{array}$ & $\begin{array}{c}3 \\
2.34 \%\end{array}$ & $\begin{array}{c}2 \\
1.24 \%\end{array}$ & $\begin{array}{c}0 \\
0.00 \%\end{array}$ & $\begin{array}{c}10 \\
4.07 \%\end{array}$ & $\begin{array}{c}3 \\
4.17 \%\end{array}$ \\
\hline $\begin{array}{l}\text { Medium-term optior } \\
\text { \# Jump days equal to } \\
\text { unsched. news day } \\
\% \text { Jump days equal to } \\
\text { unsched. news day }\end{array}$ & $\begin{array}{c}3 \\
1.66 \%\end{array}$ & $\begin{array}{c}4 \\
4.00 \%\end{array}$ & $6.31 \%$ & $\begin{array}{c}3 \\
3.75 \%\end{array}$ & $\begin{array}{c}3 \\
2.40 \%\end{array}$ & $\begin{array}{c}7 \\
3.21 \%\end{array}$ & $\begin{array}{c}3 \\
4.29 \%\end{array}$ \\
\hline $\begin{array}{l}\text { Long-term options } \\
\text { \# Jump days equal to } \\
\text { unsched. news day } \\
\% \text { Jump days equal to } \\
\text { unsched. news day }\end{array}$ & $2.52 \%$ & $5.00 \%$ & $\begin{array}{c}7 \\
6.25 \%\end{array}$ & $1.61 \%$ & $1.03 \%$ & $4.05 \%$ & $4.48 \%$ \\
\hline
\end{tabular}

Entries report summary statistics on the relation between detected jumps and unscheduled news announcements for all investigated delta and maturity categories (Panels A to C). The number of jump days that are equal to a day on which unscheduled news has been released and this number as a fraction of all jump days is reported. Jumps have been detected using the Lee and Mykland (2008) jump detection methodology based on a significance level of $\alpha=0.1 \%$. The sample period is from January 1, 2005 to December 31, 2010. 
Figure 1: Volatility signature plots of short-term options returns
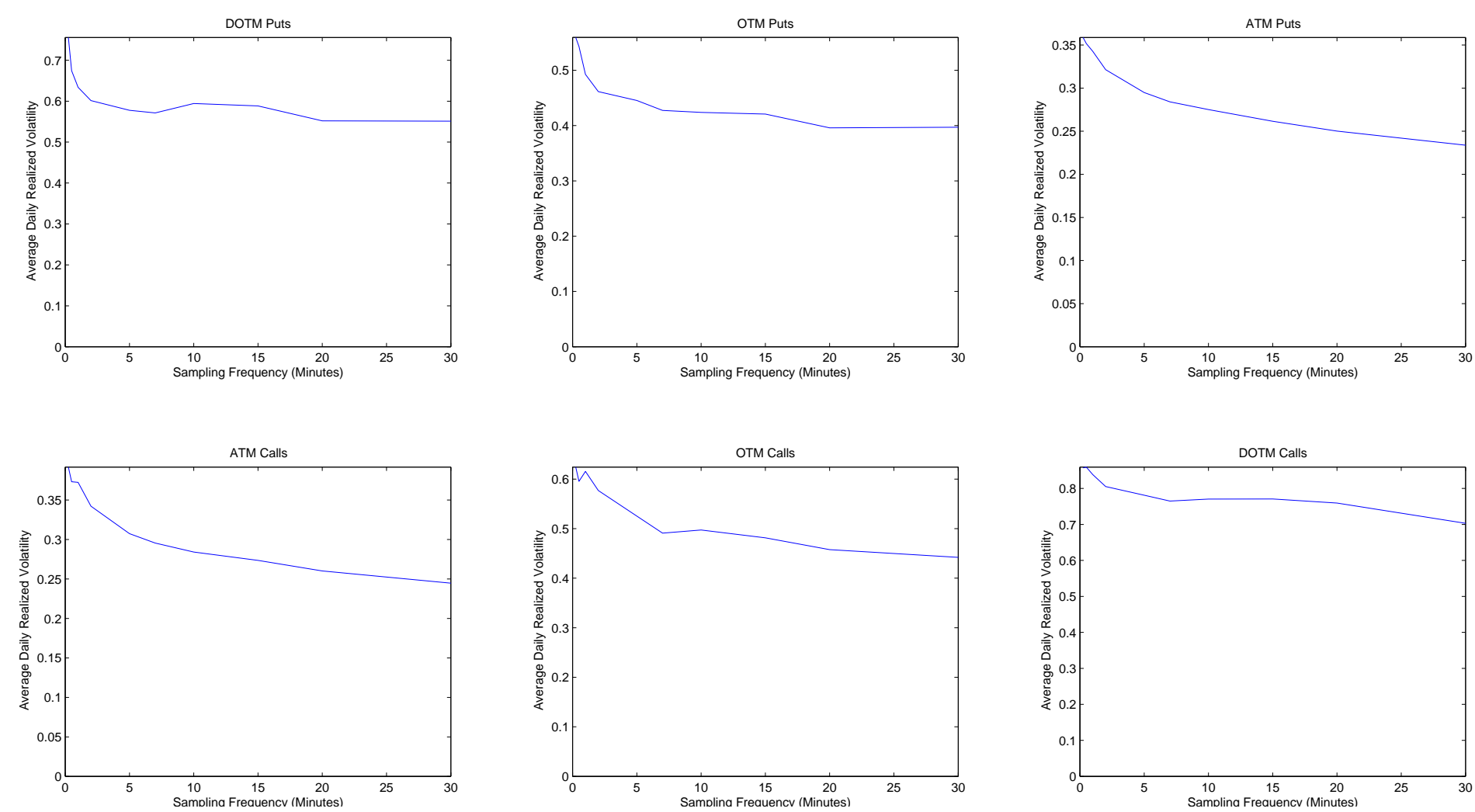

The figure depicts the average daily realized volatility of option returns as a function of the sampling frequency for short-term options of different delta categories. We consider the following delta categories (defined in Table 1) for calls and puts: deep out-of-the-money (DOTM), out-of-the-money (OTM), and at-the-money (ATM). 
Figure 2: Distribution of Co-Jumps

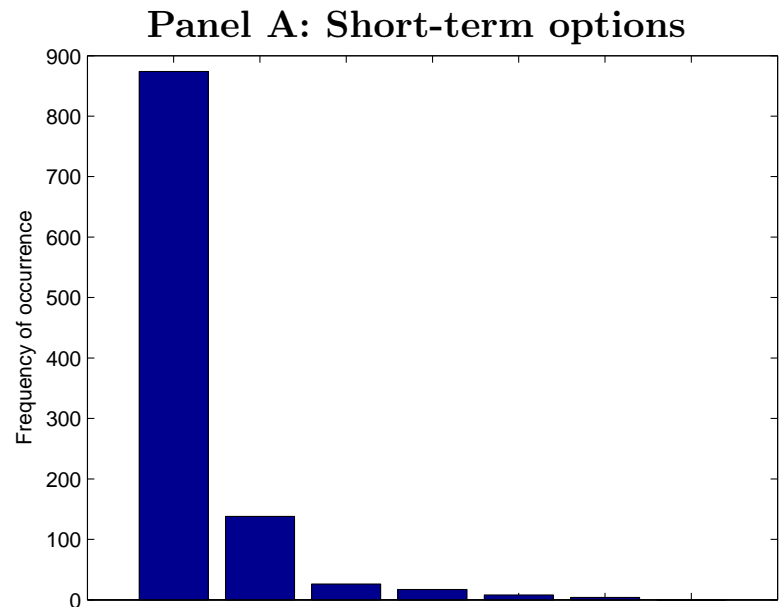

Panel B: Medium-term options

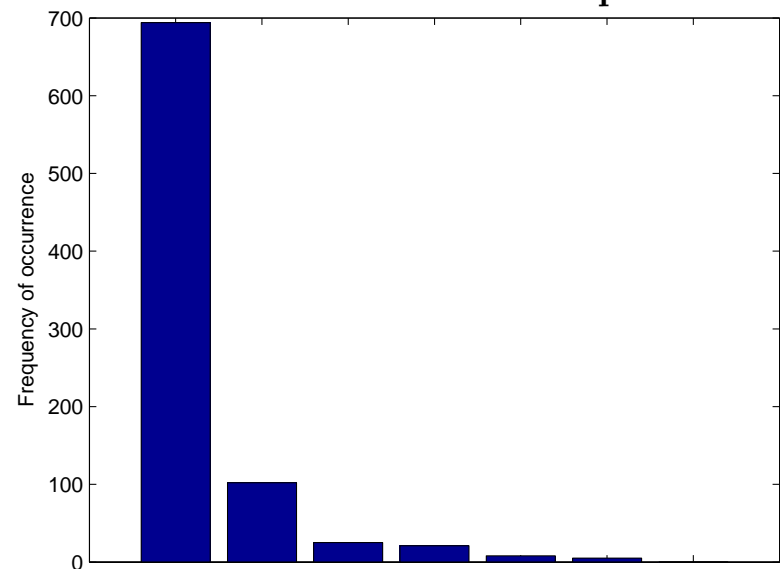

Panel C: Long-term options

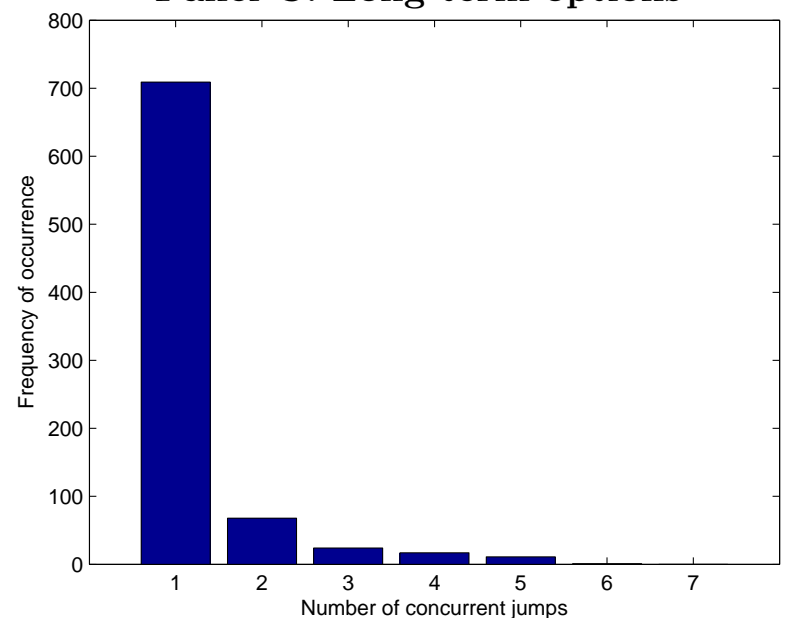

The figure illustrates the distribution of co-jump events for short-, medium-, and long-term options (Panels A, B, and C, respectively). Co-jump events are defined by the number of concurrent jumps across different delta levels and the underlying asset. The event of only one concurrent jump corresponds to an idiosyncratic jump in only one of the delta categories or the underlying asset. The frequency of occurrence is reported for each possible co-jump event. 
Figure 3: Composition of co-jumps

Panel A: Short-term options

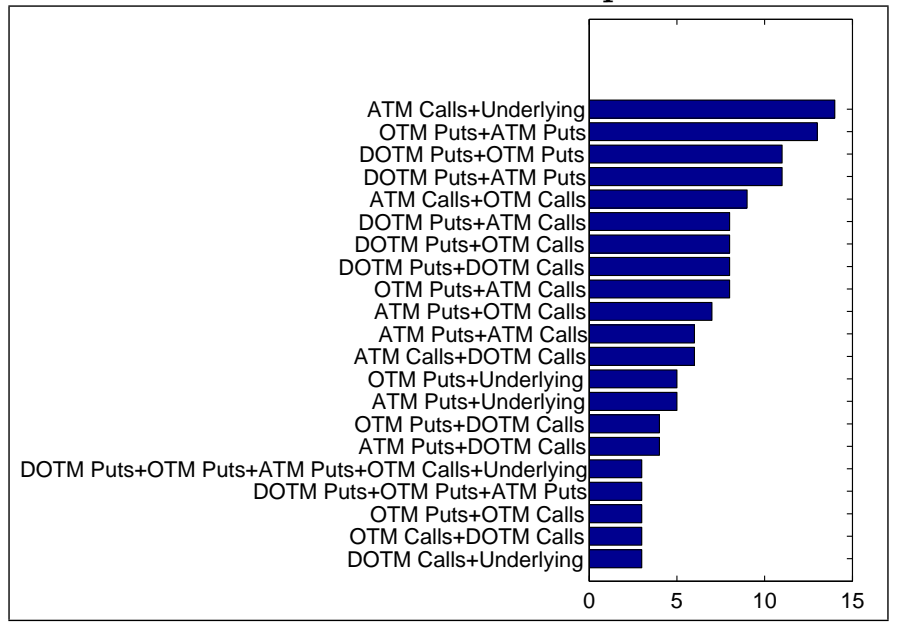

Panel B: Medium-term options

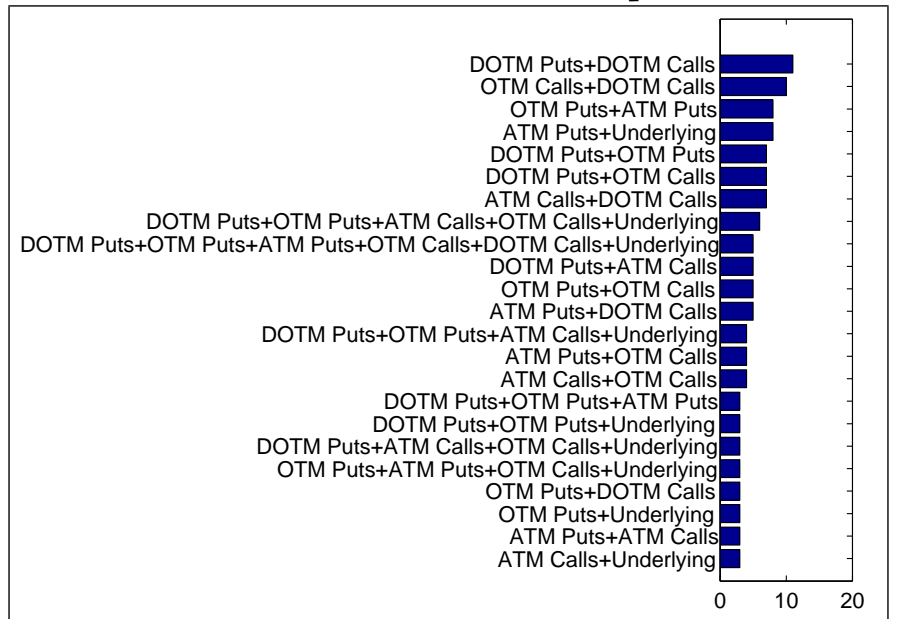

Panel C: Long-term options

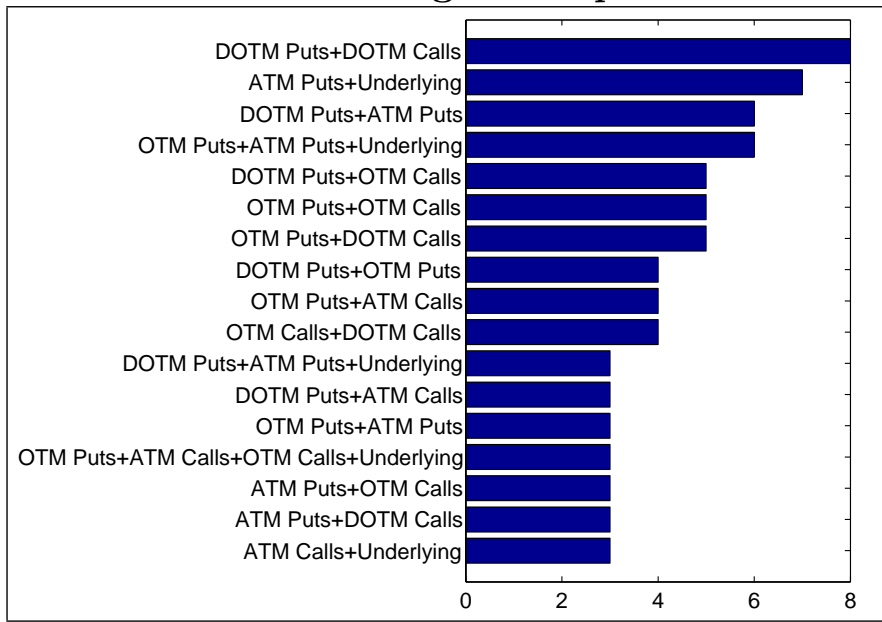

The figure illustrates the composition of the most frequent co-jump events for short-, medium-, and long-term options (Panels A, B, and C, respectively). The composition of a co-jump event is characterized by the delta categories of the options and/or the underlying asset that simultaneously exhibit a jump. 
Figure 4: Option bid-ask spreads around jumps
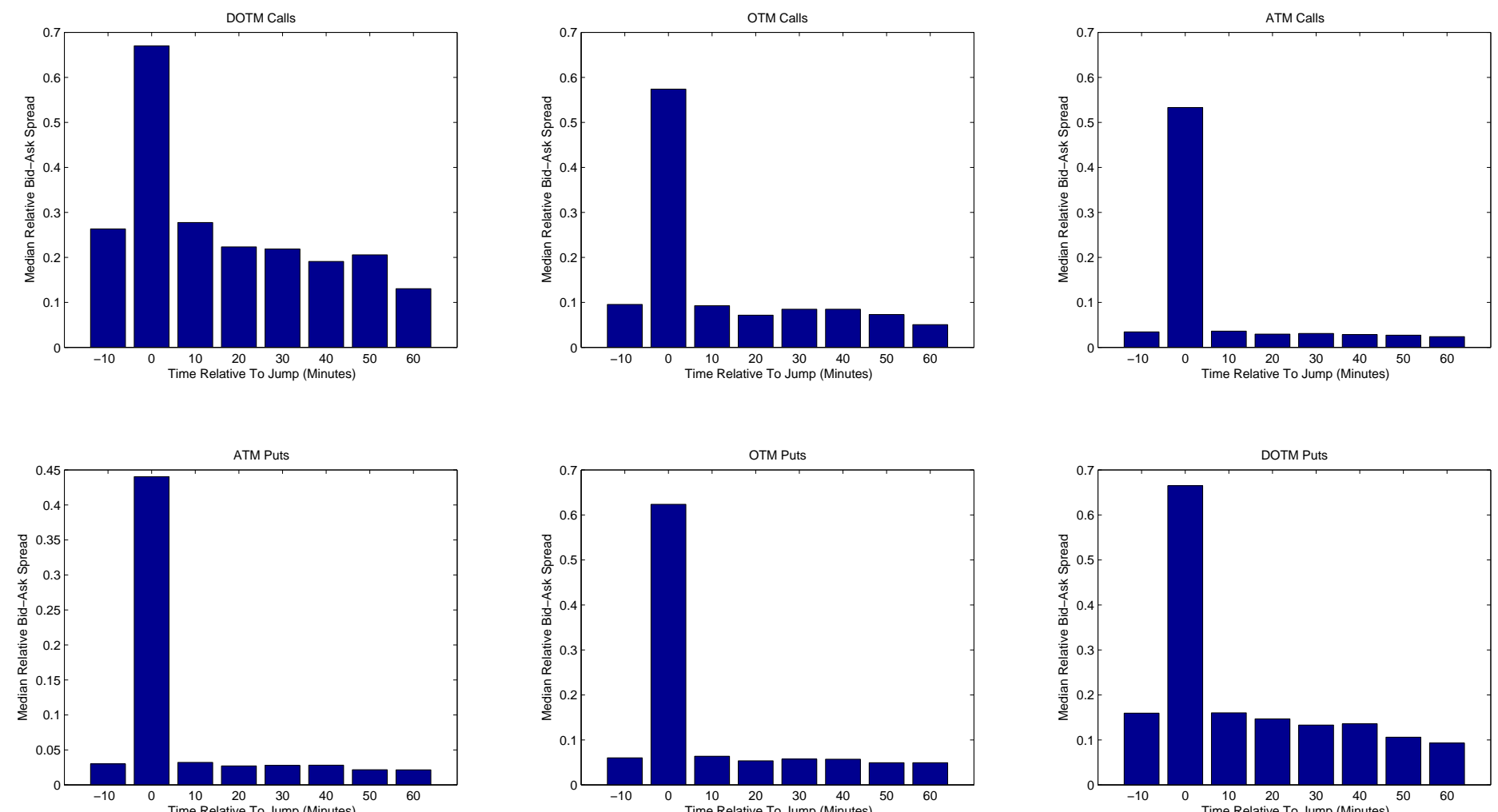

The figure illustrates the median relative option bid-ask spreads for a number of time subintervals around the news-related jumps (10 minutes before the jump up to 60 minutes after the jump) across the various moneyness levels for the case of the short maturity options. We consider deep out-of-the-money (DOTM), out-of-the-money (OTM), and at-the-money (ATM) calls and puts (defined in Table 1). The jump time corresponds to point zero in the graph. 
Figure 5: Distribution of option trading volume before news-related price jumps of short-term options
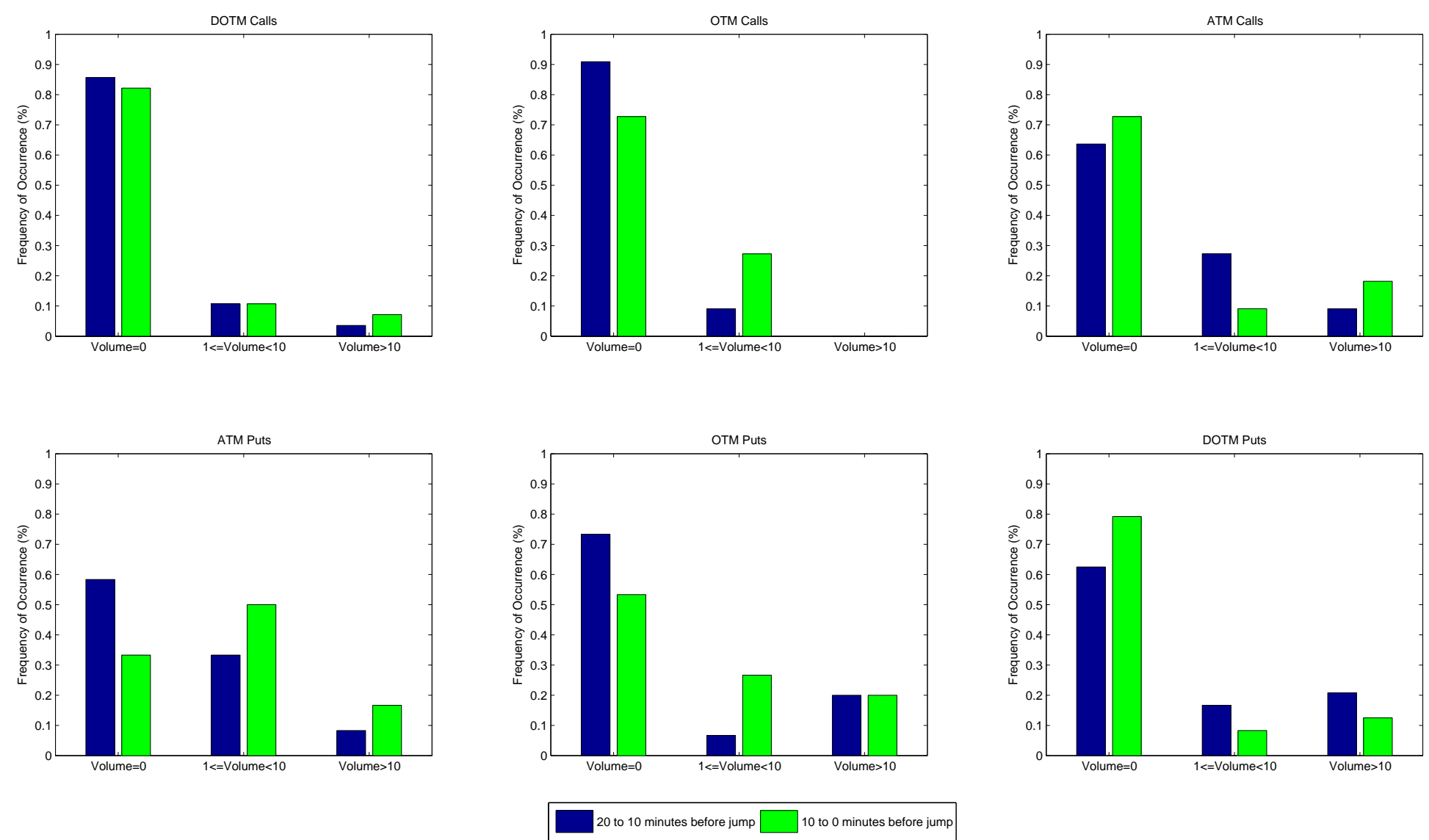

The figure illustrates the frequency (in \%) distribution in the two 10-minutes subintervals before news-related option price jumps of short-term options. The frequency distribution is constructed for option trading volume buckets of zero, 10 or more than 10 contracts. We consider deep out-of-the-money (DOTM), out-of-the-money (OTM), and at-the-money (ATM) calls and puts (defined in Table 1). 
Figure 6: Dynamics of short-term options' bid-ask spreads


The figure illustrates the time evolution of the daily average relative bid-ask spread of short-term options of different delta categories [out-of-themoney (DOTM), out-of-the-money (OTM), and at-the-money (ATM) calls and puts as defined in Table 1] over the non-crisis and crisis subsamples. The dashed line illustrates the non-crisis/crisis split point. 


\title{
Online Appendix to
}

\section{"Jumps in option prices and their determinants: Real-time evidence from the E-mini S\&P 500 options market"}

\author{
George Kapetanios? Eirini Konstantinidi, \\ Michael Neumann, George Skiadopoulos ${ }^{c}$
}

August 12, 2019

In this Online Appendix, we provide additional results obtained from the analysis on E-mini index options (Sections 1-4), as well as results obtained from the analysis on the S\&P 500 index options market (Section 5).

\section{Volatility signature plots}

Following Andersen et al. (2000), we employ volatility signature plots of high-frequency E-mini option returns to select the "optimal" subinterval length for the calculation of the returns. Volatility signature plots depict realized volatility as a function of the sampling frequency. In the absence of microstructure noise, realized volatility, defined as the squared root of summed squared intraday returns, should be invariant to changes in the sampling frequency provided the data is sampled fine enough. Panels A, B, and C of Figure OA.1 show the volatility signature plots for the short-, medium-, and long-term options, respectively. For each maturity category, we consider deep outof-the-money (DOTM), out-of-the-money (OTM), and at-the-money (ATM) calls and puts, as

\footnotetext{
§King's Business School, King's College London, UK, george.kapetanios@kcl.ac.uk

${ }^{a}$ Alliance Manchester Business School, University of Manchester, UK, eirini.konstantinidi@manchester.ac.uk

${ }^{b}$ Independent, michael.neumann.ln@gmail.com

${ }^{c}$ Corresponding author. School of Economics and Finance, Queen Mary, University of London, UK, and Department of Banking and Financial Management, University of Piraeus, Greece. Also Associate Research Fellow with Cass Business School and Warwick Business School, g.skiadopoulos@qmul.ac.uk, gskiado@unipi.gr
} 
defined in Table 1 in the main paper. We can see that the realized volatilities start converging around the 10-minute mark. Hence, we choose a subinterval length of $\Delta t=10$ minutes.

\section{Broadening the definition of concurrent jumps}

In our main analysis, we define simultaneous jumps in E-mini option prices as jumps occurring within the same 10-minute interval. We investigate further the simultaneity of jumps across delta and maturities categories by considering the adjacent 10-minute intervals. Focusing on each maturity and delta bucket separately, we calculate the number of jumps that the adjacent categories exhibit 10 minutes before, simultaneously, and 10 minutes after we detect a jump in the bucket under consideration.

Figure OA.2 shows the number of co-jumps occurring in the three 10-minute intervals [i.e., $(-10,0,+10)$ with zero corresponding to the jump time in any given bucket]. Panels $\mathrm{A}, \mathrm{B}$, and C show the results for the short-, medium-, and long-term contracts, respectively. We can see that in the vast majority of the cases when we detect a jump in a specific bucket at time $t=0$, we do not detect any jumps 10 minutes before $(t=-10)$, simultaneously $(t=0)$ or 10 minutes after $(t=+10)$ in the remaining categories. This corroborates further that the detected jumps are predominantly idiosyncratic.

\section{Volume as a jump driver}

We plot the distribution of trading volume of E-mini options on the jump time, as well as ten minutes before the jump, to check whether option jumps are driven by pre-announcement private information. Panels A, B, and C of Figure OA.3 plot the volume distribution for any given delta bucket for the short-, medium-, and long-term maturity options, respectively. We show the frequency (in \%) distribution of three volume buckets, which correspond to option volume of zero, ten, and more than ten contracts, respectively. We can see that the vast majority of news related jumps is accompanied by zero volume. 


\section{Dynamics of relative bid-ask spread}

As a further robustness check, we examine the dynamics of the illiquidity of the E-mini option market over two subsamples (i.e., non-crisis and crisis periods). Given that the likelihood of option price jumps has been found to be similar across the crisis and non-crisis subsamples, one would expect the dynamics of option market illiquidity not to differ either. This is because our results over the full sample period suggest that the arrival of option price jumps is mostly driven by the illiquidity of the option market.

Panels A, B, and C of Figure OA.4 depict the evolution of the daily average relative bid-ask spread for the various delta categories of short-, medium-, and long-term options, respectively. The dynamics of the option market illiquidity are comparable in the non-crisis and crisis periods, since they do not appear to be any more erratic in the crisis subsample than in the non-crisis subsample. This is in line with the previous finding that option price jumps are equally likely in the non-crisis and crisis periods, provided option price jumps are driven by option market illiquidity. These results further confirm that it is liquidity, and not the content of news shocks, that drives jumps in option prices.

\section{The case of S\&P 500 index options}

We extend our analysis by considering S\&P 500 index options which are actively traded in the Chicago Board of Options Exchange (CBOE). We obtain OPRA trade and quote data on S\&P 500 index options from TickData over the period January 1, 2005 to December 31, 2010. We sample quotes from 9:30 a.m. to 3:45 p.m. Eastern Standard Time (EST), i.e., at times beyond 8:30 a.m. EST, where most of the scheduled macroeconomic news announcements occur; over our sample period, the S\&P 500 options trade from 9:30 a.m. to 4:15 p.m. EST (Regular Trading Hours, RTH). ${ }^{1}$

We apply the same filters as the ones used in our main analysis, to remove noisy data and minimize the effect of microstructure noise (Barndorff-Nielsen et al., 2009). Next, we group options into the maturity and delta buckets shown in Table 1 in the main paper, resulting in

\footnotetext{
${ }^{1} \mathrm{CBOE}$ introduced Extended Trading Hours (ETH) by activating a trading sessions from 2:00 a.m. to 8:15 a.m. in March 2015 which is after the end of our sample period. Even after the introduction of ETH, the trading volume over the ETH is lower than over RTH (e.g.,Bloomberg Tradebook, 2015; Stoev, 2017).
} 
18 different groups (three maturity and six delta groups). To allocate each intraday observation to a delta bucket, we estimate implied volatility and delta using the Black (1976) model. We consider the mid-quote of the shortest E-mini S\&P 500 futures contract as the underlying asset price; this is different from the underlying asset price of E-mini options where we use the midquote of the E-mini futures contract with the same or nearest maturity to the option contract. To avoid non-synchronicity concerns, we match the shortest E-mini S\&P 500 futures quotes to simultaneous S\&P 500 index option quotes. We also proxy intraday interest rates with the daily linearly interpolated U.S. LIBOR rates (LIBOR maturities between one week and one year).

Once we group the option data, we calculate 10-minute returns for the underlying shortest Emini S\&P 500 futures contract and for each option contract of the 18 options buckets. We obtain the 10-minute option returns using quotes for the same contract over two consecutive 10-minute intervals. In the first 10-minute interval, we select the contract that is closest to the bucket's mid-delta point and we track this contract over the second 10-minute interval. If there are more than one quotes satisfying our criteria in any of the two 10-minute intervals, we select the one with a time-stamp closest to the end of the interval. The resulting return is time-stamped at the end of the second 10-minute interval. Given that the majority of the news announcements occurs at 8:30 a.m. EST (i.e., before the S\&P 500 index option market opens), we also calculate overnight returns using quotes from the last 10-minute interval of the previous day and the first 10-minute interval of the current day.

\subsection{Jumps in S\&P 500 index option prices}

We employ the Lee and Mykland's (LM, 2008) test to test if there are any jumps in option prices. Table OA.1 reports summary statistics for the identified jumps. We can see that S\&P 500 index option prices jump. The number of jumps varies across maturity and delta buckets with short maturity options exhibiting the largest number of jumps and largest average size of jumps in absolute terms for any given delta bucket. The probability of a jump occurring ranges between $0.44 \%$ and $1.22 \%$ across maturity and delta buckets. In the case of the underlying price, the number and size of jumps is lower than the number and size of jumps in S\&P 500 index option prices.

Next, we examine whether the prices of the options and the underlying asset co-jump. Figure 
OA.5 shows the frequency of co-jumps events for the short-, medium-, and long-term maturity buckets, respectively. In the vast majority of the cases, an option jump in one delta bucket is not accompanied by simultaneous option jumps in other delta buckets. In line with our findings for the E-mini option market, our results suggest that co-jumps are not common in the S\&P 500 index option market either.

\subsubsection{Information events and information shocks as drivers of option price jumps}

We examine whether the release of scheduled macroeconomic news announcements explains the occurrence of jumps by considering the occurrence of jumps within \pm 10 minutes from the news announcement release. We consider the same news items as in the case the E-mini S\&P 500 options: NFP, CCI, CPI, DGO, FOMC, GDP, IJC, LI, NHS, PPI, and RSA. We calculate the conditional probability $P($ News $\mid$ Jump $)$ as total number jumps occurring within 10 mins from a news announcement divided with the total number of jumps. This provides information on the fraction of jumps associated with news announcements. We also calculate $P(J u m p \mid N e w s)$ as total number jumps occurring within 10 minutes from a news announcement divided with the total number of news announcements. This provides information on whether a news announcement triggers a jump. We consider both the effect of news announcements on aggregate, as well as the effect of each individual news item, separately.

Table OA.2 reports the conditional probabilities $P($ News $\mid J u m p)$ and $P($ Jump $\mid$ News $)$ when we consider all news announcement items jointly (Panel A), as well as each news announcement item separately [Panel B for $P(N e w s \mid J u m p)$ and Panel C for $P(J u m p \mid N e w s)]$. In Table OA.2 Panel A, $6.74 \%$ to $27.59 \%$ of the detected jumps are associated with scheduled macroeconomic news announcements, the majority of which correspond to ATM put options. In the case of the underlying asset (shortest E-mini futures contract), the number of news-related jumps differs from the options ones. This is similar to what we document for the underlying asset of E-mini options where the underlying E-mini futures have the same or nearest maturity to the E-mini options. In addition, $4.72 \%$ to $10.8 \%$ of the news announcements trigger a jump across delta and maturity buckets, which is slightly higher than the $P(J u m p \mid N e w s)$ reported in the main paper for the E-mini options market.

In Panel B, most of the detected option price jumps are associated with IJC news announce- 
ments across all delta and maturity buckets, with $P(N e w s \mid J u m p)$ ranging between $1.44 \%$ to $10.07 \%$. In the case of the underlying, the jumps are associated predominantly with FOMC announcements. In Panel C, NFP announcements followed by CPI announcements trigger most of the option price jumps across delta and maturity buckets, whereas FOMC announcements trigger most jumps in the underlying. The finding that various news items are related to detected jumps in option and futures prices is in tandem with what we document for the E-mini option market.

We now extend our analysis by considering the content of the releases as a determinant of option jumps; market efficiency theory suggest that only news surprises should affect market prices. We investigate whether news surprises affect the conditional probability of a jump occurring and we estimate the following logistic regression model:

$$
P\left(\text { Jump }_{t} \mid N \text { ews }\right)=\frac{1}{1+\exp \left(-c-\sum_{j=1}^{11} \theta_{j}\left|S U R_{j, t}\right|\right)},
$$

where $S U R_{j, t}$ is the surprise of the $j$-th news item $(j=1,2, \ldots, 11$ for NFP, CCI, CPI, DGO, FOMC, GDP, IJC, LI, NHS, PPI, and RSA respectively) and $P\left(J u m p_{t} \mid N e w s\right)$ is the probability of an option price jump to occur conditional on a scheduled macroeconomic announcement taking place ( 1 when there is a jump at the announcement time $t$, and 0 otherwise). We estimate equation (OA.1) conditional on news items being released. Moreover, due to sample size considerations, we estimate equation (OA.1) by pooling all option return observations across delta buckets for any given maturity category. We can see in Table OA.3 Panel A that the content of various news items affects the probability of a jump occurring. For instance, FOMC releases increase the probability of jumps occurring.

Overall, we document that a fraction of the detected option price jumps is associated with scheduled macroeconomic news announcements. In line with our findings for the E-mini S\&P 500 options market, our results further suggest that the segmentation of the option market and the underlying asset holds also around scheduled macroeconomic news announcements, in the sense that the number of news-related jumps in the underlying asset differs substantially from the number of news-related jumps in the option market. 


\subsubsection{Illiquidity and and volume as drivers of option price jumps}

To investigate the role of illiquidity in the occurrence of a jump in S\&P 500 index option market, we augment the logit model given in (OA.1) with three illiquidity variables, namely bid size, ask size, and the standardized bid-ask spread. For a given time stamp, the standardized bid-ask spread is the bid-ask spread divided with standard deviation of the bid-ask spread of the respective delta and maturity category. Table OA.3 Panel B shows that the content of news announcements is significant in a number of cases. Only the ask size is significant across all three maturities. The coefficients of the illiquidity variables are also fairly small (almost zero), suggesting that illiquidity is not a key determinant of jumps in the S\&P 500 index options market.

We verify that illiquidity is not a predominant driver of jumps in the S\&P 500 index option market by ploting the median relative bid-ask spread from 10 minutes before up to 60 minutes after the occurrence of a jump for any given delta and maturity bucket. We can see in Figure OA.6 that the relative bid-ask spread is relatively stable around the occurrence of a jump for any given bucket. This results is in contrast to the findings for the E-mini options market where we document that illiquidity is an important driver of jumps.

We further investigate whether asymmetric information in the spirit described in the main body of the paper, is at play, by augmenting the logit model given in equation (OA.1) with three illiquidity variables, as well as the trading volume in the respective delta and maturity category. Table OA.3 Panel C shows that trading volume is not related with the probability of a jump occurring.

Overall, the results suggest information events and their content are the main drivers of jumps in the S\&P 500 index option prices. This is not the case for the E-mini S\&P 500 option market where illiquidity and asymmetric information among traders plays a key role. 


\section{References}

Andersen, T. G., Bollerslev, T., Diebold, F. X. and Laby, P. (2000). Great realisations, Risk March 2000: 105-108.

Barndorff-Nielsen, O. E., Hansen, P. R., Lunde, A. and Shephard, N. (2009). Realized kernels in practice: Trades and quotes, Econometrics Journal 12: C1-C32.

Black, F. (1976). The pricing of commodity contracts, Journal of Financial Economics 3: 167-179.

Bloomberg Tradebook (2015). In response to overseas demand, CBOE extends VIX, SPX \& SPXW options trading hours.

URL: https://www.bloomberg.com/professional/blog/response-overseas-demand-cboe-extendsvix-spx-options-trading-hours/ [Accessed: 18 July 2019]

Lee, S. S. and Mykland, P. A. (2008). Jumps in financial markets: A new nonparametric test and jump dynamics, Review of Financial Studies 21: 2535-2563.

Stoev, G. (2017). Product focus: What you need to know about S\&P options - \#SaxoStrats.

URL: $\quad$ https://www.tradingfloor.com/posts/product-focus-what-you-need-to-know-about-spoptions-saxostrats-8753750 [Accessed: 18 July 2019] 
Table OA.1: Summary statistics of detected jumps - S\&P 500 index options

\begin{tabular}{ccccccc}
\hline $\begin{array}{c}\text { DOTM } \\
\text { puts }\end{array}$ & $\begin{array}{c}\text { OTM } \\
\text { puts }\end{array}$ & $\begin{array}{c}\text { ATM } \\
\text { puts }\end{array}$ & $\begin{array}{c}\text { ATM } \\
\text { calls }\end{array}$ & $\begin{array}{c}\text { OTM } \\
\text { calls }\end{array}$ & $\begin{array}{c}\text { DOTM } \\
\text { calls }\end{array}$ & Underlying \\
\hline
\end{tabular}

\section{Short-term options}

\# Observations
\# Days
\# Jumps
\# Jump days
P(Jump day)
P(Jump)
Avg. positive jump size
Avg. negative jump size
$\%$ Negative jumps

$\begin{array}{ccccccc}54793 & 54793 & 54793 & 54793 & 54793 & 54793 & 50319 \\ 1479 & 1479 & 1479 & 1479 & 1479 & 1479 & 1436 \\ 671 & 642 & 479 & 545 & 652 & 623 & 54 \\ 310 & 299 & 271 & 263 & 280 & 263 & 43 \\ 20.96 \% & 20.22 \% & 18.32 \% & 17.78 \% & 18.93 \% & 17.78 \% & 2.99 \% \\ 1.22 \% & 1.17 \% & 0.87 \% & 0.99 \% & 1.19 \% & 1.14 \% & 0.11 \% \\ 152.59 \% & 64.00 \% & 43.16 \% & 47.03 \% & 110.33 \% & 182.20 \% & 0.91 \% \\ -67.20 \% & -121.10 \% & -45.27 \% & -78.62 \% & -141.54 \% & -67.71 \% & -0.15 \% \\ 25.34 \% & 59.97 \% & 57.62 \% & 68.62 \% & 51.07 \% & 25.68 \% & 57.41 \%\end{array}$

\section{Medium-term options}

$\begin{array}{lccccccc}\text { \# Observations } & 54817 & 54817 & 54817 & 54818 & 54817 & 54817 & 50319 \\ \text { \# Days } & 1481 & 1481 & 1481 & 1481 & 1481 & 1481 & 1436 \\ \text { \# Jumps } & 306 & 284 & 298 & 300 & 242 & 324 & 54 \\ \text { \# Jump days } & 186 & 177 & 200 & 186 & 144 & 182 & 43 \\ \text { P(Jump day) } & 12.56 \% & 11.95 \% & 13.50 \% & 12.55 \% & 9.72 \% & 12.29 \% & 2.99 \% \\ \text { P(Jump) } & 0.56 \% & 0.52 \% & 0.54 \% & 0.55 \% & 0.44 \% & 0.59 \% & 0.11 \% \\ \text { Avg. positive jump size } & 23.26 \% & 20.82 \% & 17.30 \% & 17.88 \% & 21.03 \% & 29.70 \% & 0.91 \% \\ \text { Avg. negative jump size } & -22.53 \% & -15.37 \% & -14.18 \% & -13.26 \% & -17.02 \% & -43.26 \% & -0.15 \% \\ \text { \% Negative jumps } & 49.02 \% & 47.18 \% & 49.33 \% & 45.33 \% & 39.67 \% & 45.06 \% & 57.41 \%\end{array}$

\section{Long-term options}

$\begin{array}{lccccccc}\text { \# Observations } & 54893 & 54891 & 54891 & 54891 & 54892 & 54897 & 50319 \\ \text { \# Days } & 1492 & 1490 & 1490 & 1490 & 1490 & 1493 & 1436 \\ \text { \# Jumps } & 353 & 352 & 348 & 317 & 321 & 425 & 54 \\ \text { \# Jump days } & 186 & 207 & 219 & 184 & 163 & 198 & 43 \\ \text { P(Jump day) } & 12.47 \% & 13.89 \% & 14.70 \% & 12.35 \% & 10.94 \% & 13.26 \% & 2.99 \% \\ \text { P(Jump) } & 0.64 \% & 0.64 \% & 0.63 \% & 0.58 \% & 0.58 \% & 0.77 \% & 0.11 \% \\ \text { Avg. positive jump size } & 20.53 \% & 16.08 \% & 14.47 \% & 12.75 \% & 14.01 \% & 21.69 \% & 0.91 \% \\ \text { Avg. negative jump size } & -15.13 \% & -11.10 \% & -10.44 \% & -9.19 \% & -13.70 \% & -22.93 \% & -0.15 \% \\ \text { \% Negative jumps } & 49.58 \% & 49.43 \% & 48.56 \% & 46.37 \% & 47.04 \% & 48.24 \% & 57.41 \%\end{array}$

Entries report summary statistics for the detected jumps in 10-minute returns on S\&P 500 cash-settled index options for any given delta and maturity bucket. The number of 10-minute return observations, days, detected jumps, jump days (days with at least one jump), and the probability of a jump day $P($ Jump Day), the probability of a jump occurring $P(J u m p)$, the average size of positive jumps, the average size for negative jumps, and the number of negative jumps as a fraction of all jumps are reported. We use the Lee and Mykland (2008) jump detection methodology based on a significance level of $\alpha=0.1 \%$ to detect jumps. The sample period is January 1, 2005 to December 31, 2010. 
Table OA.2: Relation of jumps and scheduled news announcements - S\&P 500 index options

\begin{tabular}{ccccccc}
\hline DOTM & OTM & ATM & ATM & OTM & DOTM Underlying \\
puts & puts & puts & calls & calls & calls & \\
\hline
\end{tabular}

\section{Panel A: Aggregated over all news items}

\section{Short-term options}

\# Jumps within

10 mins of news

60

60

83

42

61

63

16

P(News $\mid$ Jump)

$8.94 \%$

$9.35 \%$

$17.15 \%$

$6.74 \%$

$9.36 \%$

$11.56 \%$

$21.92 \%$

P(Jump $\mid$ News)

$6.75 \%$

$6.75 \%$

$9.34 \%$

$4.72 \%$

$6.86 \%$

$7.09 \%$

$1.80 \%$

\section{Medium-term options}

\# Jumps within

10 mins of news

$\mathrm{P}$ (News $\mid$ Jump)

58

69

80

50

52

67

16

P(Jump $\mid$ News)

$18.95 \%$

$24.30 \%$

$26.85 \%$

$15.43 \%$

$21.49 \%$

$22.33 \%$

$21.92 \%$

$6.52 \%$

$7.76 \%$

$9.00 \%$

$5.62 \%$

$5.85 \%$

$7.54 \%$

$1.80 \%$

\section{Long-term options}

\# Jumps within

10 mins of news 71

84

96

68

62

85

16

P(News $\mid$ Jump)

$20.11 \%$

$23.86 \%$

$27.59 \%$

$16.00 \%$

$19.31 \% \quad 26.81 \%$

$21.92 \%$

P(Jump|News)

$7.99 \%$

$9.45 \% \quad 10.80 \% \quad 7.65 \%$

$6.97 \% \quad 9.56 \%$

$1.80 \%$

Entries report summary statistics on the relation between detected jumps and scheduled macroeconomic news announcement items for all investigated delta and maturity buckets. The probability of a jump being related to a specific news announcement $P($ News $\mid$ Jump $)$ and the probability of a news announcement leading to a jump $P(J u m p \mid N e w s)$ are reported. Panel A reports these statistics aggregated over all considered news announcement items. Panels B and C report them disaggregated by individual announcement items. Jumps have been detected using the Lee and Mykland (2008) jump detection methodology based on a significance level of $\alpha=0.1 \%$. A jump is defined to be related to news if it occurred within \pm 10 minutes of a scheduled news announcement. The sample period is January 1, 2005 to December 31, 2010. 
Table OA.2: Relation of jumps and scheduled news announcements - S\&P 500 index options

Continued from previous page

\begin{tabular}{ccccccc}
\hline $\begin{array}{c}\text { DOTM } \\
\text { puts }\end{array}$ & $\begin{array}{c}\text { OTM } \\
\text { puts }\end{array}$ & $\begin{array}{c}\text { ATM } \\
\text { puts }\end{array}$ & $\begin{array}{c}\text { ATM } \\
\text { calls }\end{array}$ & $\begin{array}{c}\text { OTM } \\
\text { calls }\end{array}$ & $\begin{array}{c}\text { DOTM } \\
\text { calls }\end{array}$ & Underlying \\
\hline
\end{tabular}

Panel B: P(News / Jump) disaggregated by news items

Short-term options

$\begin{array}{llllllll}\text { NFP } & 1.79 \% & 0.62 \% & 1.45 \% & 1.44 \% & 0.92 \% & 0.73 \% & 0.00 \% \\ \text { CCI } & 0.15 \% & 0.00 \% & 0.41 \% & 0.16 \% & 0.15 \% & 0.18 \% & 0.00 \% \\ \text { CPI } & 0.89 \% & 1.25 \% & 2.07 \% & 0.16 \% & 1.07 \% & 1.65 \% & 0.00 \% \\ \text { DGO } & 1.04 \% & 0.78 \% & 2.07 \% & 0.16 \% & 0.31 \% & 0.73 \% & 1.37 \% \\ \text { FOMC } & 0.60 \% & 0.47 \% & 0.41 \% & 0.16 \% & 0.31 \% & 0.37 \% & 9.59 \% \\ \text { GDP } & 0.45 \% & 1.40 \% & 1.24 \% & 0.00 \% & 0.77 \% & 0.73 \% & 0.00 \% \\ \text { IJC } & 2.53 \% & 3.12 \% & 5.37 \% & 1.44 \% & 2.15 \% & 2.94 \% & 0.00 \% \\ \text { LI } & 0.15 \% & 0.00 \% & 0.00 \% & 0.00 \% & 0.00 \% & 0.00 \% & 0.00 \% \\ \text { NHS } & 0.00 \% & 0.31 \% & 0.00 \% & 0.32 \% & 0.46 \% & 0.55 \% & 0.00 \% \\ \text { PPI } & 0.30 \% & 0.62 \% & 1.86 \% & 0.16 \% & 0.77 \% & 0.92 \% & 0.00 \% \\ \text { RSA } & 0.45 \% & 0.78 \% & 1.45 \% & 0.48 \% & 0.46 \% & 1.10 \% & 0.00 \%\end{array}$

\section{Medium-term options}

$\begin{array}{lccccccc}\text { NFP } & 1.63 \% & 3.17 \% & 3.02 \% & 1.54 \% & 2.48 \% & 2.00 \% & 0.00 \% \\ \text { CCI } & 0.00 \% & 0.00 \% & 0.00 \% & 0.31 \% & 0.00 \% & 0.00 \% & 0.00 \% \\ \text { CPI } & 2.61 \% & 3.17 \% & 3.69 \% & 1.54 \% & 2.48 \% & 3.00 \% & 0.00 \% \\ \text { DGO } & 1.96 \% & 3.52 \% & 3.69 \% & 0.62 \% & 1.65 \% & 2.67 \% & 1.37 \% \\ \text { FOMC } & 0.98 \% & 0.70 \% & 1.01 \% & 0.62 \% & 1.24 \% & 1.33 \% & 9.59 \% \\ \text { GDP } & 1.63 \% & 2.46 \% & 2.68 \% & 0.62 \% & 2.07 \% & 2.33 \% & 0.00 \% \\ \text { IJC } & 6.21 \% & 8.45 \% & 10.07 \% & 5.25 \% & 6.61 \% & 7.00 \% & 0.00 \% \\ \text { LI } & 0.00 \% & 0.00 \% & 0.00 \% & 0.00 \% & 0.00 \% & 0.00 \% & 0.00 \% \\ \text { NHS } & 0.00 \% & 0.00 \% & 0.00 \% & 0.00 \% & 0.00 \% & 0.33 \% & 0.00 \% \\ \text { PPI } & 1.31 \% & 2.11 \% & 3.02 \% & 0.93 \% & 1.65 \% & 2.33 \% & 0.00 \% \\ \text { RSA } & 0.65 \% & 1.06 \% & 1.68 \% & 0.62 \% & 0.83 \% & 1.00 \% & 0.00 \%\end{array}$

\section{Long-term options}

\begin{tabular}{llllllll} 
NFP & $2.27 \%$ & $2.56 \%$ & $4.02 \%$ & $1.65 \%$ & $1.56 \%$ & $2.52 \%$ & $0.00 \%$ \\
CCI & $0.28 \%$ & $0.28 \%$ & $0.00 \%$ & $0.24 \%$ & $0.31 \%$ & $0.32 \%$ & $0.00 \%$ \\
CPI & $1.98 \%$ & $1.99 \%$ & $3.16 \%$ & $0.71 \%$ & $0.93 \%$ & $2.52 \%$ & $0.00 \%$ \\
DGO & $1.13 \%$ & $1.99 \%$ & $2.59 \%$ & $1.18 \%$ & $1.25 \%$ & $1.58 \%$ & $1.37 \%$ \\
FOMC & $0.57 \%$ & $0.57 \%$ & $0.57 \%$ & $0.00 \%$ & $0.00 \%$ & $0.63 \%$ & $9.59 \%$ \\
GDP & $1.70 \%$ & $0.85 \%$ & $1.44 \%$ & $0.71 \%$ & $1.56 \%$ & $2.21 \%$ & $0.00 \%$ \\
IJC & $5.95 \%$ & $5.97 \%$ & $7.18 \%$ & $3.53 \%$ & $5.92 \%$ & $7.26 \%$ & $0.00 \%$ \\
LI & $1.13 \%$ & $0.85 \%$ & $1.15 \%$ & $1.18 \%$ & $1.56 \%$ & $1.26 \%$ & $0.00 \%$ \\
NHS & $0.00 \%$ & $0.28 \%$ & $0.00 \%$ & $0.00 \%$ & $0.31 \%$ & $0.00 \%$ & $0.00 \%$ \\
PPI & $0.57 \%$ & $1.14 \%$ & $0.86 \%$ & $0.24 \%$ & $0.31 \%$ & $0.32 \%$ & $0.00 \%$ \\
RSA & $0.85 \%$ & $1.42 \%$ & $1.72 \%$ & $0.71 \%$ & $1.25 \%$ & $1.26 \%$ & $0.00 \%$ \\
\hline
\end{tabular}


Table OA.2: Relation of jumps and scheduled news announcements - S\&P 500 index options

Continued from previous page

\begin{tabular}{ccccccc}
\hline $\begin{array}{c}\text { DOTM } \\
\text { puts }\end{array}$ & $\begin{array}{c}\text { OTM } \\
\text { puts }\end{array}$ & $\begin{array}{c}\text { ATM } \\
\text { puts }\end{array}$ & $\begin{array}{c}\text { ATM } \\
\text { calls }\end{array}$ & $\begin{array}{c}\text { OTM } \\
\text { calls }\end{array}$ & $\begin{array}{c}\text { DOTM } \\
\text { calls }\end{array}$ & Underlying \\
\end{tabular}

Panel C: P(Jump / News) disaggregated by news items

Short-term options

$\begin{array}{lccccccc}\text { CCI } & 1.39 \% & 0.00 \% & 2.78 \% & 1.39 \% & 1.39 \% & 1.39 \% & 0.00 \% \\ \text { CPI } & 8.45 \% & 11.27 \% & 14.08 \% & 1.41 \% & 9.86 \% & 12.68 \% & 0.00 \% \\ \text { DGO } & 9.72 \% & 6.94 \% & 13.89 \% & 1.39 \% & 2.78 \% & 5.56 \% & 1.39 \% \\ \text { FOMC } & 8.00 \% & 6.00 \% & 4.00 \% & 2.00 \% & 4.00 \% & 4.00 \% & 14.00 \% \\ \text { GDP } & 4.17 \% & 12.50 \% & 8.33 \% & 0.00 \% & 6.94 \% & 5.56 \% & 0.00 \% \\ \text { IJC } & 5.45 \% & 6.41 \% & 8.33 \% & 2.88 \% & 4.49 \% & 5.13 \% & 0.00 \% \\ \text { LI } & 1.39 \% & 0.00 \% & 0.00 \% & 0.00 \% & 0.00 \% & 0.00 \% & 0.00 \% \\ \text { NFP } & 17.14 \% & 5.71 \% & 10.00 \% & 12.86 \% & 8.57 \% & 5.71 \% & 0.00 \% \\ \text { NHS } & 0.00 \% & 2.82 \% & 0.00 \% & 2.82 \% & 4.23 \% & 4.23 \% & 0.00 \% \\ \text { PPI } & 2.78 \% & 5.56 \% & 12.50 \% & 1.39 \% & 6.94 \% & 6.94 \% & 0.00 \% \\ \text { RSA } & 4.29 \% & 7.14 \% & 10.00 \% & 4.29 \% & 4.29 \% & 8.57 \% & 0.00 \%\end{array}$

\section{Medium-term options}

$\begin{array}{lccccccc}\text { CCI } & 0.00 \% & 0.00 \% & 0.00 \% & 1.39 \% & 0.00 \% & 0.00 \% & 0.00 \% \\ \text { CPI } & 11.27 \% & 12.68 \% & 15.49 \% & 7.04 \% & 8.45 \% & 12.68 \% & 0.00 \% \\ \text { DGO } & 8.33 \% & 13.89 \% & 15.28 \% & 2.78 \% & 5.56 \% & 11.11 \% & 1.39 \% \\ \text { FOMC } & 6.00 \% & 4.00 \% & 6.00 \% & 4.00 \% & 6.00 \% & 8.00 \% & 14.00 \% \\ \text { GDP } & 6.94 \% & 9.72 \% & 11.11 \% & 2.78 \% & 6.94 \% & 9.72 \% & 0.00 \% \\ \text { IJC } & 6.09 \% & 7.69 \% & 9.62 \% & 5.45 \% & 5.13 \% & 6.73 \% & 0.00 \% \\ \text { LI } & 0.00 \% & 0.00 \% & 0.00 \% & 0.00 \% & 0.00 \% & 0.00 \% & 0.00 \% \\ \text { NFP } & 7.14 \% & 12.86 \% & 12.86 \% & 7.14 \% & 8.57 \% & 8.57 \% & 0.00 \% \\ \text { NHS } & 0.00 \% & 0.00 \% & 0.00 \% & 0.00 \% & 0.00 \% & 1.41 \% & 0.00 \% \\ \text { PPI } & 5.56 \% & 8.33 \% & 12.50 \% & 4.17 \% & 5.56 \% & 9.72 \% & 0.00 \% \\ \text { RSA } & 2.86 \% & 4.29 \% & 7.14 \% & 2.86 \% & 2.86 \% & 4.29 \% & 0.00 \%\end{array}$

\section{Long-term options}

\begin{tabular}{lccccccc} 
CCI & $1.39 \%$ & $1.39 \%$ & $0.00 \%$ & $1.39 \%$ & $1.39 \%$ & $1.39 \%$ & $0.00 \%$ \\
CPI & $9.86 \%$ & $9.86 \%$ & $15.49 \%$ & $4.23 \%$ & $4.23 \%$ & $11.27 \%$ & $0.00 \%$ \\
DGO & $5.56 \%$ & $9.72 \%$ & $12.50 \%$ & $6.94 \%$ & $5.56 \%$ & $6.94 \%$ & $1.39 \%$ \\
FOMC & $4.00 \%$ & $4.00 \%$ & $4.00 \%$ & $0.00 \%$ & $0.00 \%$ & $4.00 \%$ & $14.00 \%$ \\
GDP & $8.33 \%$ & $4.17 \%$ & $6.94 \%$ & $4.17 \%$ & $6.94 \%$ & $9.72 \%$ & $0.00 \%$ \\
IJC & $6.73 \%$ & $6.73 \%$ & $8.01 \%$ & $4.81 \%$ & $6.09 \%$ & $7.37 \%$ & $0.00 \%$ \\
LI & $5.56 \%$ & $4.17 \%$ & $5.56 \%$ & $6.94 \%$ & $6.94 \%$ & $5.56 \%$ & $0.00 \%$ \\
NFP & $11.43 \%$ & $12.86 \%$ & $20.00 \%$ & $10.00 \%$ & $7.14 \%$ & $11.43 \%$ & $0.00 \%$ \\
NHS & $0.00 \%$ & $1.41 \%$ & $0.00 \%$ & $0.00 \%$ & $1.41 \%$ & $0.00 \%$ & $0.00 \%$ \\
PPI & $2.78 \%$ & $5.56 \%$ & $4.17 \%$ & $1.39 \%$ & $1.39 \%$ & $1.39 \%$ & $0.00 \%$ \\
RSA & $4.29 \%$ & $7.14 \%$ & $8.57 \%$ & $4.29 \%$ & $5.71 \%$ & $5.71 \%$ & $0.00 \%$ \\
\hline
\end{tabular}


Table OA.3: Information shocks, volume, and illiquidity as jump determinants - S\&P 500 index options

\begin{tabular}{lccc}
\hline & Short-term & Medium-term & Long-term \\
\hline & & & \\
Panel A: News covariates & & \\
$\mathrm{c}$ & -2.658 & $-2.607^{* * *}$ & $-2.539^{* * *}$ \\
$C C I_{t}$ & $-1.217^{* * *}$ & -601.630 & $-4.987^{* * *}$ \\
$C P I_{t}$ & 0.036 & $0.327^{*}$ & -0.199 \\
$D G O_{t}$ & 0.186 & $0.442^{* * *}$ & $0.282^{*}$ \\
$F O M C_{t}$ & $0.465^{* * *}$ & $0.522^{* * *}$ & $0.274^{*}$ \\
$G D P_{t}$ & -0.021 & 0.124 & -0.120 \\
$I J C_{t}$ & -0.013 & 0.026 & -0.041 \\
$L I_{t}$ & $-3.478^{* * *}$ & -39.272 & -0.156 \\
$N F P_{t}$ & $0.350^{* *}$ & $0.563^{* * *}$ & $0.721^{* * *}$ \\
$N H S_{t}$ & $-0.747^{* *}$ & $-4.588^{* * *}$ & $-4.810^{* * *}$ \\
$P P I_{t}$ & $-0.563^{*}$ & 0.157 & $-2.237^{* * *}$ \\
$R S A_{t}$ & -0.209 & $-1.389^{* * *}$ & -0.287
\end{tabular}

Panel B: News and liquidity covariates

$\begin{array}{lccc}\text { c } & -2.907^{* * *} & -2.944^{* * *} & -2.895^{* * *} \\ \text { BidSize }_{t-1} & 0.000 & 0.001 & 0.001 \\ \text { AskSize }_{t-1} & 0.002^{* * *} & 0.002^{* *} & 0.002^{* *} \\ s B A_{t-1} & 0.000 & 0.000 & 0.000^{* * *} \\ C C I_{t} & -1.171^{* *} & -5.128^{*} & -5.611^{* * *} \\ C P I_{t} & 0.062 & 0.383^{* *} & -0.294 \\ D G O_{t} & 0.060 & 0.384^{* *} & 0.238 \\ F O M C_{t} & 0.407^{* * *} & 0.543^{* * *} & 0.178 \\ G D P_{t} & -0.096 & 0.073 & -0.107 \\ I J C_{t} & -0.011 & 0.058 & 0.016 \\ \text { II }_{t} & -4.431^{* *} & -29.16 & -0.401 \\ N F P_{t} & 0.442^{* * *} & 0.647^{* * *} & 0.688^{* * *} \\ N H S_{t} & -0.571 & -4.500^{* * *} & -4.760^{* * *} \\ \text { PPI }_{t} & -0.570^{*} & 0.222 & -2.348^{* * *} \\ R S A_{t} & -0.127 & -1.081^{* *} & -0.193\end{array}$

Panel C: News, volume, and liquidity covariates

$\begin{array}{lccc}\text { c } & -2.821^{* * *} & -2.850^{* * *} & -2.611^{* * *} \\ \text { BidSize }_{t-1} & 0.000 & 0.000 & 0.000 \\ \text { AskSize }_{t-1} & 0.002^{* *} & 0.002^{*} & 0.001 \\ \text { sBA }_{t-1} & 0.000 & 0.000 & 0.000^{* * *} \\ \text { Volume }_{t-1, t} & 0.000 & 0.000 & 0.000 \\ \text { CCI } & -1.232^{* * *} & -5.329^{* * *} & -5.771^{* * *} \\ \text { CPI } & -0.035 & 0.340^{* *} & -0.076 \\ \text { DGO }_{t} & 0.027 & 0.368^{* *} & 0.171 \\ F O M C_{t} & 0.400^{* * *} & 0.528^{* * *} & 0.160 \\ \text { FDP }_{t} & -0.128 & 0.050 & -0.153 \\ \text { IJC }_{t} & 0.024 & 0.084 & 0.077 \\ \text { LI }_{t} & -4.542^{* *} & -29.295 & -0.323 \\ N F P_{t} & 0.466^{* * *} & 0.616^{* * *} & 0.627^{* * *} \\ N H S_{t} & -0.628^{*} & -4.629^{* * *} & -4.834^{* * *} \\ \text { PPI }_{t} & -0.633^{*} & 0.208 & -2.078^{* * *} \\ \text { RSA }_{t} & -0.150 & -0.878^{* *} & -0.012\end{array}$

Entries report the estimation results for the logistic regression models in equations (OA.1) [Panel A], as well as the logistic model augmented with illiquidity variables [Panel B] and trading volume [Panel C]. The estimation is performed separately for short-, medium-, and long-term options on a sample pooled across all delta categories. Only news-related observations are considered. ${ }^{* * *}$, ${ }^{* *}$, and ${ }^{*}$ report statistical significance at the $1 \%, 5 \%$, and $10 \%$ levels, respectively. The sample period is January 1, 2005 to December 31, 2010. 
Figure OA.1: Volatility signature plots of short-term options returns

Panel A: Short-term options
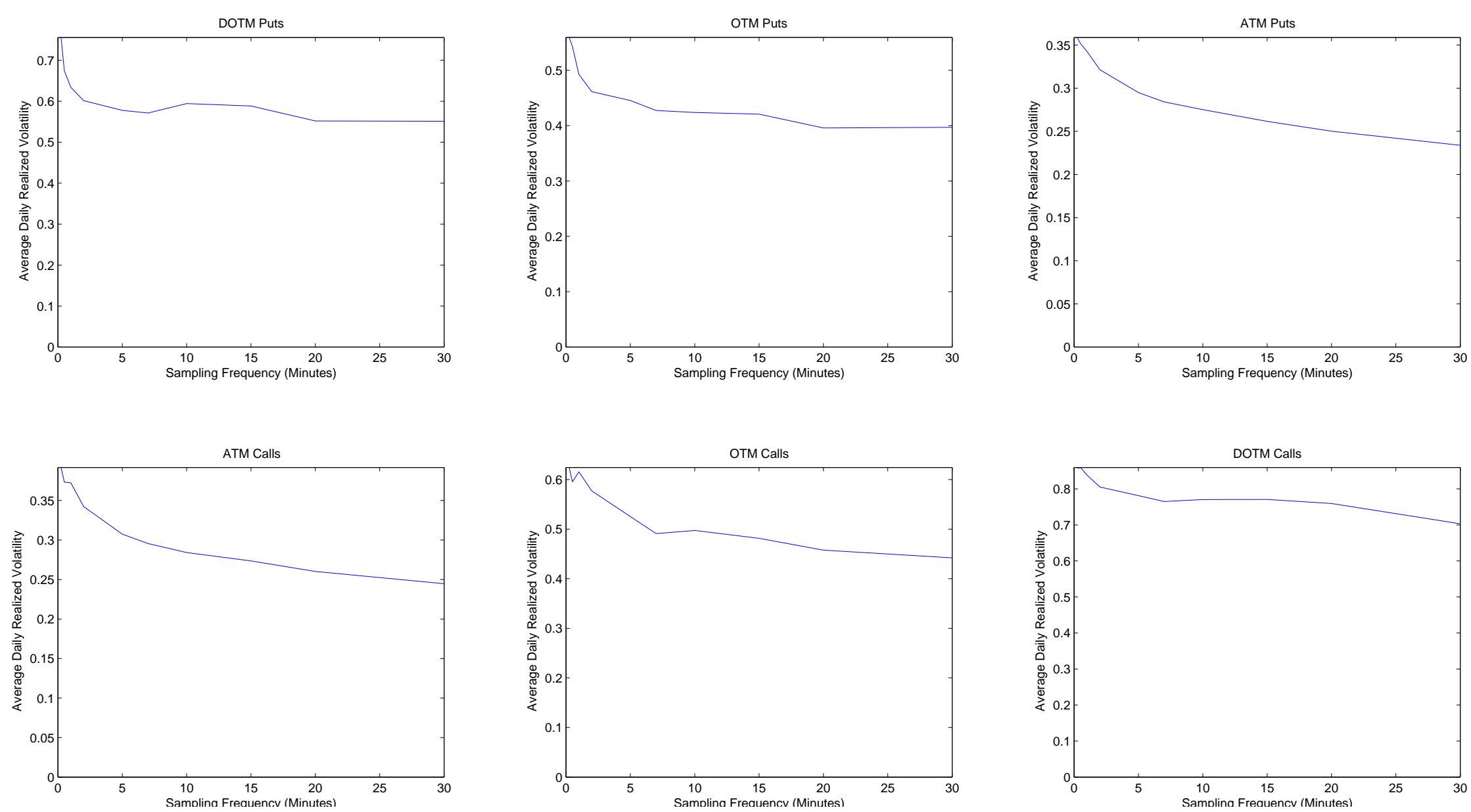
Figure OA.1: Volatility signature plots

Continued from previous page

Panel B: Medium-term options
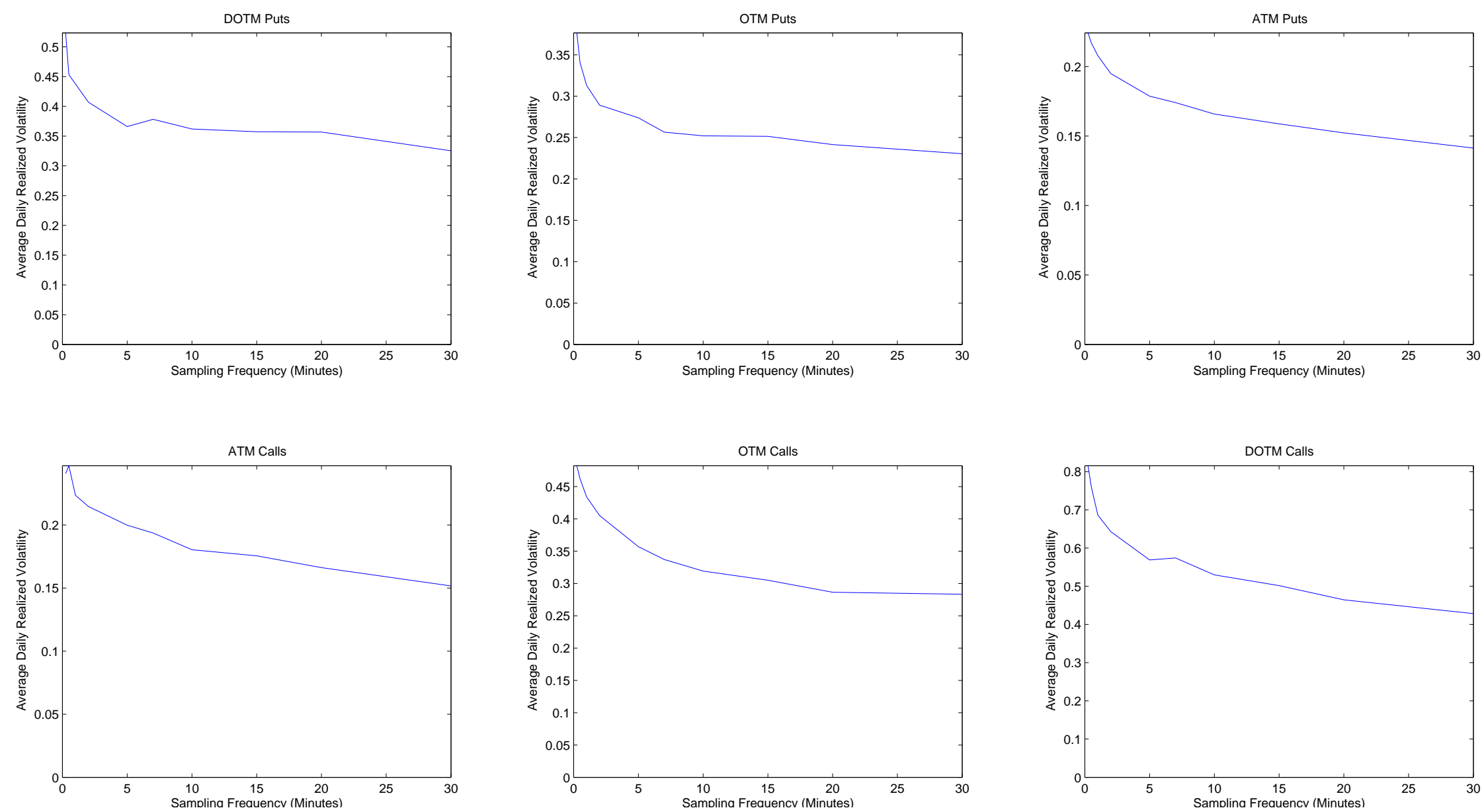


\section{Figure OA.1: Volatility signature plots}

\section{Continued from previous page}

\section{Panel C: Long-term options}
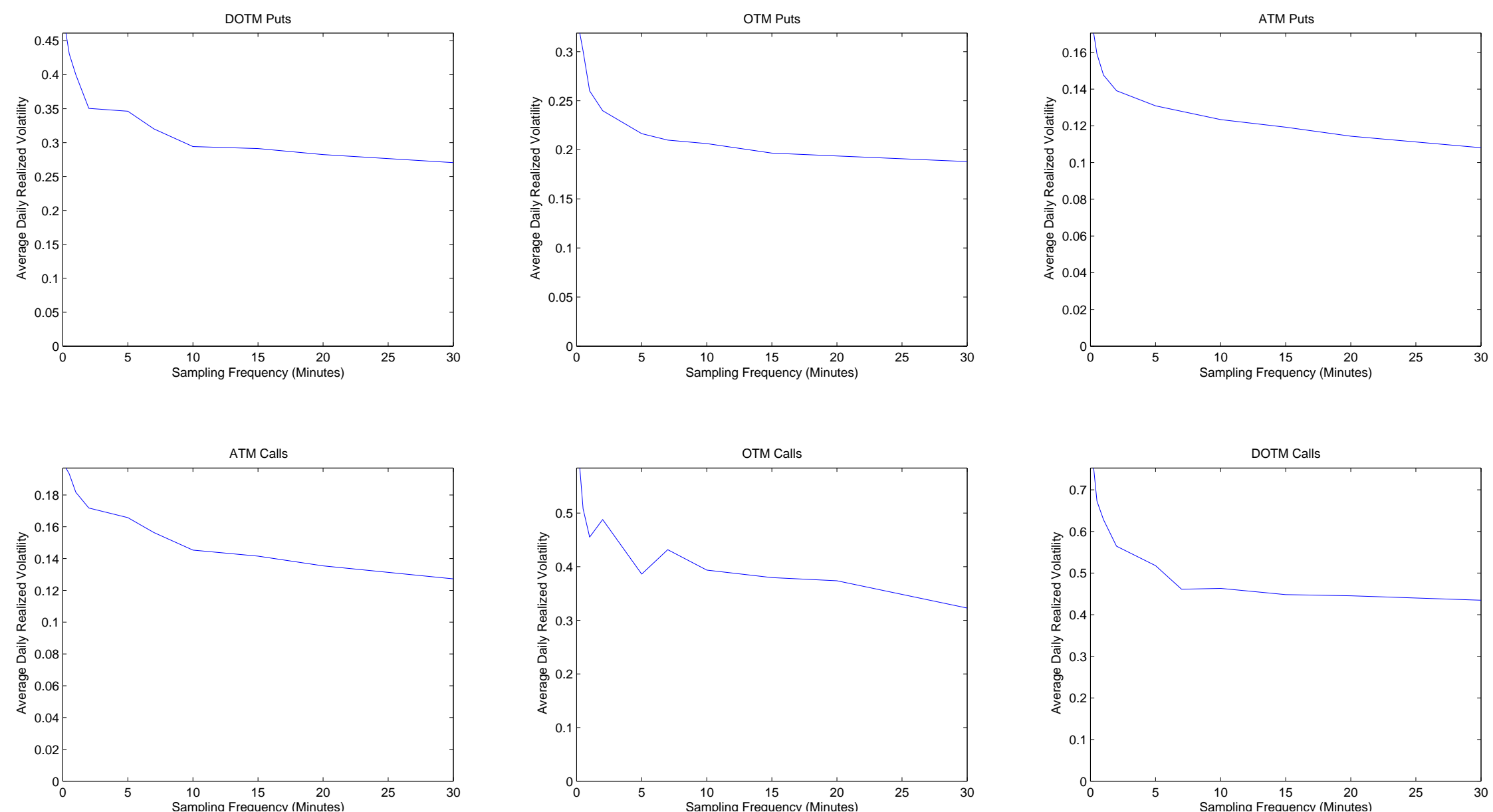

The figure depicts the average daily realized volatility of option returns as a function of the sampling frequency for short-, medium-, and long-term options (Panels A, B, and C, respectively) of different delta categories. We consider the following delta categories for calls and puts (as defined in Table 1 in the main paper): deep out-of-the-money (DOTM), out-of-the-money (OTM), and at-the-money (ATM). 
Figure OA.2: Broadening the definition of concurrent jumps

Panel A: Short-term options
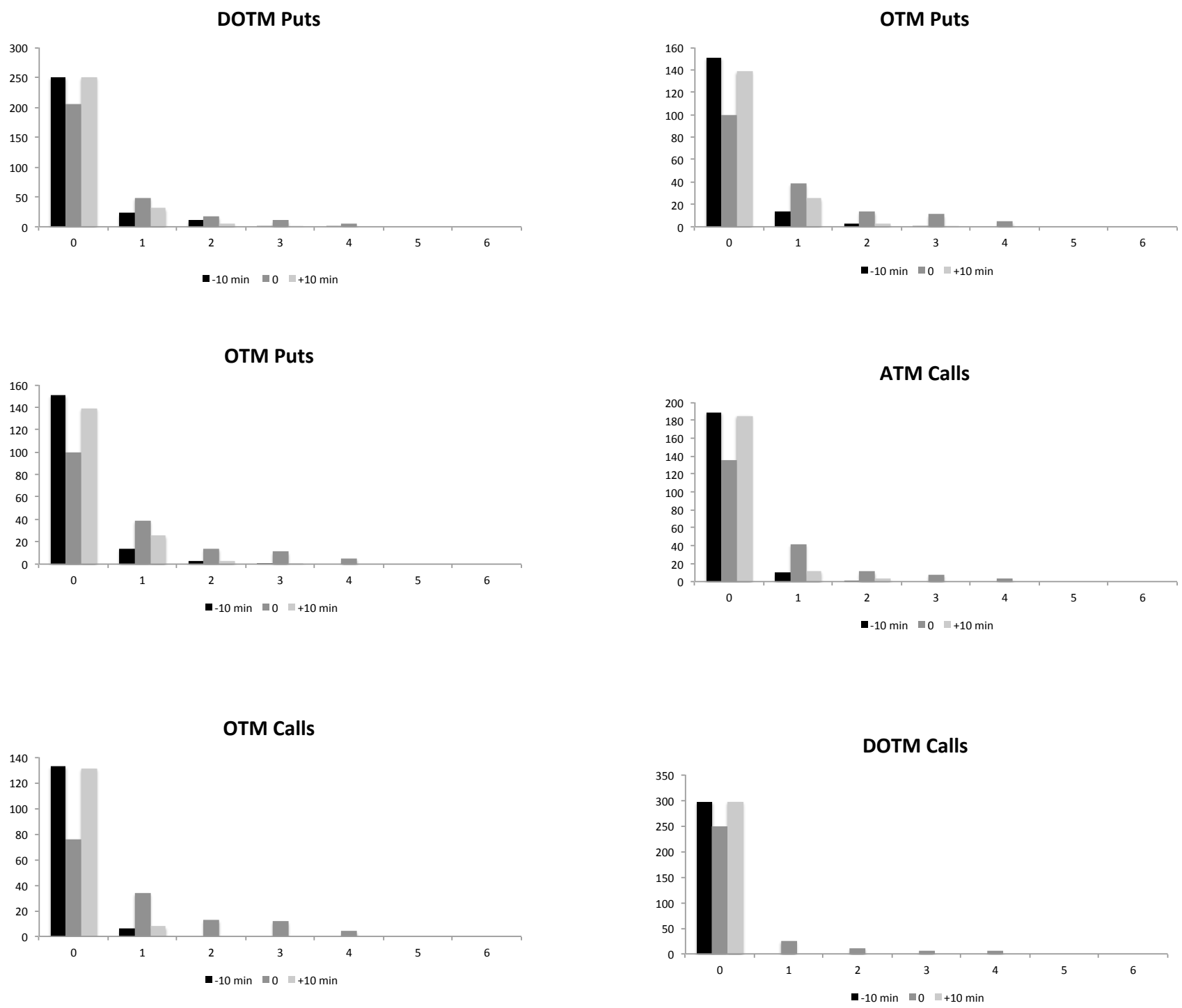
Figure OA.2: Broadening the definition of concurrent jumps Continued from previous page

Panel B: Medium-term options
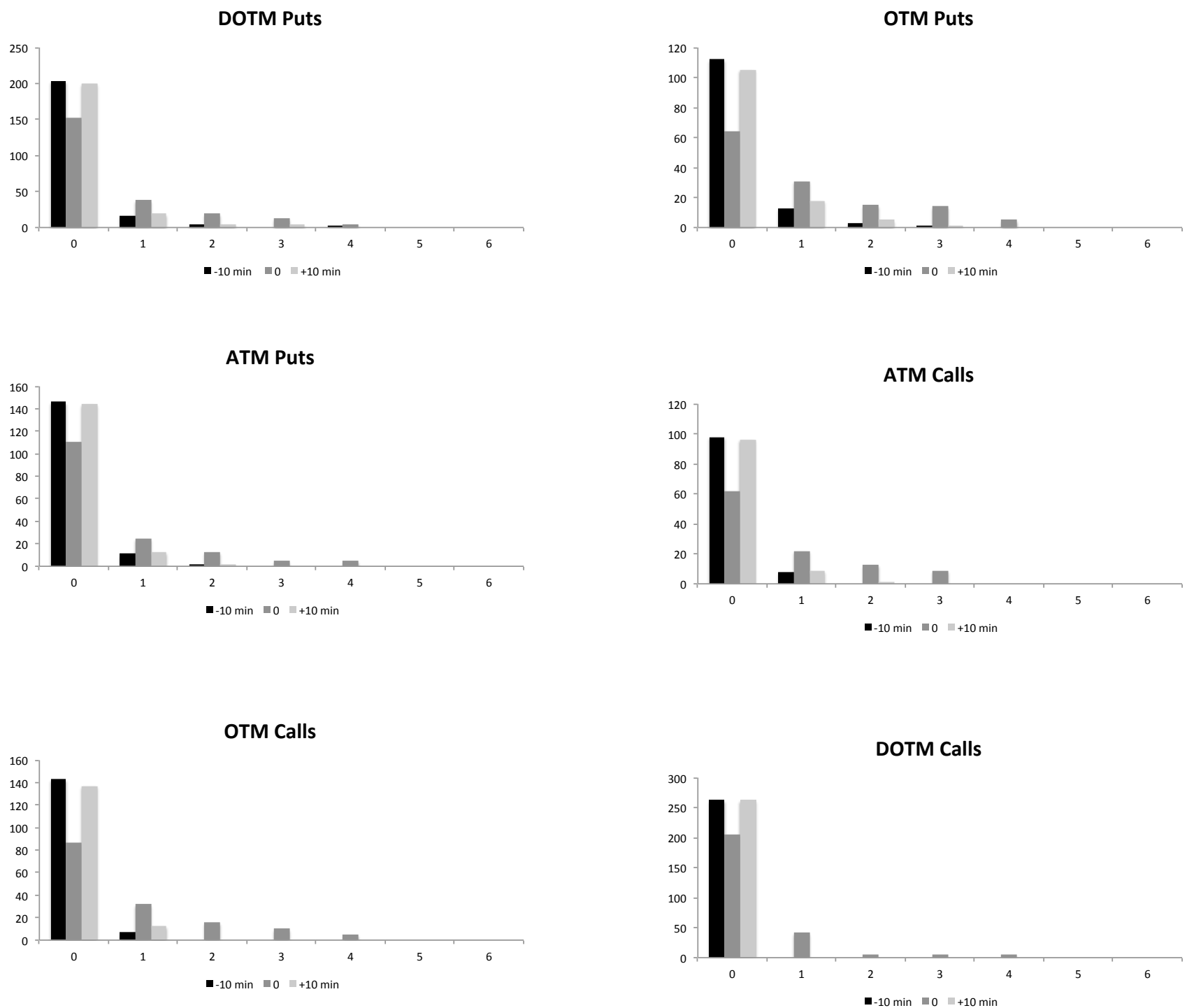
Figure OA.2: Broadening the definition of concurrent jumps

Continued from previous page

\section{Panel C: Long-term options}


ATM Puts
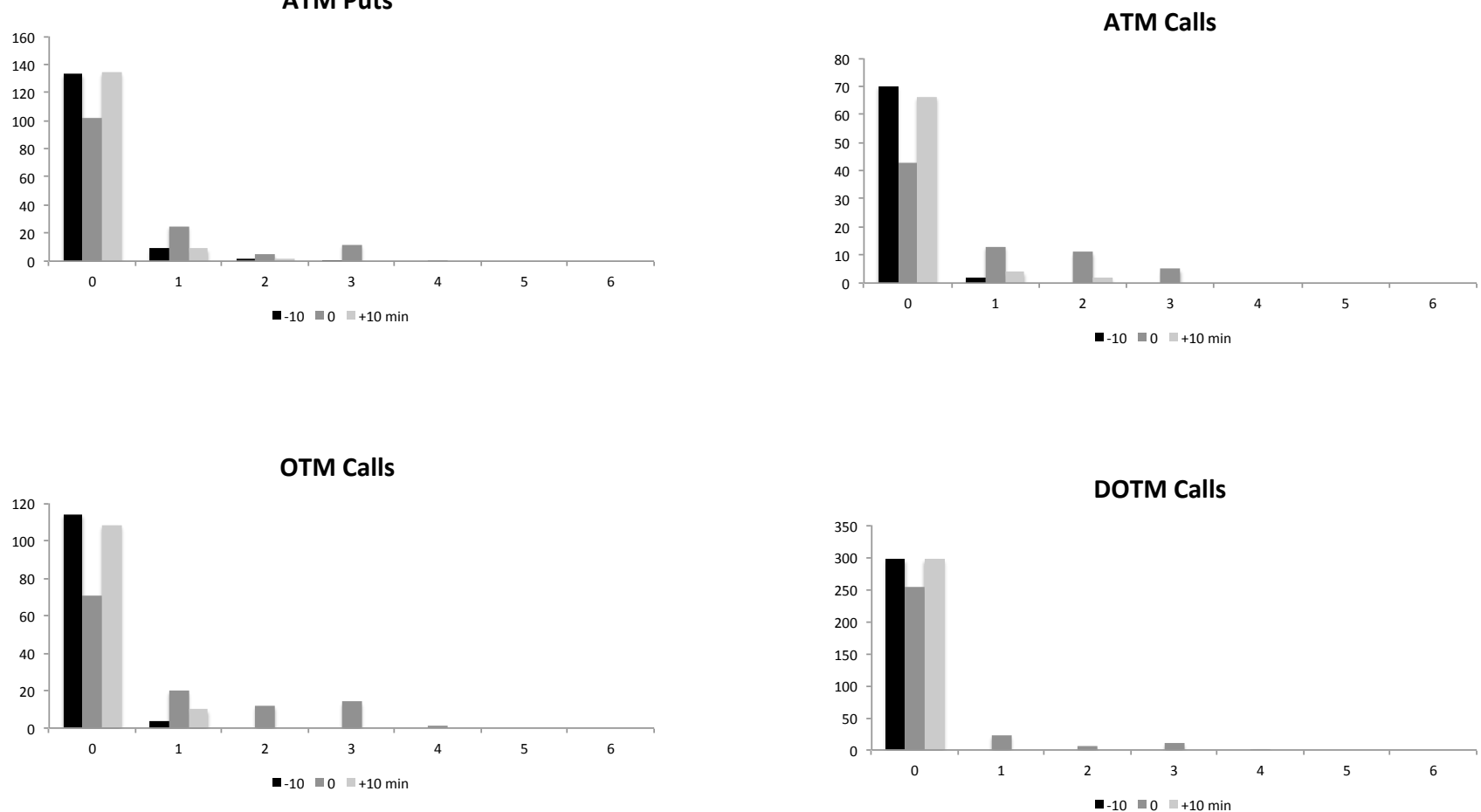

Focusing on each maturity and delta bucket separately the figure shows the number of jumps that the remaining categories exhibit 10 minutes before, simultaneously, and 10 minutes after we detect a jump in the bucket under consideration for short-, medium-, and long-term options (Panels A, B, and C, respectively). For each maturity category, we consider deep out-of-the-money (DOTM), out-of-the-money (OTM), and at-the-money (ATM) calls and puts, as defined in Table 1 in the main paper. 
Figure OA.3: Distribution of option trading volume before news-related option price jumps

Panel A: Short-term options

Figure OA.3: Distribution of option trading volume before news-related option price jumps

Continued from previous page

Panel B: Medium-term options
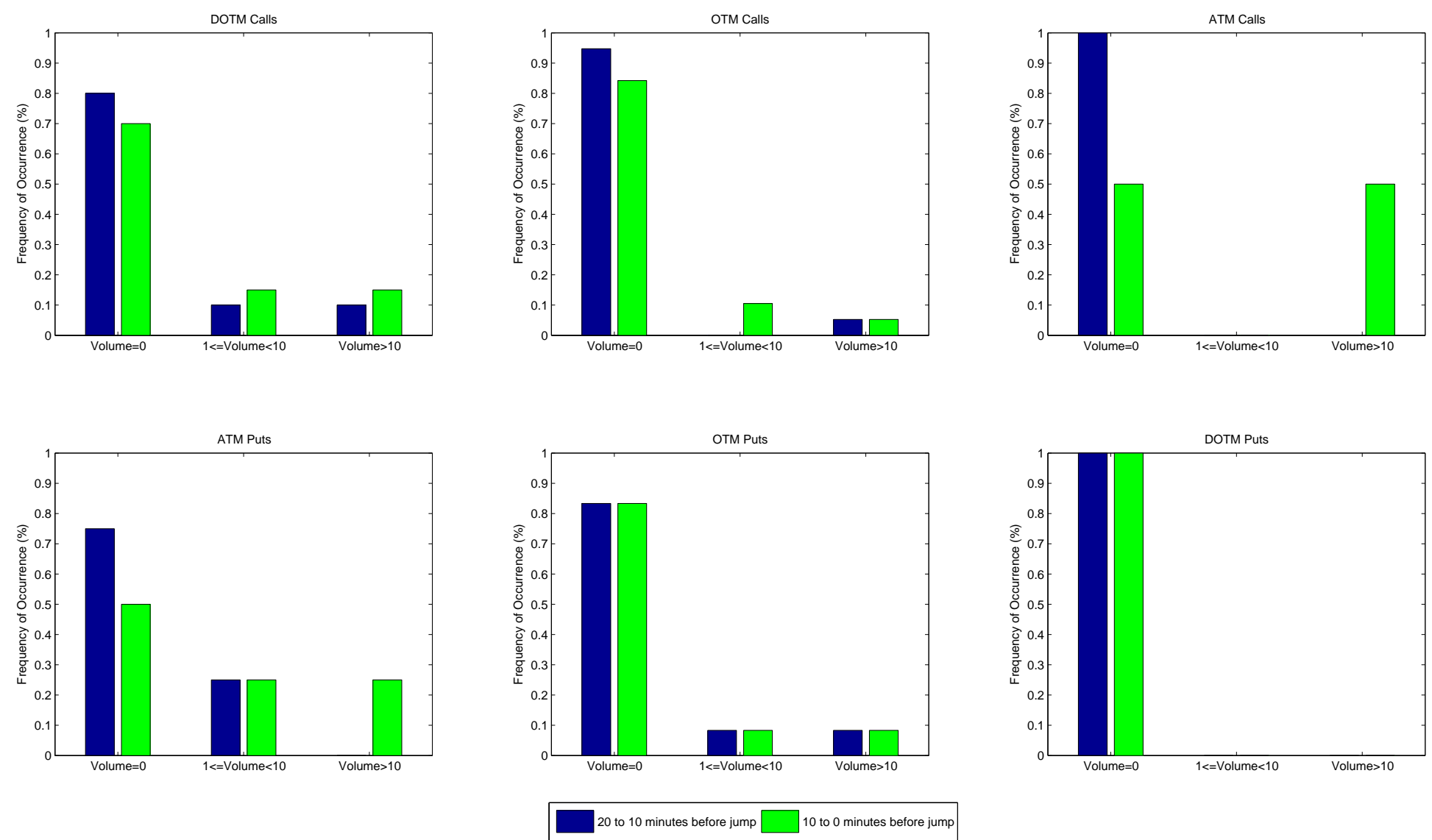
Figure OA.3: Distribution of option trading volume before news-related option price jumps

Continued from previous page

Panel C: Long-term options
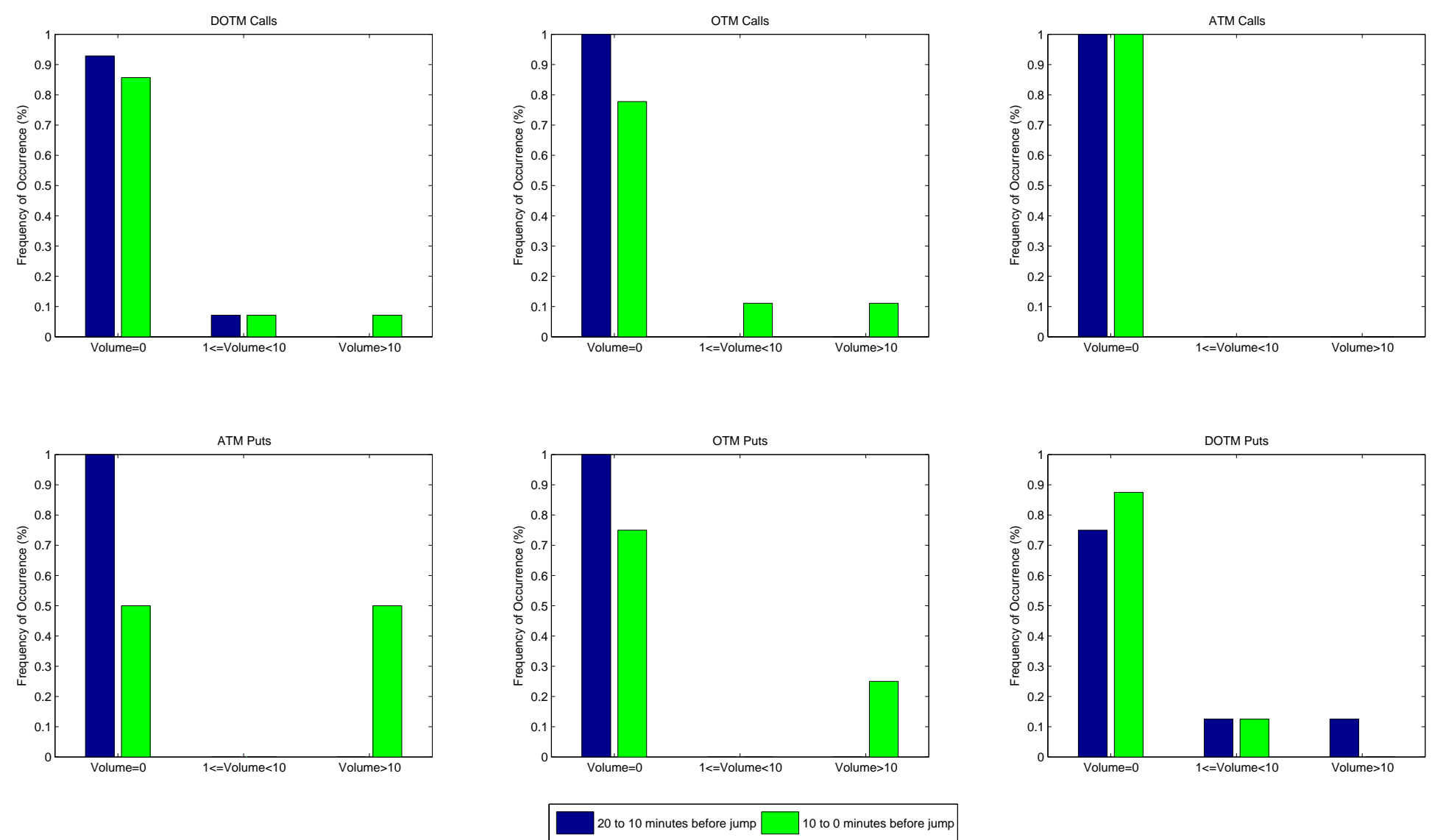

The figure illustrates the frequency (in \%) distribution in the two 10-minute subintervals before news-related short-, medium-, and long-term option price jumps (Panels A, B, and C, respectively). The frequency distribution is constructed for option trading volume buckets of zero, ten or more than ten contracts. For each maturity category, we consider deep out-of-the-money (DOTM), out-of-the-money (OTM), and at-the-money (ATM) calls and puts, as defined in Table 1 in the main paper. 
Figure OA.4: Dynamics of options' bid-ask spreads

Panel A: Short-term options
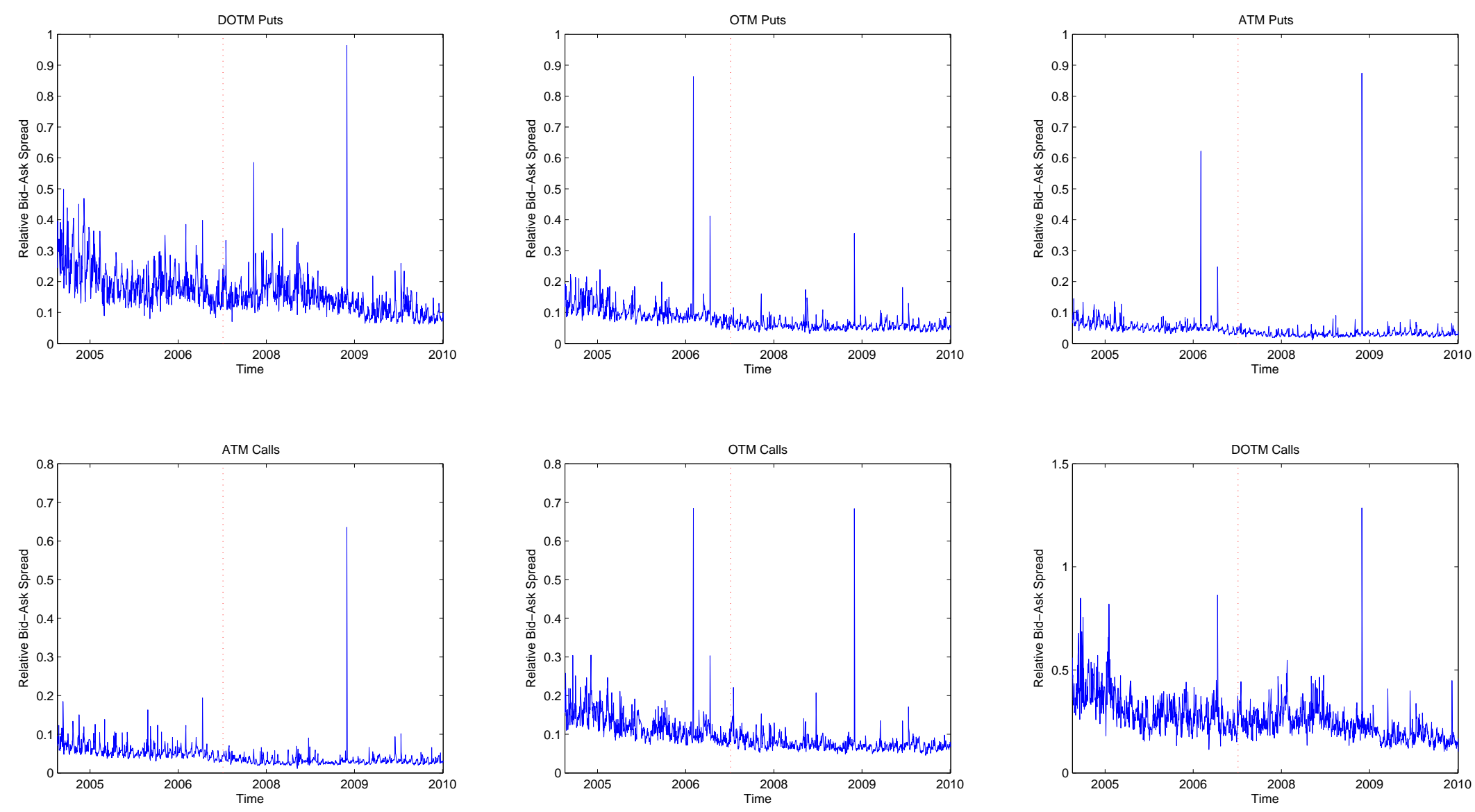
Figure OA.4: Dynamics of options' bid-ask spreads

Continued from previous page

Panel B: Medium-term options
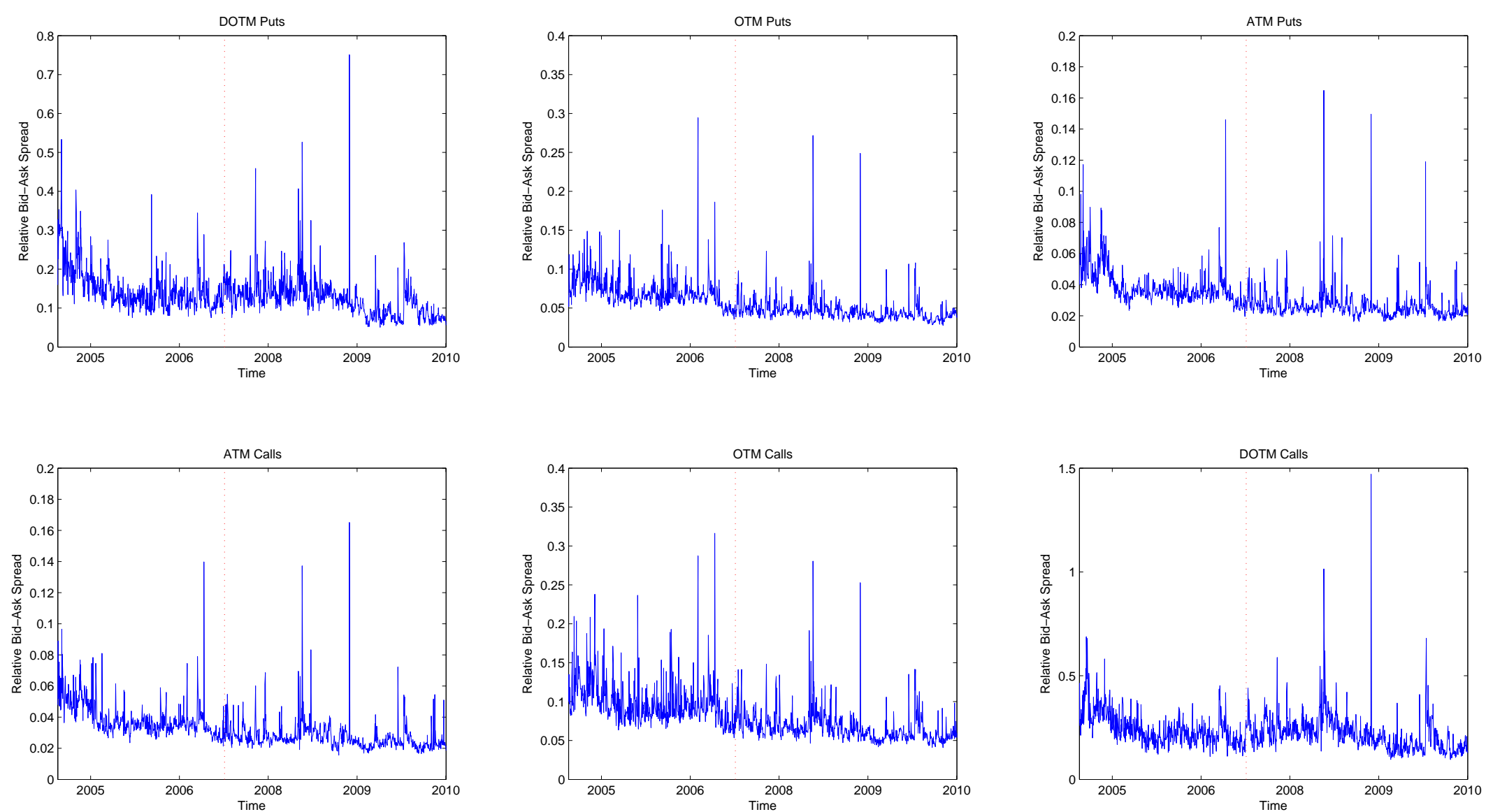
Figure OA.4: Dynamics of options' bid-ask spreads

Continued from previous page

\section{Panel C: Long-term options}
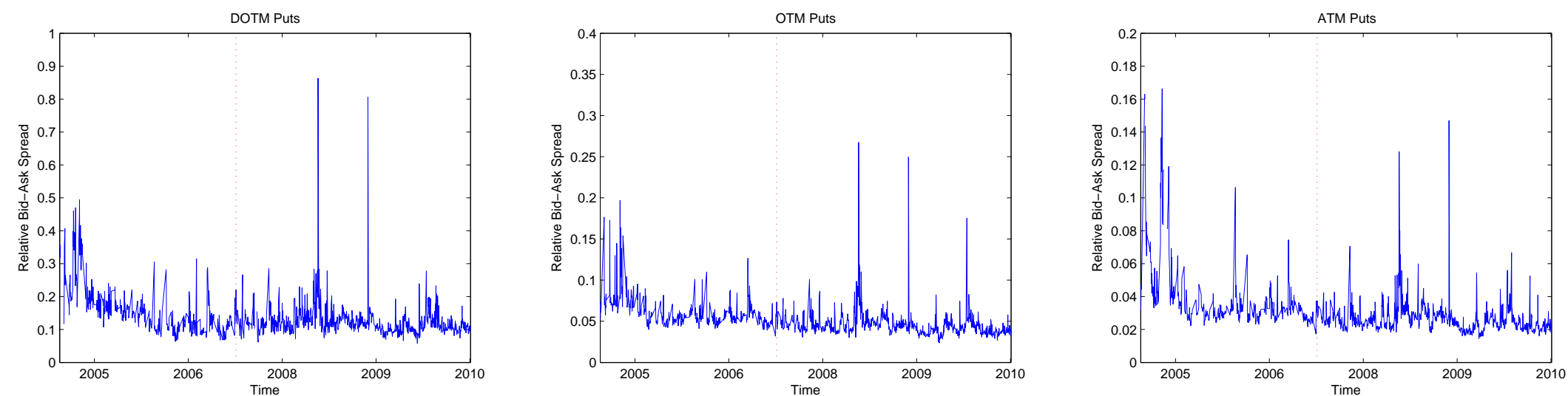

N
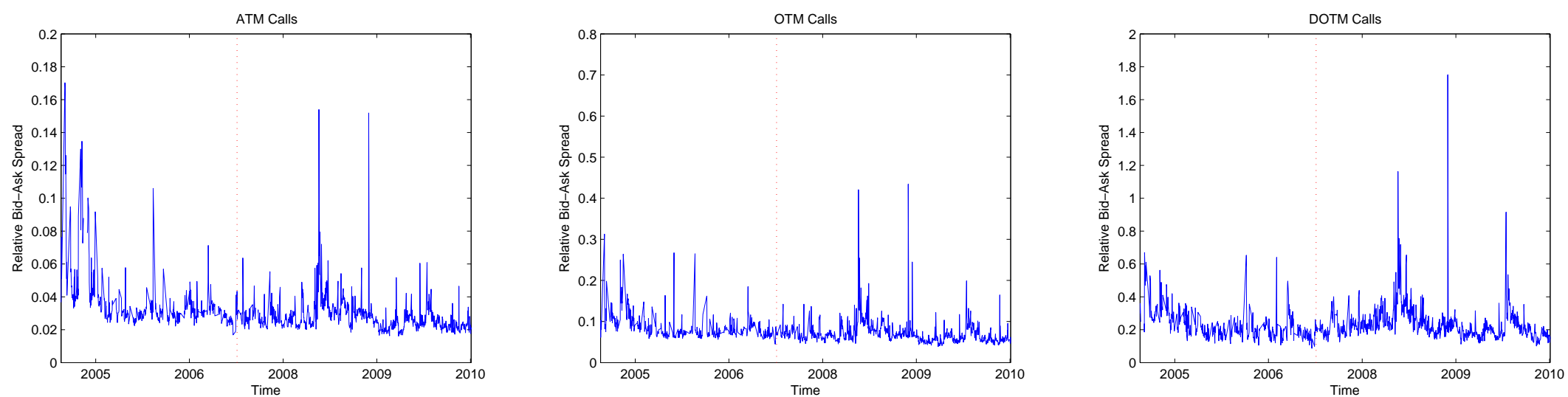

The figure illustrates the time evolution of the relative bid-ask spread of short-, medium-, and long-term options (Panels A, B, and C, respectively) of different delta categories over the non-crisis and crisis subsamples. The daily average relative-bid ask spread is depicted. The dashed line illustrates the non-crisis/crisis split point. For each maturity category, we consider deep out-of-the-money (DOTM), out-of-the-money (OTM), and at-the-money (ATM) calls and puts, as defined in Table 1 in the main paper. 
Figure OA.5: Distribution of co-Jumps - S\&P 500 index options

Panel A: Short-term options

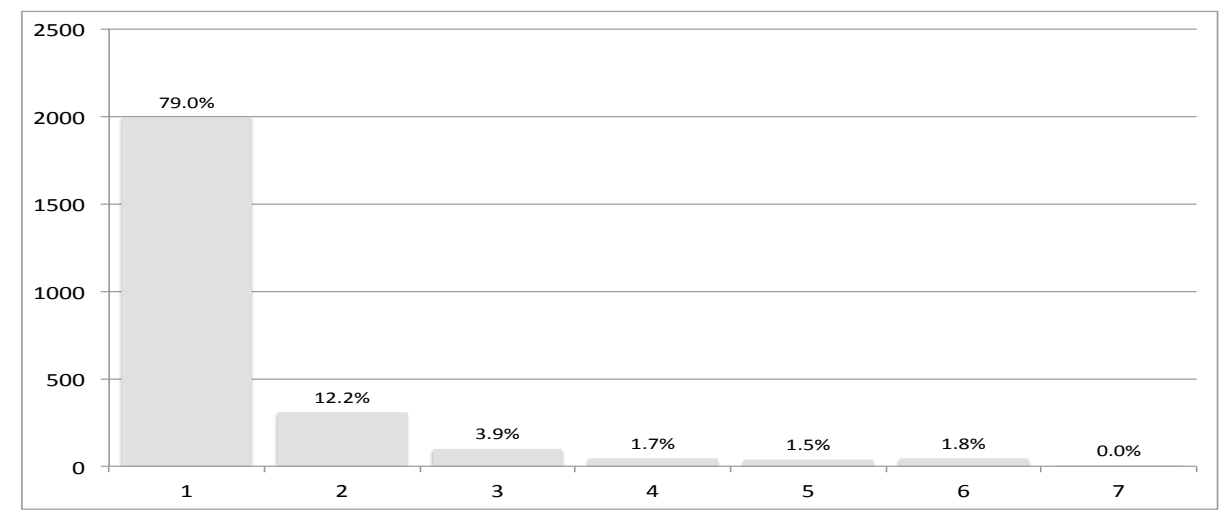

Panel B: Medium-term options

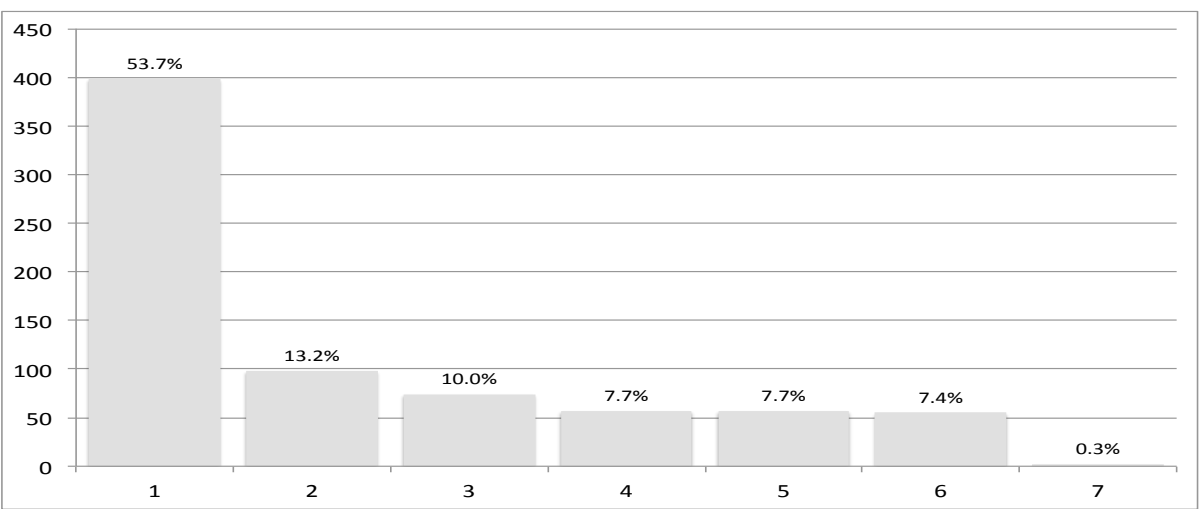

Panel C: Long-term options

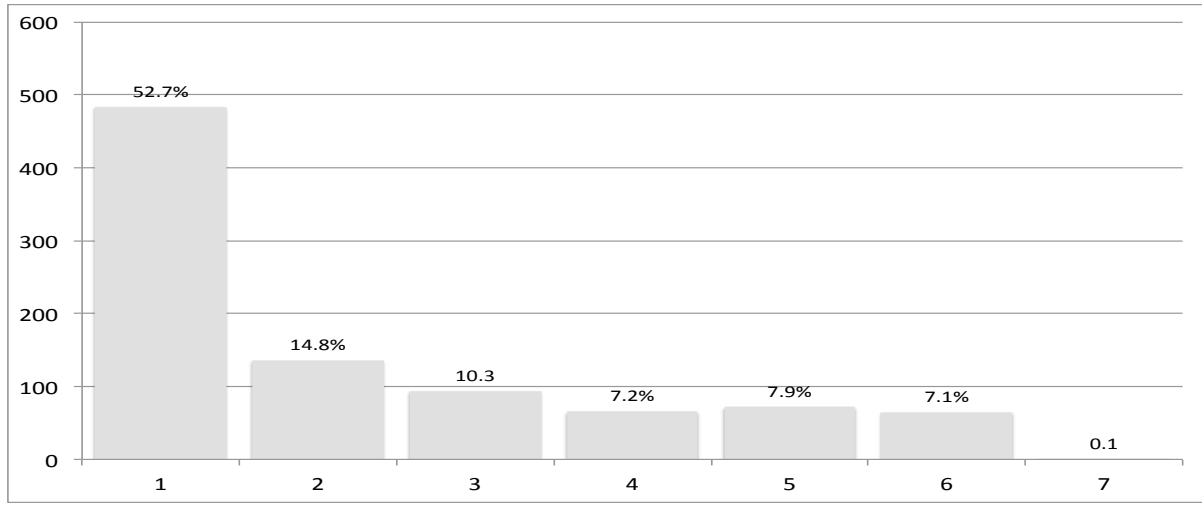

The figure illustrates the distribution of co-jump events for short-, medium-, and long-term options (Panels A, B, and C, respectively) in the case of S\&P 500 options. Co-jump events are defined by the number of concurrent jumps across different delta levels and the underlying asset. The event of only one concurrent jump corresponds to an idiosyncratic jump in only one of the delta categories or the underlying asset. The frequency of occurrence is reported for each possible co-jump event. 
Figure OA.6: Option bid-ask spreads around jumps - S\&P 500 index options

Panel A: Short-term options
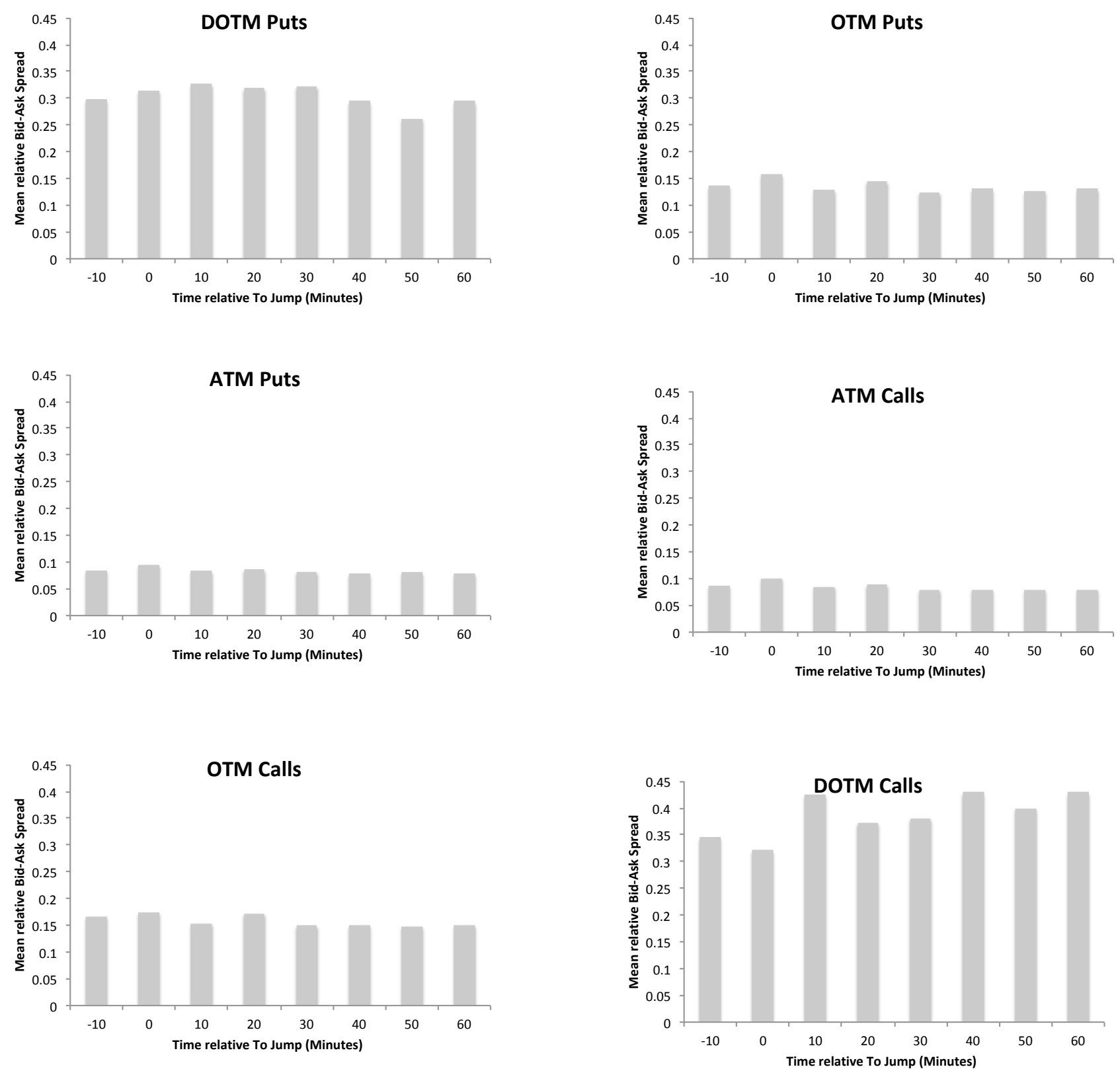
Figure OA.6: Option bid-ask spreads around jumps - S\&P 500 index options Continued from previous page

Panel B: Medium-term options
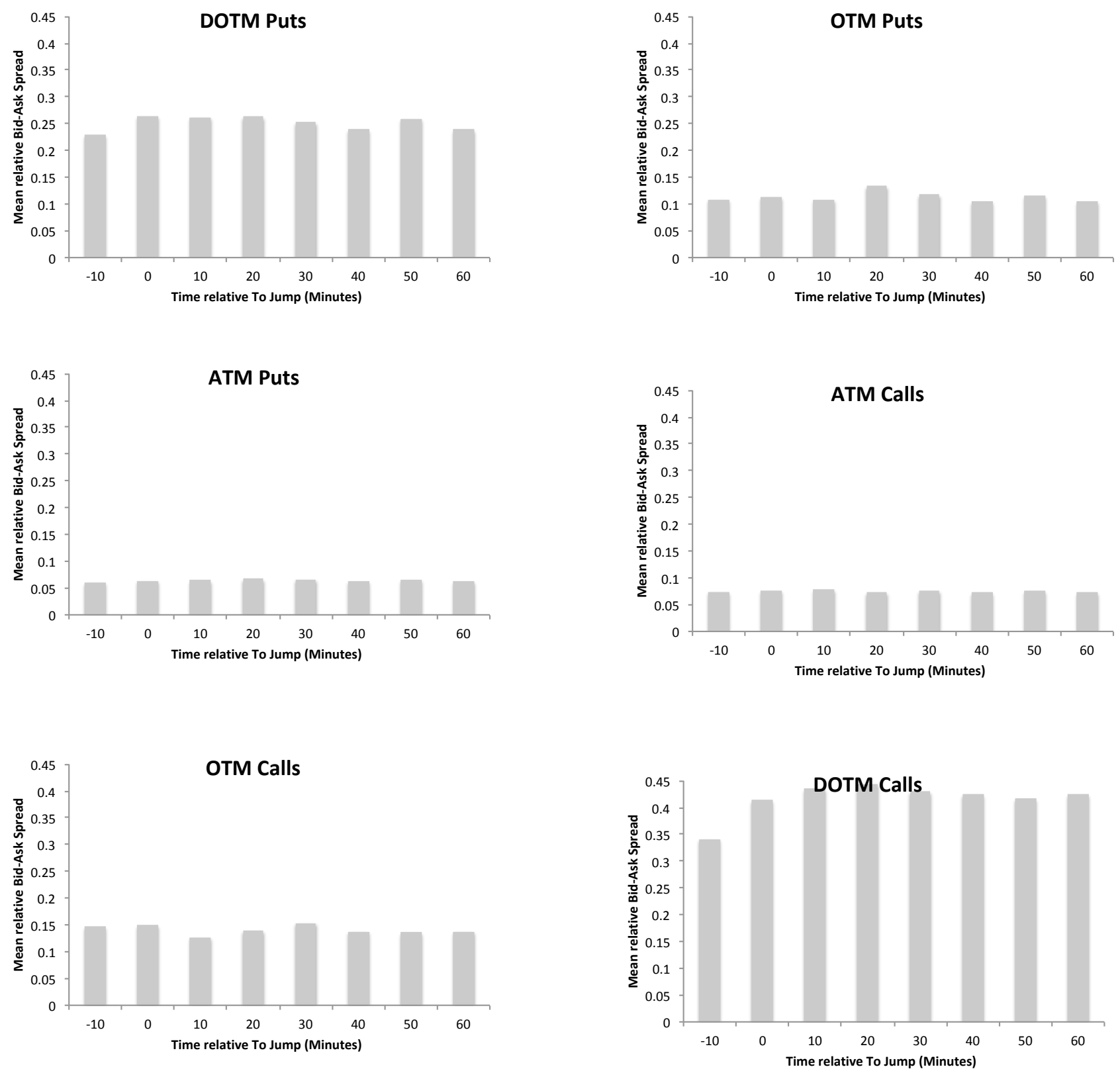
Figure OA.6: Option bid-ask spreads around jumps - S\&P 500 index options

Continued from previous page

\section{Panel C: Long-term options}
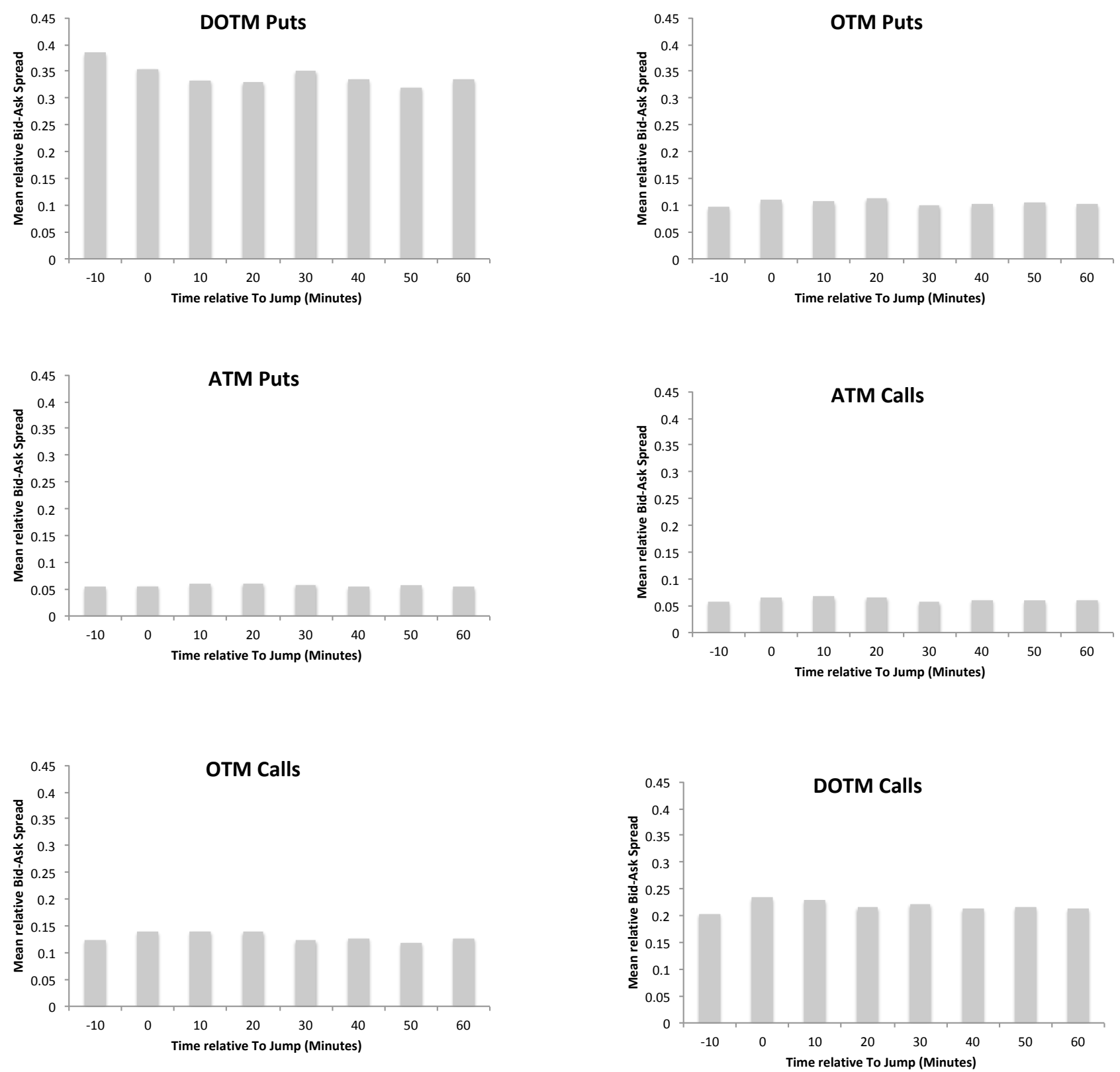

The figure shows the median relative option bid-ask spreads for a number of time subintervals around the news related jumps (10 minutes before the jump up to 60 minutes after the jump) across the various delta levels for short-, medium-, and long-term options (Panels A, B, and C, respectively). The jump time corresponds to point zero in the graph. For each maturity category, we consider deep out-of-the-money (DOTM), out-of-the-money (OTM), and at-the-money (ATM) calls and puts, as defined in Table 1 in the main paper. 